\title{
WestVirginiaUniversity
}

THE RESEARCH REPOSITORY @ WVU

Graduate Theses, Dissertations, and Problem Reports

2006

\section{Alumina waveguide characterization and SPARROW biosensor modeling}

Pavan Kumar Samudrala

West Virginia University

Follow this and additional works at: https://researchrepository.wvu.edu/etd

\section{Recommended Citation}

Samudrala, Pavan Kumar, "Alumina waveguide characterization and SPARROW biosensor modeling" (2006). Graduate Theses, Dissertations, and Problem Reports. 1786.

https://researchrepository.wvu.edu/etd/1786

This Thesis is protected by copyright and/or related rights. It has been brought to you by the The Research Repository @ WVU with permission from the rights-holder(s). You are free to use this Thesis in any way that is permitted by the copyright and related rights legislation that applies to your use. For other uses you must obtain permission from the rights-holder(s) directly, unless additional rights are indicated by a Creative Commons license in the record and/ or on the work itself. This Thesis has been accepted for inclusion in WVU Graduate Theses, Dissertations, and Problem Reports collection by an authorized administrator of The Research Repository @ WVU. For more information, please contact researchrepository@mail.wvu.edu. 


\title{
ALUMINA WAVEGUIDE CHARACTERIZATION AND SPARROW BIOSENSOR MODELING
}

\author{
By \\ Pavan Kumar Samudrala \\ Thesis Submitted to the \\ College of Engineering and Mineral Resources \\ West Virginia University \\ in partial fulfillment of the requirements for the degree of \\ Master of Science \\ in \\ Electrical Engineering \\ Committee members: \\ Dr. Lawrence A. Hornak, Committee Chairperson \\ Dr. Dimitris Korakakis \\ Dr.Aaron Timperman
}

Lane Department of Computer Science and Electrical Engineering

Morgantown, West Virginia

2006

Keywords: SPARROW Biosensor, Biolayer, Flow cell experiments, Waveguide Characterization 


\title{
Alumina Waveguide Characterization and SPARROW Biosensor Modeling
}

Pavan Kumar Samudrala

\begin{abstract}
Sensors based on evanescent wave techniques have the potential to detect minute change in refractive index arising from surface modifications. The biosensor device architecture evaluated in this work is the SPARROW (Stacked Planar Affinity Regulated Resonant Optical Waveguide) structure. This architecture can operate as a biosensor through the change in the coupled optical power resulted from antigen bonding to the bio-layer. Its stacked film arrangement with no lateral patterning offers a potentially less complex structure for fabrication. This thesis describes the process developed to optically characterize the alumina waveguides of the SPARROW device and investigates waveguide film quality as a function of fabrication parameters. Parameters include e-beam deposition drive current and oxygen flow rate. Losses of 1-3 $\mathrm{dB} / \mathrm{cm}$ have been measured for usable guiding films using the scattered power measurement technique. Microfluidic channel hybrid integration with the SPARROW device and flow cell experiments are discussed. Coupled power and coupling length variation with top waveguide thickness and index is also evaluated and discussed.
\end{abstract}




\section{Acknowledgements}

It is with pleasure that I thank many people who made this thesis possible.

I thank Dr.Hornak, my advisor and committee chair person for his valuable inputs through out the project. I also thank Dr.Korakakis and Dr. Timperman for their assistance and suggestions.

I thank Dr.Kolin Brown, my lab supervisor who had trained me with many of the lab equipment in clean room and also had been helpful to me with the lithography problems I encountered.

I would like to thank Loats Associates for this novel biosensor idea. I would also like to thank ONR for providing support for this project.

I express gratitude to fellow project members and lab colleagues for their timely help and special thanks to Sridhar Kuchibhatla for his assistance in this research.

Lastly and most importantly I would like to thank my parents whose support and suggestions had been a constant source of inspiration and motivation through out my life. My dad was particularly supportive and I have no words to thank him. 


\section{Contents}

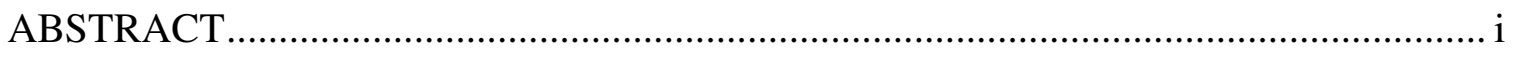

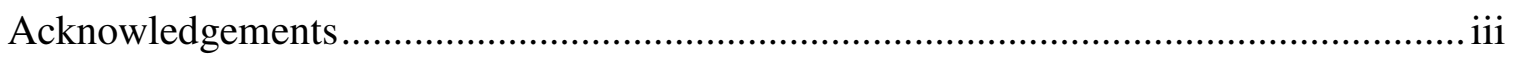

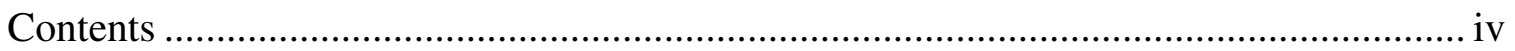

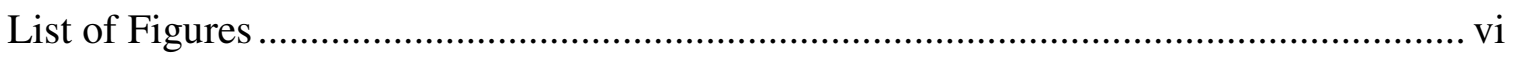

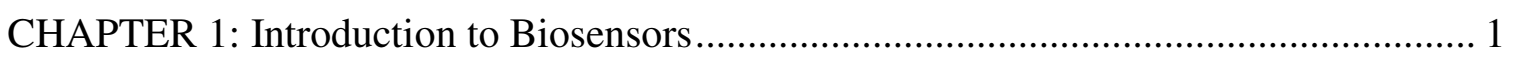

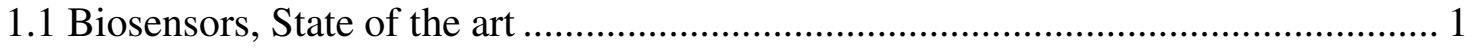

1.1.1 Biosensor Design and Applications: .......................................................... 3

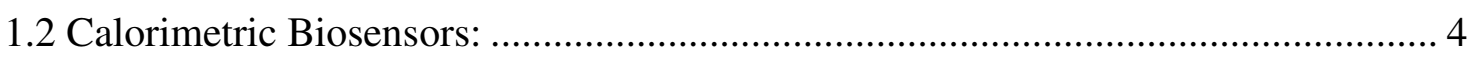

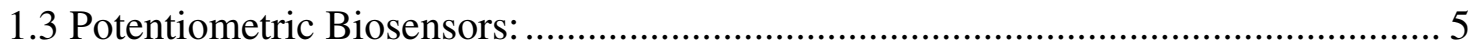

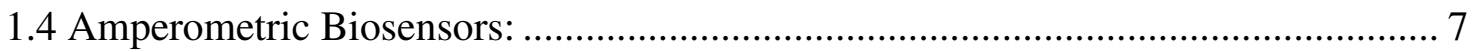

1.5 Piezo-Electric Biosensors: .............................................................................. 7

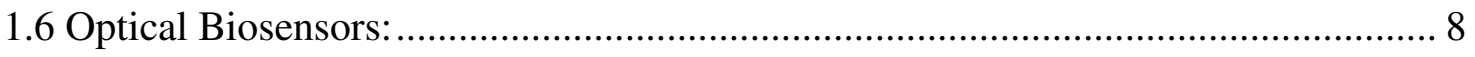

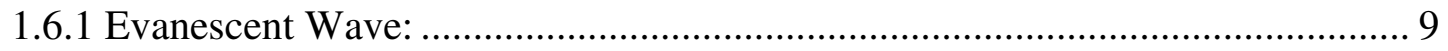

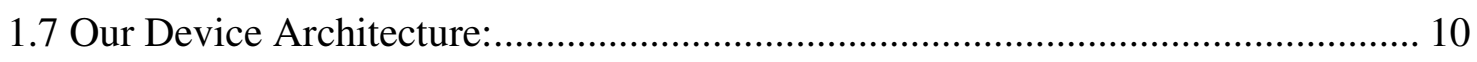

1.7.1 Operation of the SPARROW biosensor and state of art: ............................. 11

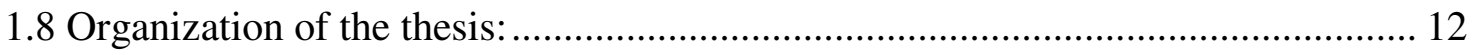

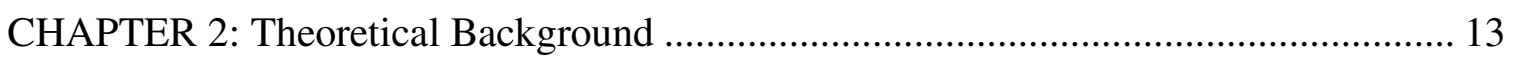

2.1 Boundary Conditions at Dielectric Interface: ................................................. 13

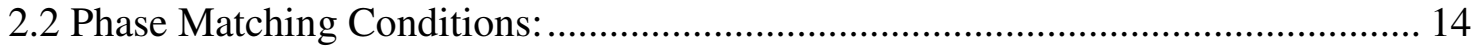

2.3 Symmetric Slab Dielectric Waveguides: ..................................................... 15

2.3.1 Analysis of Symmetric Slab Dielectric Waveguides .................................... 15

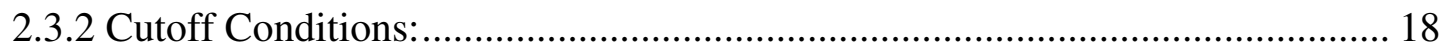

2.4 Analysis of Asymmetric Waveguides: ........................................................... 18

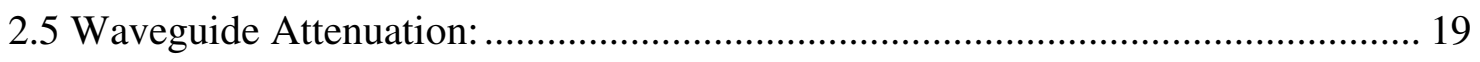

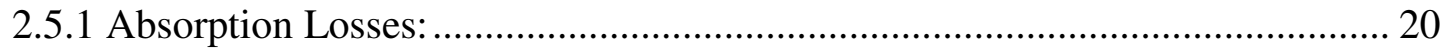

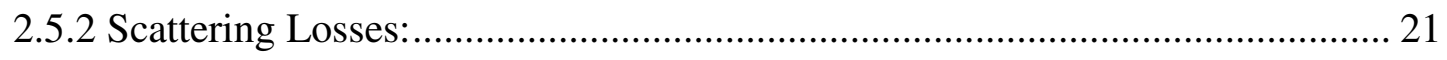

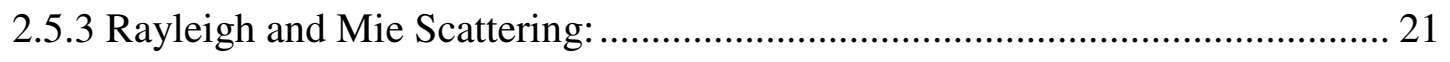

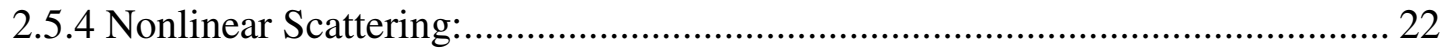

2.5.5 Radiation Losses or Bending Losses:..................................................... 22 


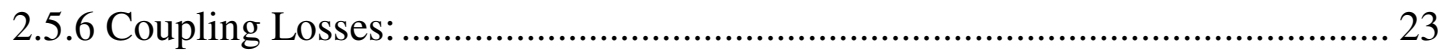

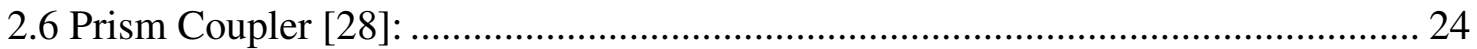

2.6.1 Frustrated Total Internal Reflection:........................................................... 24

2.6.2 Prism Coupling and Effective index [28]:………........................................... 25

2.6.3 Prism Coupler Input Firing: ........................................................................ 27

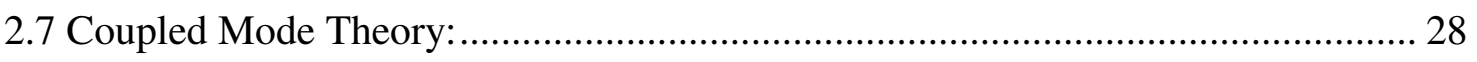

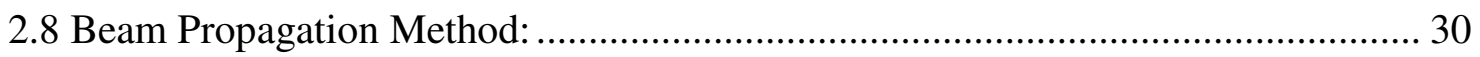

2.8.1 Slow Varying Envelope Approximation:....................................................... 31

CHAPTER 3: Modeling and Fabrication....................................................................... 33

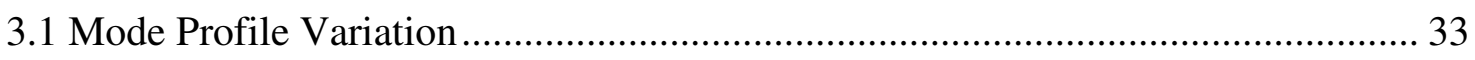

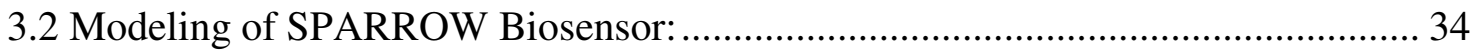

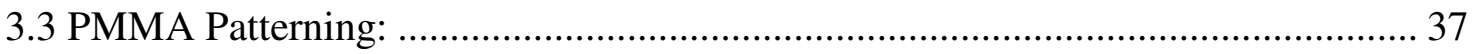

3.4 PDMS channels and Silicon Master Fabrication:.................................................. 39

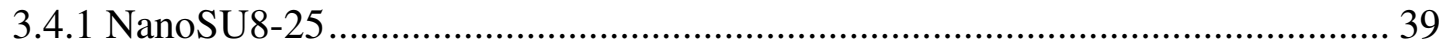

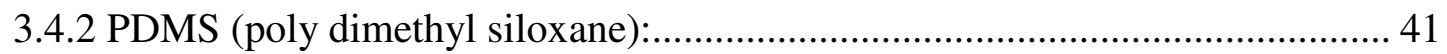

3.4.3 PDMS binding to the Alumina Surface: ........................................................ 43

CHAPTER 4: Experimental Analysis........................................................................ 45

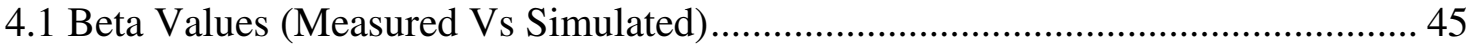

4.2 Waveguide loss variation with Ion Beam Parameters .............................................. 46

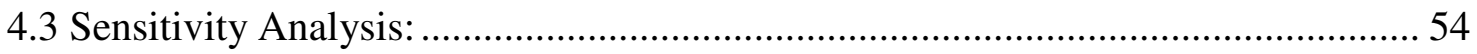

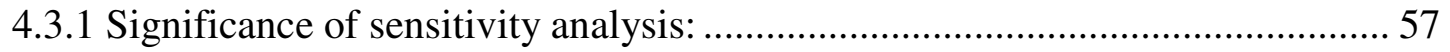

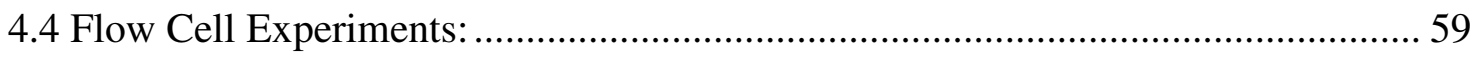

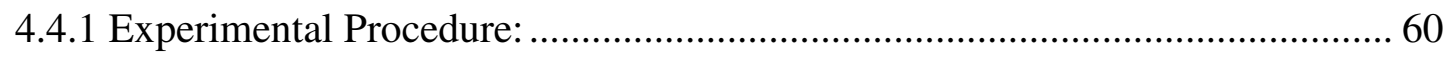

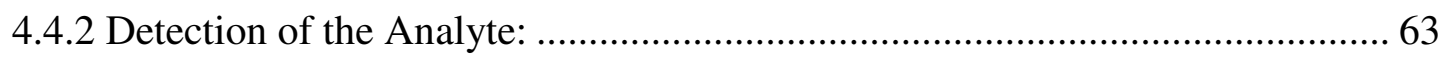

4.5 Issues with Ellipsometer Measurement/Modeling: .................................................... 66

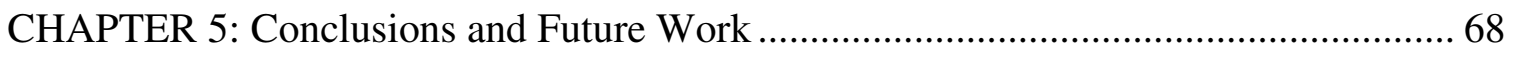

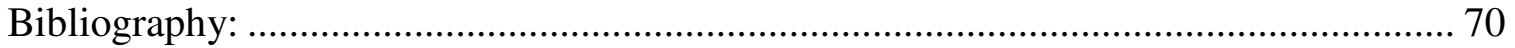

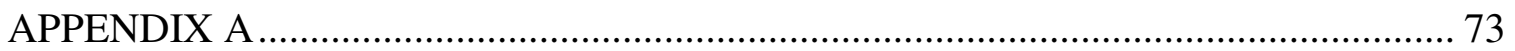

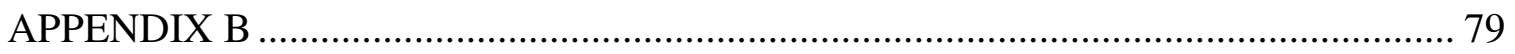




\section{List of Figures}

Fig1. 1 Schematic Diagram of Biosensor. 3

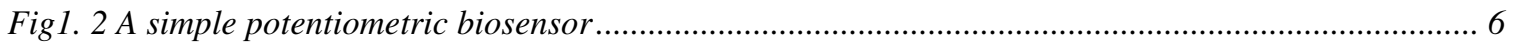

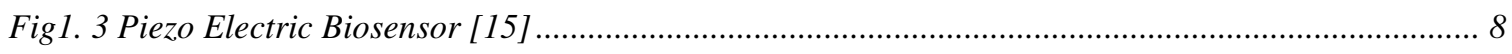

Fig1. 4 Proposed Model with Bio-layer and Microfluidc Channel .................................................... 11

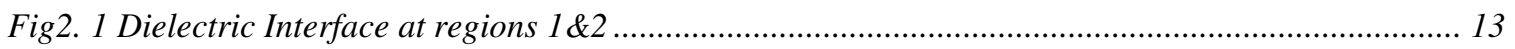

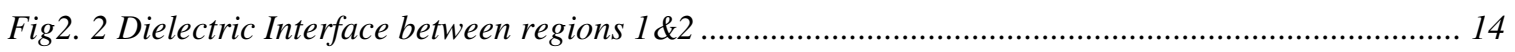

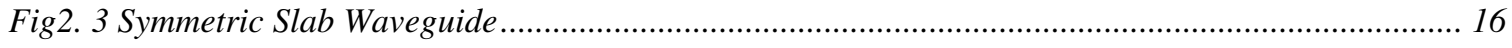

Fig2. 4 Graphical solution for guiding condition.......................................................................... 17

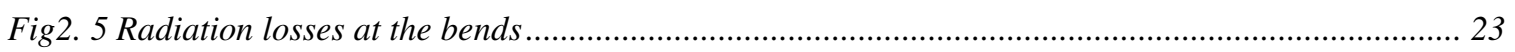

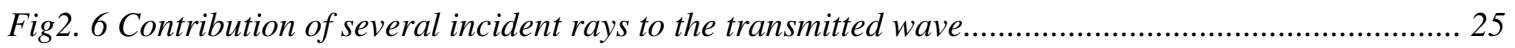

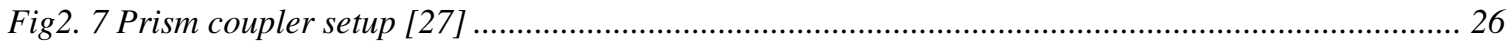

Fig2. 8 Intensity plot showing two modes of the designed structure .................................................... 27

Fig 3. 1a\&b Intensity Variation in symmetric and asymmetric waveguides. ....................................... 33

Fig 3. 2 Biosensor design and penetration depth ............................................................................ 34

Fig 3. 3a Model for simulation $\quad$ Fig 3.3b Optical Field Distribution ...................... 36

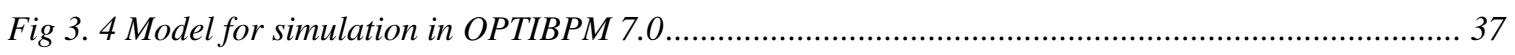

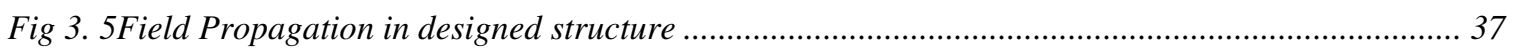

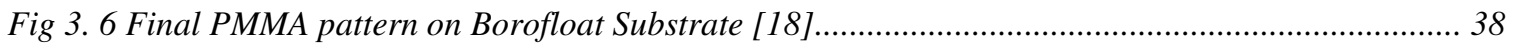

Fig 3. 7a Flow cell arrangement $\quad$ Fig 3.7b Syringe for the analyte flow............................... 39

Fig 3. 8 Spin Speed Curves for NanoSU8 [32] .............................................................................. 41

Fig 3. 9 Silicon master fabricated using Nano SU8-25 ........................................................................

Fig 3. 10 Design for PDMS mask fabrication .......................................................................... 42

Fig4. 1 Optical loss variation with ion drive current ..................................................................... 47

Fig4. 2 S Streak at $I d=1.3 A \quad$ Fig $4.2 b$ Streak at $I d=1.9$ A........................................................ 48

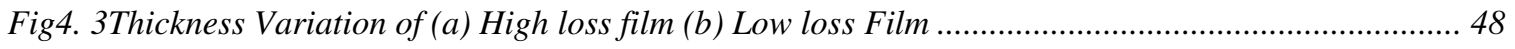

Fig4. 4 Index Variation with Drive Current .............................................................................. 50

Fig4. 5 Optical Loss Variation with Oxygen Flow rate ................................................................... 50

Fig4. 6 a $\mathrm{Id}=1.3 \mathrm{~A}$ and $\mathrm{O2}=22 \mathrm{sccm} \quad$ Fig $4.6 \mathrm{~b} \mathrm{Id}=1.3 \mathrm{~A}$ and $\mathrm{O} 2=14 \mathrm{sccm} \ldots \ldots \ldots \ldots \ldots . . . . . . . . . . .51$

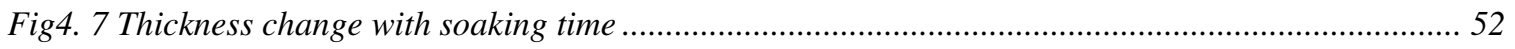

Fig4. 8 Refractive Index Variation with soaking time ............................................................. 52

Fig4. 9 Refractive index variation with annealing ........................................................................5 53

Fig4. 10 Loss variation with ion drive current and oxygen flowrate ................................................... 53 


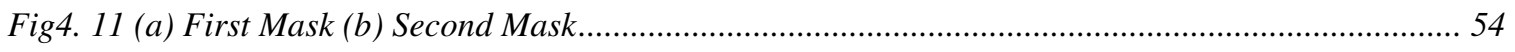

Fig4. 12(a) Power Transfer Variation with Top Waveguide Thickness and Index.................................... 55

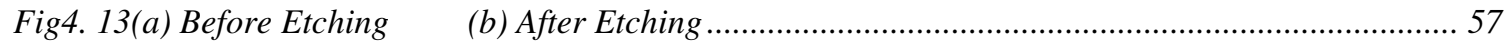

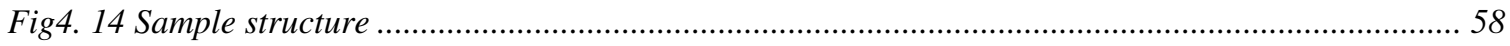

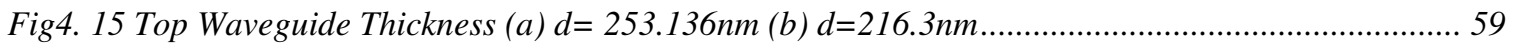

Fig4. 16 Power transfer variation between waveguides ...................................................................... 59

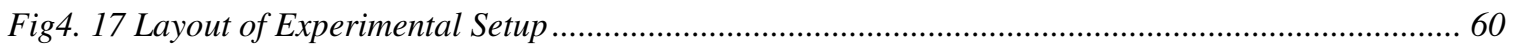

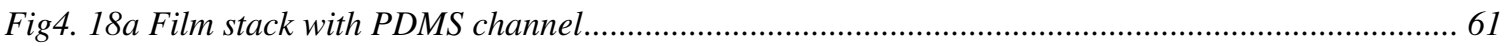

Fig4. 19 Image obtained with water in the channel ...................................................................6. 62

Fig4. 20 Loss variation in the observation area for different analytes ..................................................63

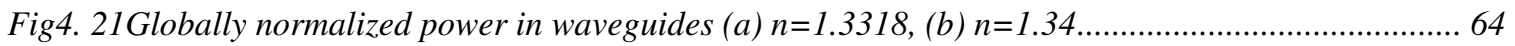

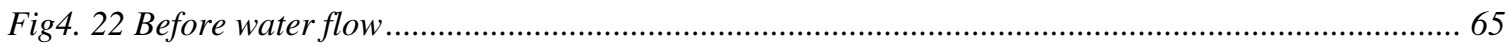

Fig4. 23Observation area a) Before water flow $\quad$ b) After water flow ............................................6 65

Fig4. 25 Index variation of the bottom waveguide after etching ......................................................... 67 


\section{CHAPTER 1: Introduction to Biosensors}

\subsection{Biosensors, State of the art}

Due to its importance in security, health, and environmental applications among others, bio-sensing is an attractive area of ongoing research. Research and development in this field is multi-disciplinary including biochemistry, physics, electrochemistry, and electronics. A biosensor is a device that detects, records and transmits information associated with the recognition of a chemical, molecule or biological material or biomolecule in the environment. Technically, a biosensor is a probe that integrates a biological component, such as a whole bacterium or a biological product (e.g., an enzyme or an antibody) with an electronic or photonic component to yield a measurable signal which is ultimately transformed into an electronic signal [1]. Biosensors have gained tremendous importance in recent decades due to their ability to continuously detect a specified substance in a mixture without perturbing the system. Further their smaller size, portability, economic viability and their insensitivity to radioactive substances make them efficient alternatives to the conventional chemical sensors [2].

Biosensors can be divided into three parts. A receptor or biolayer, transducer and detector. The biolayer is typically coupled with a transducing system which is usually optical, thermal, piezoelectric or electrochemical and is selectively sensitive to the analyte to be detected. The first biosensor was an enzyme based glucose amperometric sensor developed by Clark and Lyons in 1962 based on $\mathrm{pH}, \mathrm{pCO}_{2}$ and $\mathrm{pO}_{2}$ [3]. Since then many biosensors have been developed based on different techniques.

Sensors types can be divided into physical, chemical and biochemical. The physical sensors are used to measure parameters such as the temperature and pressure and are typically not meant for particle detection. A chemical sensor is typically a compact miniaturized device that transforms chemical information, such as variation of concentration, into a physically measurable signal. Specifically the selective and reversible detection is accompanied by a concentration dependent electrical signal [4]. 
Various electronic signals are employed in order to observe the variation in the parameters such as current, electrical voltage, capacitance, and impedance.

Biosensors are special sensors that use a biochemical mechanism for their recognition system. The bio-recognition system usually consists of [Fig 1.1] receptors like proteins, enzymes, microorganisms or antibodies that translate the information from biochemical domain to a chemical or physical output signal. As the bio-recognition systems are highly selective compared to inorganic catalysts, these types of sensors are the widely used. However, biosensors have a few limitations. The most important of them is the instability of the receptor molecules and their gradual degradation with time [4]. Hence increasing the stability of the bio-molecule is one of the major limitations of a biosensor. This can be achieved by using immobilization process where organic chains selective to the bio-agents are grown on the probe surface as well as employment of an architecture that enable controlled regeneration of the sensor surface [5].

The evolution of the field of biosensors can be divided into three generations. The first generation biosensors are based on the products formed after the reaction between the analyte and the enzymes (biolayer). The products thus formed reach the transducer and produce a signal, usually electric. The first biosensor proposed by Clark and Lyons and developed further by Updike and Hicks is of this type. The operation principle of this sensor is as follows. Glucose oxidase is immobilized on the platinum electrode and is followed by the liberation of hydrogen peroxide along with electrons and the signal is measured amperometrically at the electrode surface [3]. The second-generation biosensors do not depend on the products of the reaction but they adopt agents or mediators between reaction and transducer. The first 'second generation biosensor' was also a glucose biosensor developed by the company Medisense and they used artificial electron mediators [6]. The third generation biosensors do not involve any mediators as the reaction itself causes the response. This type of sensors have all the advantages of the second generation with added features like self contained nature, continuous monitoring, less cost and repeated measurements. TheraSense is one of many companies working on this kind of sensors currently at a product development level [6]. 
The following are few important characteristics that biosensors must possess. [7].

1) Biosensors can be as specific as the immobilized system used in them but should be stable under normal storage conditions and show good stability over a large number of assays.

2) The dependence on variations of parameters like temperature, stirring, electromagnetic interference, etc should be negligible as that would allow minimal pretreatment for analysis and make it robust to general atmospheric conditions.

3) The biosensor should be low priced, small, portable and capable of being used by semiskilled people.

\subsubsection{Biosensor Design and Applications:}

Fig 1.1 shows the schematic diagram of the biosensor. It can be seen that the biosensor can be divided mainly into two regions the receptor and the transducer. Receptor has a layer of antibodies or any other bioagent sensitive material usually called bio-layer which would produce a characteristic change in a parameter which is transmitted as a signal to the detector by a transducer.

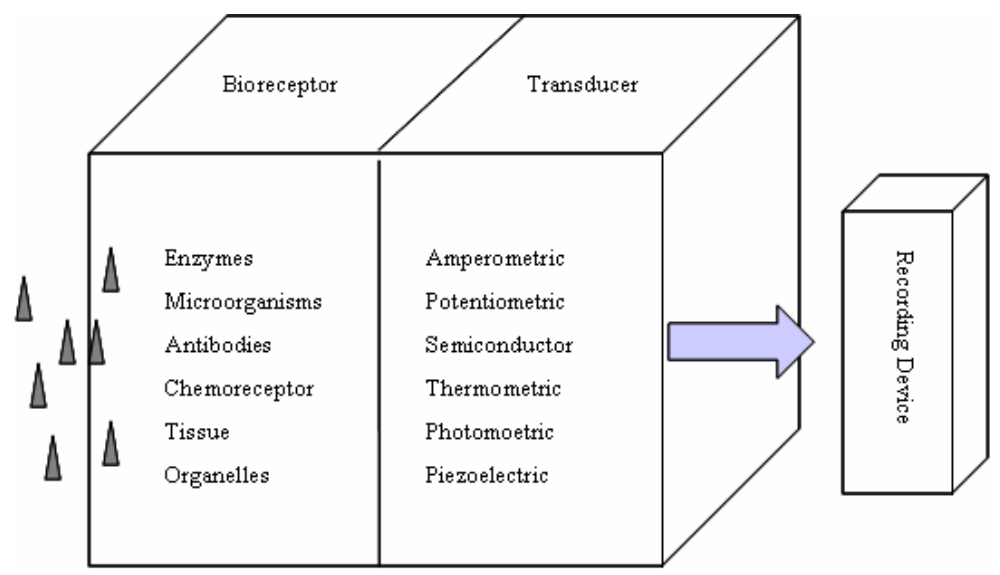

Fig1. 1 Schematic Diagram of Biosensor 
The transducer forms an integral part of the biosensor. Transducers are sensors that can detect or measure the presence of various chemicals and produce a significant change in their output signal. A transducer makes use of the physical change that accompanies the biological reaction. The change can be one of the following [9].

- The heat output or absorption produced by the biological reaction (calorimetric biosensors)

- Change in the local area charge thus causing an electric voltage.(Potentiometric Biosensors)

- Motion of the electrons due to reaction. (Amperometric Biosensors)

- Change in Power Output or Absorption. (Optical Biosensors)

- Changes produced due to the mass of the bioagents. (Piezo-Electric Biosensors)

Biosensors have numerous applications ranging from home diagnostics to security applications. Apart from home diagnostics, medical and security applications have immense potential for biosensors. Biosensors not only are useful in detecting biological threats like anthrax but also are extremely important components of numerous nonbiological applications which sense toxic agents and thus avert illness. Defective or infected cells can be detected effectively using biosensors. SDBS (super Dimension Bronchus system) is one such device which is used to detect suspicious masses in the lungs [10]. Specific biosensors have already been developed for detecting diseases like Avian Influenza (commonly knows as Bird Flu), glucose sensor [11] and research towards detecting dangerous diseases like cancer [12] is in progress. Food sector, environmental monitoring, blood glucose detection etc are some of the sectors already exploited by the biosensors. The newest application which is gaining prominence is DNA detection [13].

Next we would overview each basic biosensor transduction mechanism.

\subsection{Calorimetric Biosensors:}

Calorimetric biosensors operate on temperature change to detect the bio-agent. The heat liberated from reaction between enzyme and analyte is used to measure the rate of 
reaction and thus the concentration of analyte. Thermistors are normally used to detect these temperature changes as they change their electrical resistance with temperature. The equation that relates them is [9]

$$
\ln \left(\frac{R_{1}}{R_{2}}\right)=B\left(\frac{1}{T_{1}}-\frac{1}{T_{2}}\right)
$$

where $R_{1}$ and $R_{2}$ are the resistances of thermistors at temperatures $T_{1}$ and $T_{2}$ respectively. Assuming $T_{1} \approx T_{2}=T_{1}$ the above equation turns out to

$$
\frac{\Delta R}{R}=-\left(\frac{B}{T_{1}^{2}}\right) \Delta T
$$

We observe that heat produced in the reaction changes the resistance which is converted to an electrical signal using balanced Wheatstone bridge. A major hurdle with this type of biosensor is to obtain a reference and measurement thermistor with same characteristic temperature constants. This is an example for a physical sensor coupled with a bio-layer for bio-sensing.

\subsection{Potentiometric Biosensors:}

Potentiometric biosensors have indicating electrodes, reference electrodes and a reagent on the indicating electrode. Fig 1.2 shows the schematic diagram of potentiometric biosensor. The transducer mainly consists of a thin immobilized enzyme membrane on which the reaction takes place. A potential is generated at the membrane based on the concentration of cations. The reaction is monitored by observing the potential at the indicating electrode surface. The potentiometric biosensor measures the change in the potential at the indicating electrode surface after the fluid sample containing the analyte is added to the reagent. The change in potential that occurs is correlated to the detection or measurement of analyte in the fluid sample.

There are two types of electrodes that are used in potentiometric biosensors. Ion selective electrode develops electrical potential proportional to the logarithm of the 
activity of an ion in the solution. These electrodes respond truly to one specific ion. The response of the ion selective electrode is given by [8]

$$
E=E_{0}+(2.303 R T /(z F)) * \log (A)
$$

where $\mathrm{E}$ is the measured potential, $\mathrm{R}$ is the gas constant, $\mathrm{T}$ is the absolute temperature, $\mathrm{F}$ is the Faraday constant, $\mathrm{E}_{0}$ is the characteristic constant of Ion Selective Electrode and A is the ion activity.

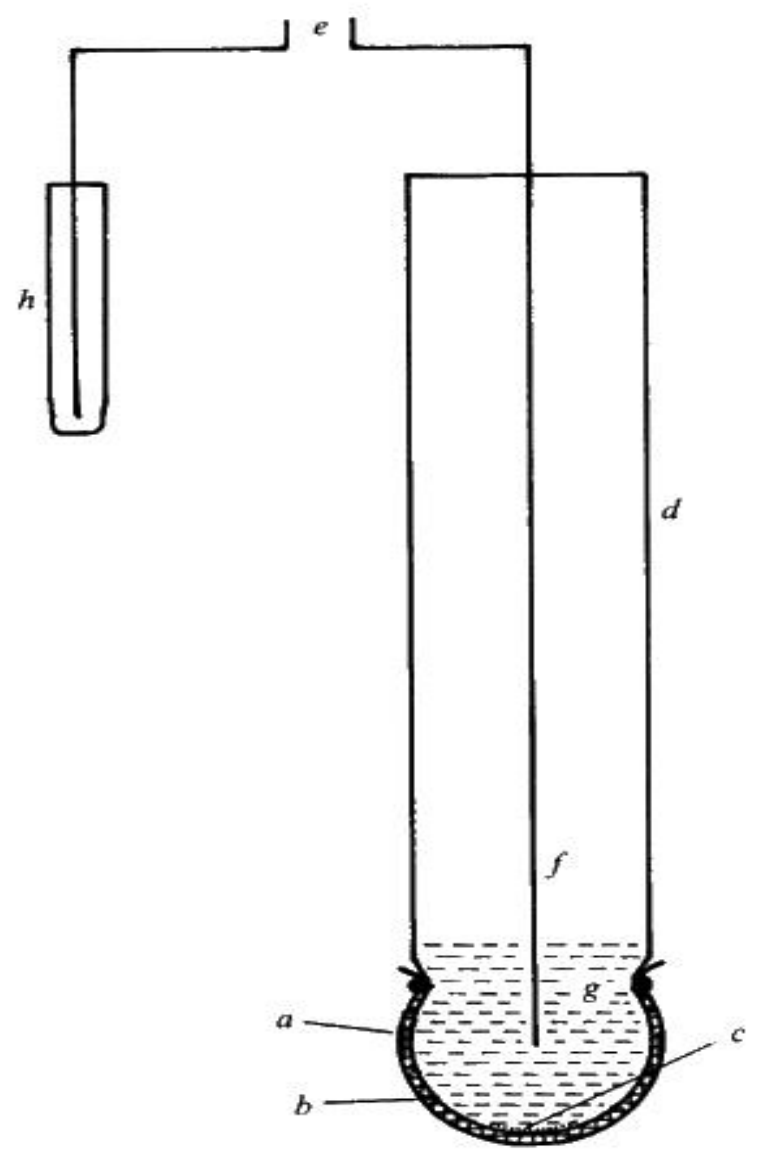

Fig1. 2 A simple potentiometric biosensor

Fig 1.2 shows a general potentiometric biosensor where ' $\mathrm{a}$ ' is semi permeable membrane, ' $b$ ' is biocatalyst, ' $c$ ' is active glass membrane, ' $d$ ' is $\mathrm{pH}$ probe, ' $e$ ' is the electrical potential, ' $f$ ' is the electrode, ' $g$ ' is the solute and ' $h$ ' is the reference electrode.[9] 


\subsection{Amperometric Biosensors:}

Amperometric sensing is based on measuring the current produced by oxidation or reduction of an electro-active compound at a working electrode/ sensor. A constant potential is applied to the working electrode and this potential acts as an electrochemical driving force and results in oxidation or reduction of the compound to be measured. Provided concentration of the reactant remains the same, the reaction produces a stable potential. By applying Faradays law, current can be defined as [6, 14]

$$
I=n F(d a / d t)
$$

Where the current (I), represents the electrochemical oxidation/reduction rate of the analyte at the electrode. $d a / d t$ is the rate of change of analyte concentration, $F$ is the Faradays constant and $n$ is the number of electrons transferred in the reaction. The reaction rate depends on electron transfer and analyte mass transport. The rate of reaction can be increased by increasing the applied potential. At some point the potential saturates and the rate is limited only by the mass transport of the reactant to the electrode. If the reaction is very fast then the concentration of analyte at the electrode is zero, and the maximum overall rate of reaction is reached. At this point the current can be given by the following expression.

$$
I=\gamma d F D\left(\frac{d C}{d X}\right)_{x=0}
$$

Here $d C / d X$ is the rate of change of concentration of electro-active species to the electrode surface. ' $A$ ' is the electrode area and ' $D$ ' is the diffusion coefficient. The rate of mass transport to the electrode depends on the bulk concentration of analyte, the electrode shape and area, and diffusion and convection conditions. The first biosensor developed by Clark and Llyons was an amperometric biosensor and is based on the reduction of oxygen. [3]

\subsection{Piezo-Electric Biosensors:}

Piezo-electric crystals vibrate when they are placed in electric field. The frequency of oscillation depends on the mass of the crystal, thickness and cut. This 
frequency changes due to the absorption or desorbtion of mass from the surface of the crystal. The variation in the frequency is given by [15]

$$
\Delta f=K f^{2} \Delta m / A
$$

Where $\Delta f$ is the change in the resonant frequency, $\Delta m$ is the change in the mass of adsorbed material, $K$ is constant that depends on the material properties and physical structure of the crystal and ' $A$ ' is the surface area of the crystal surface. For any piezoelectric crystal the change in frequency is proportional to the mass of the crystal upto about $2 \%$ of change [9]. Frequency change produced by change in mass on the crystal surface is used to detect bio-agent. Hence a highly sensitive chemical or physical binding that selectively binds to the particles to be detected has to be coated for sensing applications.

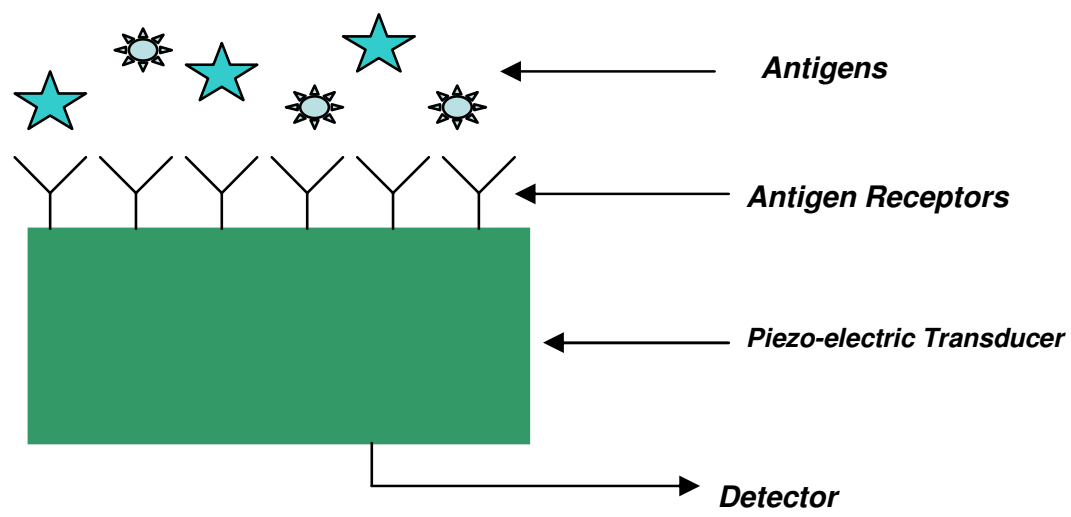

Fig1. 3 Piezo Electric Biosensor [15]

\subsection{Optical Biosensors:}

Optical biosensors are significant interest due their potential sensitivity and portability. Typical optical biosensor consists of a source of light (usually a laser or an LED designed to operate at a particular wavelength), tools to input light in a proper shape to some modulating agent, and then a detector. Optical biosensors are presently primarily of three types. Evanescent wave based, surface plasmon resonance based and photonic crystal based sensors. Surface Plasmon sensors are based on the evanescent waves produced at the interfaces due to the electron excitation. Photonic crystal sensors are based on the localization of the power at certain frequencies when light is passed through a photonic crystal. Evanescent wave based sensors employ waveguides and are based on 
the interaction of evanescent wave with that of the bioagent and the resultant change in a wave parameter is used to detect the presence of bio-agents. Of these three techniques surface plasmon resonance based sensors are the most commercially mature but tend to be expensive and large and hence may not be useful for real time applications while evanescent and photonic crystal based sensors hold significant potential for integration and are the subject of intense investigation. Evanescent sensors are very sensitive to the surface and hence a layer, usually organic, is grown on the surface which selectively traps the bioagents and produces a change in its optical characteristics. Photonic crystal approaches to date tend to draw upon changes in transmission properties in the crystal upon similar changes in material optical characteristics upon binding within selected regions of the crystal lattice [16]. Here we focus on evanescent wave detection as a means to achieve biosensor integration.

\subsubsection{Evanescent Wave:}

Evanescent waves are electromagnetic waves that propagate along the direction of light and decay exponentially with distance perpendicular to it. The reflection of sinusoidal waves with incidence angle greater than critical angle at an interface produces evanescent waves due to total internal reflection [17]. They arise when light travel from higher index medium to a lower index material.

$$
E=E_{o} \exp \left(-x / d_{p}\right)
$$

where ' $d_{p}$ ' is called the 'penetration depth' and is equal to the distance ' $\mathrm{x}$ ' at which the intensity of the wave reduces to $1 / e$ of the original intensity.

Evanescent waves are very sensitive to the surface and any change on the surface would result in change of the phase of the propagating wave. This property can be used in bio-sensing applications. Evanescent waves can interact with the molecular species if present in the 'penetration depth' range and this would effect the propagation of light. In reality, the effect of the antigens bonded with antibodies immobilized on the surface of a metal or dielectric surface change the effective refractive index of the layer in its vicinity. Hence an evanescent wave sees a different refractive index which brings a 
phase shift in the propagating wave. The following are the advantages of the evanescent sensors

- Considerable miniaturization is possible

- Enhanced sensitivity over conventional optical methods because of the greater power present in the evanescent field.

\subsection{Our Device Architecture:}

The sensor we chose to explore is an evanescent sensor which works on the SPARROW (Stacked Planar Affinity Regulated Resonant Optical Waveguide) technique [5]. It has two single mode resonant coupled alumina waveguides stacked vertically with a spacer of silica on a glass substrate as shown in the Fig 1.4. A resonant coupled waveguide biosensor design has been chosen for its higher sensitivity and simple stacked structure. The light is coupled onto the bottom waveguide and is observed in the bottom waveguide using a CCD camera. The bioagent to be detected is flown through the 'biolayer grown microfluidic channel' which specifically absorbs the bioagents to be detected. The device is designed in such a way that light can be observed in the bottom waveguide only at a specific refractive index material of the analyte.

\begin{tabular}{|llll|}
\hline Alumina & $\mathrm{n}=1.659$ & $\mathrm{~d}=194 \mathrm{~nm}$ & Waveguide \\
\hline Silica & $\mathrm{n}=1.457$ & $\mathrm{~d}=1100 \mathrm{~nm}$ & Spacer \\
\hline Alumina & $\mathrm{n}=1.659$ & $\mathrm{~d}=144 \mathrm{~nm}$ & Waveguide \\
\hline Borofloat & $\mathrm{n}=1.4701$ & Substrate \\
\hline
\end{tabular}

Table 1.1 Details of the Materials used in the Biosensor Design

The materials used in this device have to be very stable in an aqueous environment as the device operates in a liquid environment. Hence films of alumina and silicon dioxide were chosen for their stable refractive index and thickness at ambient conditions [18]. Microfluidic channels have been fabricated using a polymer (PDMS) and then bonded to the alumina surface using oxygen plasma etching and hydrolyzing the surface. The waveguides should obey the resonance condition for the device operation which means that power from one waveguide should transfer into the other waveguide 
when the desired refractive index is present in the channel. Table 1.1 shows a model of a SPARROW sensor where light from one waveguide is transferred completely to other waveguide when the analyte index is 1.3318. Based on the index of bioagent bound on the waveguide surface the power coupled into the bottom waveguide changes.

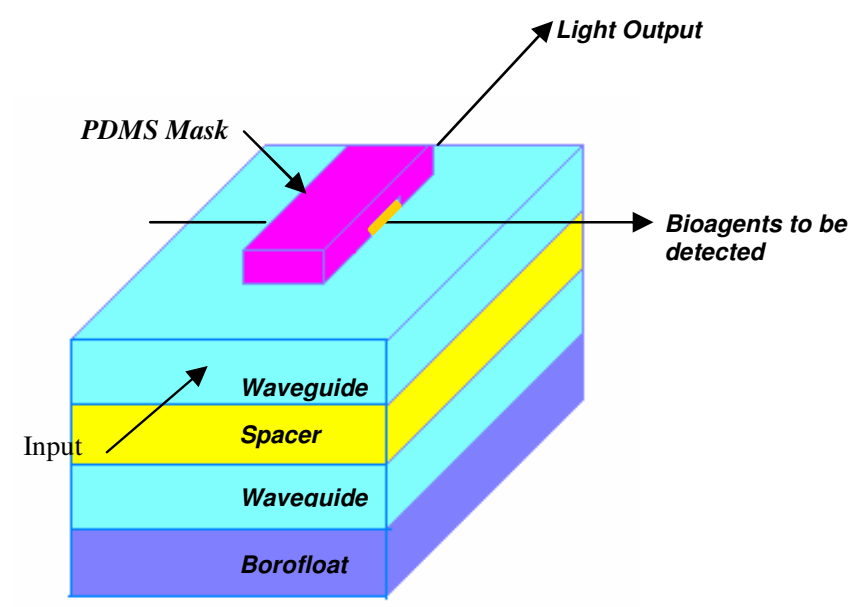

Fig1. 4 Proposed Model with Bio-layer and Microfluidc Channel

\subsubsection{Operation of the SPARROW biosensor and state of art:}

SPARROW biosensors work on the principle of resonance between the waveguides. It has a biolayer which acts as a receptor (Fig 1.1), a stacked coupled waveguides (Fig 1.4) that acts as a transducer and a CCD camera is the recording device or the detector. In ideal operation of the device, there should be no power detected from the top waveguide when there are no bio-agents bonded on the biolayer. Presence of bioagents alters the effective refractive index of the biolayer and thus produces a change in the power detected at the ends of the waveguide. The change is power is correlated to the refractive index and thus the index of the bio-agent is determined.

The major issues of this sensor are designing and fabrication of coupled waveguides, bio-layer design, wave guide characterization and flow cell experiments. Fabrication of coupled waveguides as per the design is very important as a single nanometer change from the design would produce a lot of variation in the power at the ends of the waveguide. The biolayer designed should be less in height as the sensitivity of the biosensor is within the penetration depth of the evanescent wave. This is usually 
around $100 \mathrm{~nm}$ and hence the biolayer height should be as less as possible. Power observed at the ends of the waveguide is dependent on attenuation present in the wave guide. Hence the waveguides have to be optically characterized to determine the actual power observed at the ends of the waveguide. The final issue is with the flow cell experiments. The employed setup should be such that there is no change in the coupling spot before and after the bio-agent bonding. This means that the whole setup meant for flow cell experiments has to be physically at the same location before and after the bioagent bonding.

This thesis aims at designing resonant coupled waveguides, performing waveguide characterization and setting up for flow cell experiments. Sensitivity analysis was also made to see the variation produced in power and coupling length due to change in thickness and refractive index of the top wave guide.

\subsection{Organization of the thesis:}

The outline of this thesis is as follows. Chapter 2 discusses the basic attenuation principles, light propagation in optical waveguides, prism coupling and beam propagation method for optical simulations which aid in understanding the experimental work done. Chapter 3 discusses the computer simulations of the designed structure along with the limitations the tool has. I would also discuss the fabrication details which include PMMA patterning, silicon master fabrication for making PDMS molds and bonding of PDMS molds onto the alumina waveguide. Chapter 4 discusses the method employed for characterizing the waveguides using scattered power measurement technique adopting

prism coupler and a CCD camera. Chapter 5 details the experimental results with the beta values, waveguide loss variation with ion beam parameters like oxygen flow rate and ion drive current, sensitivity analysis and transducer measurements. 


\section{CHAPTER 2: Theoretical Background}

It is important to understand the concepts of wave propagation of light and its behavior when propagating in mediums like waveguides. Ray optics though can explain many of the phenomena, wave optics is more generalized form of light propagation. Propagation of light in a waveguide and changes caused by another waveguide in its vicinity are discussed here using wave theory. The device design has two resonant coupled waveguides. The mechanism of power transfer between them and the effect of index of refraction of the waveguides on the light propagation is also discussed.

\subsection{Boundary Conditions at Dielectric Interface:}

Electromagnetic wave behavior at the interfaces can be well understood from the boundary conditions. From Maxwell's equations [20]

$$
\begin{aligned}
& \oint_{c} E . l d l=-\frac{\partial}{\partial t} \oiint_{s} B . n d S \\
& \oint_{c} H . l d l=\frac{\partial}{\partial t} \oiint_{s} D . n d S
\end{aligned}
$$

Where $c$ bounds the surface $S$ and $\stackrel{\Lambda}{n}$ is the normal unit vector to the differential elemental area $d S$ as shown in Fig.2.1. As $\Delta \mathrm{h}$ approaches zero while $\Delta \mathrm{l}$ is kept small $\mathrm{B}$ and $\mathrm{D}$ become finite. Also the R.H.S. of the equations (2.1 \& 2.2) approaches zero due to the reduction in area. Hence the above equations approach to

$$
\begin{aligned}
& \left(E_{\tan }\right)_{1} \Delta l-\left(E_{\tan }\right)_{2} \Delta l=0 \\
& \left(H_{\tan }\right)_{1} \Delta l-\left(H_{\tan }\right)_{2} \Delta l=0
\end{aligned}
$$

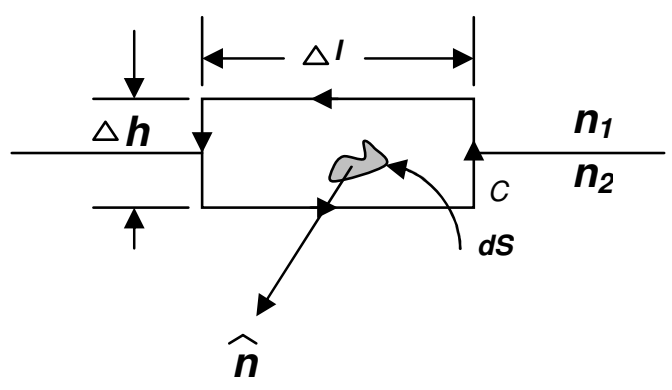

Fig2. 1 Dielectric Interface at regions $1 \& 2$ 
From the above equations we observe that the tangential electric and magnetic field components should be continuous at the interfaces.

\subsection{Phase Matching Conditions:}

Let the incident, reflected and transmitted plane waves have propagation constants of $k_{i}, k_{r}$ and $k_{t}$ respectively. Then the corresponding waves can be written as

$$
\begin{aligned}
& E_{i}(r)=A_{i} e^{-j\left(k_{i} \cdot r\right)} \\
& E_{r}(r)=A_{r} e^{-j\left(k_{r} \cdot r\right)} \\
& E_{t}(r)=A_{t} e^{-j\left(k_{t} \cdot r\right)}
\end{aligned}
$$

According to $\mathrm{Eq}(2.3)$ the tangential electric fields should be continuous across the boundary. Thus we get

$$
E_{i}(0, y, z)+E_{r}(0, y, z)=E_{t}(0, y, z)
$$

The only nontrivial solution for the above solution is that $k_{i y}=k_{r y}=k_{t y}$ and $k_{i z}=k_{r z}=k_{t z}$. The following equations infer that the propagation constants along the tangential direction are maintained constant at interfaces. In Fig 2.2 the propagation constants remain same along $y$ and $z$ directions. These relations are known as phase matching relations. [20]

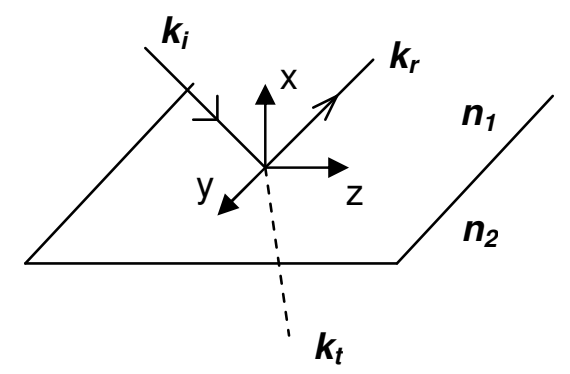

Fig2. 2 Dielectric Interface between regions $1 \& 2$ 


\subsection{Symmetric Slab Dielectric Waveguides:}

Symmetrical slabs are the simplest waveguide geometry. Consider a slab dielectric waveguide as shown in Fig 2.3. For simplicity we assume that the waveguide is homogeneous along the $y$ direction and very long compared to the wavelength of light. Hence working in the $x-z$ plane should be sufficient as the axes can be interchangeably rotated. Let the input wave be a plane wave, then Electric field, E and magnetic field, $\mathrm{H}$ can be represented as [20]

$$
E(x, y, z)=E(x, y) e^{-j k_{z} z}, \quad H(x, y, z)=H(x, y) e^{-j k_{z} z}
$$

where $k_{z}$ is the propagation constant.

The reflections at each interface are also dependent on the polarization of the input wave and hence we would confine our analysis to TE waves only. The wave equation in a source free homogenous medium is given by [21]

$$
\nabla^{2} E+k^{2} n^{2} E=0
$$

\subsubsection{Analysis of Symmetric Slab Dielectric Waveguides}

Let us assume that the waveguide extends to infinity (usually ten wavelengths) along the $y$ direction. Since it is symmetric waveguide geometry we have the same refractive indices on both sides of the waveguide. Also wave equation in each region can be represented as [22]

$$
\begin{array}{ll}
\frac{\partial^{2} E(x, y)}{\partial x^{2}}+\left(k^{2} n_{1}{ }^{2}-k_{z}{ }^{2}\right) E(x, y)=0 & \text { Cladding } \\
\frac{\partial^{2} E(x, y)}{\partial x^{2}}+\left(k^{2} n_{2}{ }^{2}-k_{z}{ }^{2}\right) E(x, y)=0 & \text { Waveguide }
\end{array}
$$




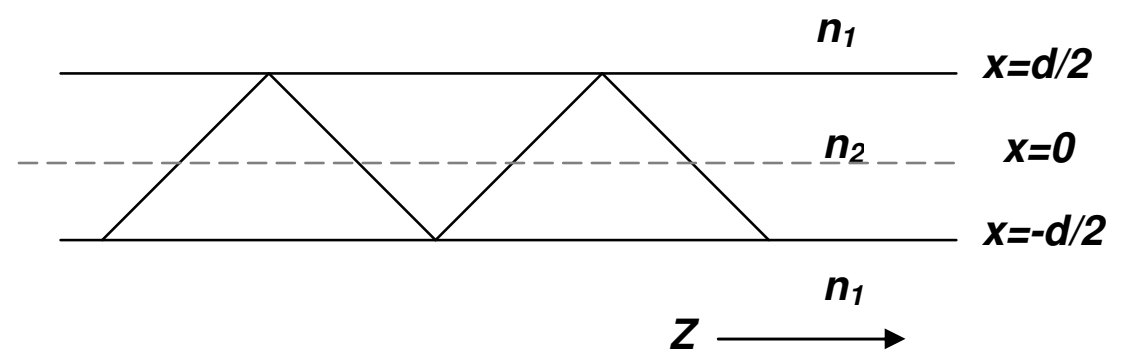

Fig2. 3 Symmetric Slab Waveguide

The solutions to the above equations describe the propagation of light in both the mediums and are called modes. A mode is a spatial distribution of optical energy in one or more dimensions [22]. A guided mode is a set of electromagnetic fields which maintain their transverse spatial distribution along the propagating direction. A guided wave consists of a non planar wave decaying in $\pm x$ direction in the cladding region while maintaining a propagation constant of $k_{z}$ in the $z$ direction. The electric field component in these regions is

$$
\begin{array}{rlrc}
E_{y}(x) & =C_{1} \exp \left(-\alpha_{1} x\right) & \mathrm{x}>\mathrm{d} / 2 & \text { Cladding } \\
& =C_{2} \cos \left(k_{2 x} x\right) & -\mathrm{d} / 2<\mathrm{x}<\mathrm{d} / 2 & \text { Waveguide region } \\
& =C_{1} \exp \left(\alpha_{1} x\right) & \mathrm{x}<\mathrm{d} / 2 & \text { Substrate }
\end{array}
$$

Where $\alpha_{1}=\sqrt{k_{z}^{2}-\omega^{2} \mu \varepsilon_{1}}$ and $k_{2 x}=\sqrt{\omega^{2} \mu \varepsilon_{2}-k_{z}^{2}}$.

$\mathrm{C}_{1}$ is used on both sides of the waveguide due to symmetry and this would change if the refractive indices on the two sides of the waveguide are different. A cosine wave in the waveguide region would give us even modes while sine wave would give us odd modes. We will discuss even modes first. Similar to electric field, magnetic field components can be written using Maxwell's curl equation. [20]

$$
H_{z}(x, z)=\frac{j}{\omega \mu} \frac{\partial}{\partial x} E_{y}(x, z)
$$

Applying the above equation we get

$$
\begin{aligned}
H_{z}(x) & =-\frac{j}{\omega \mu} \alpha_{1} C_{1} \exp \left(-\alpha_{1} x\right) & & \mathrm{x}>\mathrm{d} / 2 \\
& =-\frac{j}{\omega \mu} k_{2 x} C_{2} \sin \left(k_{2 x} x\right) & & -\mathrm{d} / 2<\mathrm{x}<\mathrm{d} / 2
\end{aligned}
$$




$$
=\frac{j}{\omega \mu} \alpha_{1} C_{1} \exp \left(\alpha_{1} x\right) \quad \mathrm{x}<\mathrm{d} / 2
$$

Applying boundary conditions at the waveguide surface $(x=\mathrm{d} / 2)$ we get

$$
C_{1} \exp \left(-\alpha_{1} d / 2\right)=C_{2} \cos \left(k_{2 x} d / 2\right)
$$

Similarly for magnetic field at $x=-\mathrm{d} / 2$, we get

$$
\alpha_{1} C_{1} \exp \left(-\alpha_{1} d / 2\right)=C_{2} k_{2 x} \sin \left(k_{2 x} d / 2\right)
$$

Solving Eq $2.13 \& 2.14$ we get

$$
\tan \left(k_{2 x} d / 2\right)=\alpha_{1} / k_{2 x}
$$

Eq 2.15 is known as the guidance condition and its solutions are known as even modes. Fig 2.4 shows the graphical solution for even modes of a slab waveguide.

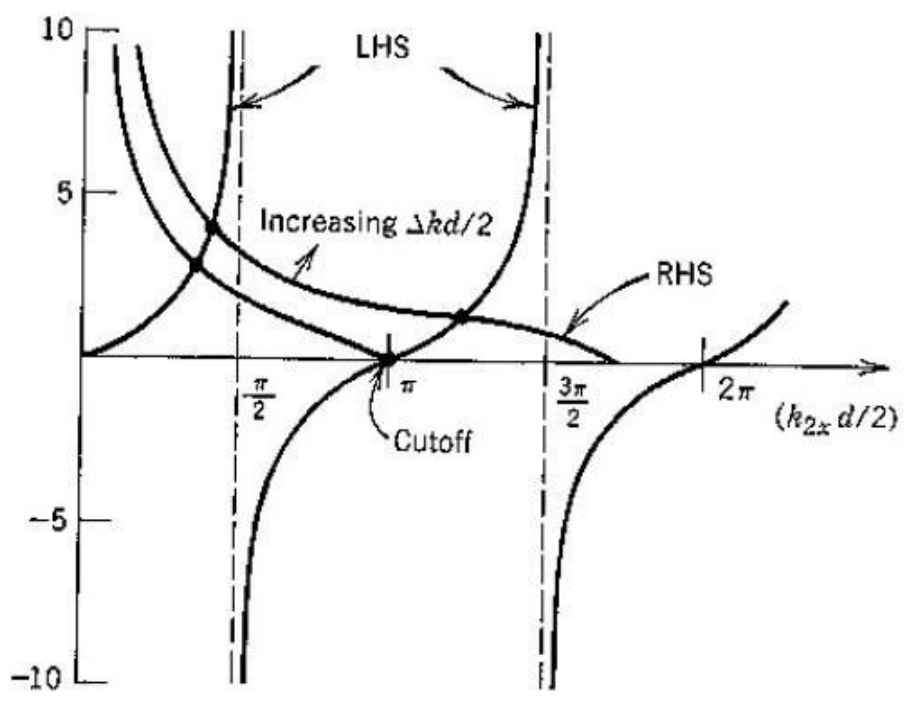

The guidance condition expressed graphically for even modes.

Fig2. 4 Graphical solution for guiding condition

Assuming the amplitude of the guiding wave in the waveguide is unity and equating both the equations at $\mathrm{x}=\mathrm{d} / 2$ we get $C_{1}=\cos \left(k_{2 x} d / 2\right)$. Hence the final field equations for even modes become

$$
\begin{aligned}
E_{y}(x) & =\cos \left(k_{2 x} d / 2\right) \exp \left(-\alpha_{1}(x-d / 2)\right) & \mathrm{x}>\mathrm{d} / 2 \\
& =\cos \left(k_{2 x} x\right) & -\mathrm{d} / 2<\mathrm{x}<\mathrm{d} / 2 \\
& =\cos \left(k_{2 x} d / 2\right) \exp \left(\alpha_{1}(x-d / 2)\right) & \mathrm{x}<-\mathrm{d} / 2
\end{aligned}
$$


Adopting similar procedure and using 'sin' instead of 'cos' in Eq 2.10 we get the solutions for odd modes. Odd field solutions are

$$
\begin{array}{rlr}
E y(x) & =\sin \left(k_{2 x} d / 2\right) \exp \left(-\alpha_{1}(x-d / 2)\right) & \mathrm{x}>\mathrm{d} / 2 \\
& =\sin \left(k_{2 x} x\right) & -\mathrm{d} / 2<\mathrm{x}<\mathrm{d} / 2 \\
& =-\sin \left(k_{2 x} d / 2\right) \exp \left(\alpha_{1}(x-d / 2)\right) & \mathrm{x}<-\mathrm{d} / 2
\end{array}
$$

and the guiding condition for the odd modes is

$$
\cot \left(k_{2 x} d / 2\right)=-\frac{\alpha_{1}}{k_{2 x}}
$$

\subsubsection{Cutoff Conditions:}

There would be more than one solution for Eq 2.15 and the number of solutions gives the number of even modes. From Eq 2.15 we can determine the upper and lower guidance frequency limits known as cutoff conditions. For a pth mode to exist [20]

$$
p \pi<\left(k_{2 x}\right)_{p} d / 2<\left(p+\frac{1}{2}\right) \pi
$$

Hence if $\left(k_{2 x}\right)_{p} d / 2<p \pi$, the $\mathrm{p}^{\text {th }}$ mode would be lost. This is known as the lower cutoff frequency limit and if $\left(k_{2 x}\right)_{p} d / 2 \rightarrow(p+1 / 2) \pi$, the fields are contained entirely in the dielectric core and hence this limit is known as the upper cutoff frequency. In terms of ray optics, lower cutoff represents the mode angle nearer to critical angle, below which the total internal reflection doesn't take place and upper cutoff implies an angle of $90^{\circ}$ which indicates propagation is parallel to the surface of the waveguide.

\subsection{Analysis of Asymmetric Waveguides:}

Assuming conditions similar to symmetrical waveguides we end up with electric fields of the form

$$
\begin{array}{rlrl}
E_{y} & =C_{1} e^{-\alpha_{1 x} x} & \mathrm{x}>\mathrm{d} / 2 \\
& =C_{2} \cos \left(k_{2 x} x+\psi\right) & -\mathrm{d} / 2<\mathrm{x}<\mathrm{d} / 2 \\
& =C_{3} e^{\alpha_{3 x} x} & \mathrm{x}<\mathrm{d} / 2
\end{array}
$$

Where $\alpha_{1 x}=\sqrt{k_{z}^{2}-\omega^{2} \mu_{1} \varepsilon_{1}}, \alpha_{3 x}=\sqrt{k_{z}^{2}-\omega^{2} \mu_{3} \varepsilon_{3}}$ and $k_{2 x}=\sqrt{\omega^{2} \mu_{2} \varepsilon_{2}-k_{z}^{2}}$ 
Since the waveguide is not symmetric the solutions would not be either even or odd and hence we have a phase constant in the cosine term in the waveguide region [20]. Also since the waveguide is not symmetric we have three amplitudes for three regions. From Eq 2.11, we get

$$
\begin{aligned}
H_{z} & =\frac{-j \alpha_{1 x}}{\omega \mu_{1}} C_{1} e^{-\alpha_{1 x} x} \\
& =\frac{-j k_{2 x}}{\omega \mu_{2}} C_{2} \sin \left(k_{2 x} x+\psi\right) \\
& =\frac{j \alpha_{3 x}}{\omega \mu_{3}} C_{3} e^{\alpha_{3 x} x}
\end{aligned}
$$

Applying boundary conditions for electric and magnetic fields at $\mathrm{x}=\mathrm{d} / 2$ similar to symmetric waveguides we get [20]

$$
\tan \left(k_{2 x} d / 2+\psi\right)=\frac{\mu_{2} \alpha_{1 x}}{\mu_{1} k_{2 x}}
$$

Similarly applying boundary conditions at $x=-d / 2$, we get

$$
\tan \left(k_{2 x} d / 2-\psi\right)=\frac{\mu_{2} \alpha_{3 x}}{\mu_{3} k_{2 x}}
$$

Eliminating $\psi$ we get

$$
2 k_{2 x} d-2 \tan ^{-1}\left(\frac{\mu_{2} \alpha_{1 x}}{\mu_{1} k_{2 x}}\right)-2 \tan ^{-1}\left(\frac{\mu_{2} \alpha_{3 x}}{\mu_{3} k_{2 x}}\right)=2 p \pi \quad p=0,1 \ldots
$$

Eq 2.22 is the generalized guiding condition and this could be solved either graphically or numerically to obtain the modes. Here $p$ denotes the mode number. Unlike symmetric waveguides, the mode profile in asymmetric waveguides is not symmetric along the waveguide axis. Chapter 3 discusses more about the mode profile and the effect of cladding indices on it.

\subsection{Waveguide Attenuation:}

Our device operation depends on the evanescent tail and the power observed at the ends of the waveguide. Hence it is necessary that appreciable power reaches the ends of the waveguide. The reduction in the waveguide power present at the ends of the 
waveguides relative to that launched in the guide is due to attenuation. Hence it is important to understand attenuation and its causes, so that their effects can be minimized and attenuation reduced.. Attenuation is the measure of loss between input and output ends. Coupling, scattering, absorption and radiation losses are important sources of attenuation in waveguides. Coupling losses are present at the ends of the waveguide while the scattering and absorption losses occur along the guides length. Radiation losses depend on the geometry of the waveguide surface.

\subsubsection{Absorption Losses:}

Materials absorb light energy depending upon the operating wavelength and its material characteristics. If the absorption is in the visible spectrum then it is opaque. As light passes through the waveguide, it may be absorbed by the waveguide material. This is known as intrinsic absorption. Impurities within the waveguide would result in extrinsic absorption. Absorption loss results from the electronic and vibrational resonances associated with molecules of the guiding and nearby cladding material [23]. Care has to be taken that the materials chosen do not have absorption peaks at the operating wavelengths. For example the hydroxyl ion has an absorption peak at 1300$1400 \mathrm{~nm}$ range and would result in a maximum loss at that wavelength. Neglecting the loss from impurities, absorption loss is uniform and constant. A fixed amount of material would absorb the same fraction of light passing through it. Hence the loss can be termed cumulative. If $\alpha$ is the absorption loss coefficient (in $\mathrm{dB} / \mathrm{cm}$ ) then the fraction of light remaining after traveling a distance ' $\mathrm{d}$ ' $\mathrm{cm}$ can be found to be $(1-\alpha)^{\mathrm{d}}$. Absorption losses can be reduced to a large extent by suitable choice of material and operating wavelength. In optical fibers these losses are prominent as the operating wavelength is around $1300 \mathrm{~nm}$ and glass has an absorption peak in that region. In spite of this, fiber optic communications operate at those wavelengths due to other factors like material dispersion and phase shift. 


\subsubsection{Scattering Losses:}

Scattering losses typically form the major part of all the losses and have to be minimized for better operation of any waveguide device. There are many types of scattering losses based on their origin. Only the most prominent are dealt with here. There are two types of scattering losses based on the output frequency. If the output frequency remains the same as the input frequency and if only the power is reduced then it is linear scattering. The loss of power would be due to the scattering of power from a guided mode to another guided mode or a radiative mode thus losing the guided power. This type of scattering would produce only attenuation and the output frequency remains the same as that of the input. Rayleigh scattering and Mie scattering are of this type. Non linear scattering results in change of some of the input power to another frequency. Brillouin scattering and Raman scattering are of this type.

\subsubsection{Rayleigh and Mie Scattering:}

Atoms are deposited randomly during deposition and the substrate heat provides the energy for the lateral motion at the surface. The random deposition of the atoms might result in local density variation and thus change the refractive index. This can be visualized as scattered refractive indices in a homogeneous medium whose size would be comparable to the wavelength of light. When light propagates through such a medium it gets scattered and hence power is lost into radiation modes or other modes. This type of scattering is known as Rayleigh scattering. There are several methods to analyze these losses. Rayleigh scattering depends on the intensity and wavelength of the light passing in the waveguide. Scattering might also result from imperfections on the waveguide surface and this type of scattering is termed to be Mie Scattering. The Rayleigh scattering is proportional to the wavelength of light $\left(\lambda^{-4}\right)$ and to the light intensity propagating in the waveguide. In a fiber if $P(r)$ is the intensity of the light and ' $r$ ' is the radial distance then loss is given by [24]

$$
S_{1}=\lambda^{-4} * \int_{0}^{\infty} A(r) P(r) r d r / \int_{0}^{\infty} P(r) d r
$$

where $A(r)$ is the Rayleigh scattering coefficient at radial distance ' $r$ '. From the above equation we can see that the loss at a point is dependent on the optical power at that point 
and the Rayleigh scattering coefficient The Rayleigh scattering in a waveguide can be approximated by the expression [25]

$$
S_{L}=1.7 *(0.85 / \lambda)^{4}
$$

The average Rayleigh scattering loss of a single mode waveguide is given by [26]

$$
\bar{\partial}_{s}=\iint_{A} \partial_{s}(x, y) E^{2}(x, y) d x d y
$$

where $\partial_{s}(x, y)$ is the Rayleigh scattering loss at the point $(\mathrm{x}, \mathrm{y})$ in the waveguide cross section and is defined as

$$
\begin{aligned}
\partial_{s}(x, y) & =C_{1}\left[n^{2}(x, y)-1\right] / \lambda^{4} & & \text { Core } \\
& =C_{2}\left[n_{2}^{2}-1\right] / \lambda^{4} & & \text { Cladding }
\end{aligned}
$$

where $\mathrm{C}_{1}$ and $\mathrm{C}_{2}$ are the Rayleigh scattering coefficients of the core and the cladding respectively. From the equations it is evident that Rayleigh scattering loss can be reduced if operated at higher wavelengths.

\subsubsection{Nonlinear Scattering:}

Nonlinear scattering results in change of the output frequency and the power would be attenuated at the input frequency but amplified at the other frequency. There could be several nonlinear effects that would result in scattering of the power either to the same propagating mode or to the other propagating mode at different frequency. The types of nonlinear scattering losses are Raman Scattering and Brillouin Scattering and both of these losses occur at high optical power levels.

\subsubsection{Radiation Losses or Bending Losses:}

Radiation losses occur at bends or curves of the waveguide paths. These losses usually occur as the evanescent tails' velocity exceeds the velocity of light in the cladding and hence the light is radiated from the waveguide. As Fig 2.5 illustrates, during bends evanescent tail in the cladding region has to travel faster than the light in the core to stay with the wave which is not possible and hence that part of light is lost in the radiation modes. The radiation attenuation coefficient is given by [22]

$$
\alpha_{R}=c_{1} \exp \left(-c_{2} R\right)
$$


Where $\mathrm{R}$ is the radius of curvature of the waveguide bend and $c_{1}$ and $c_{2}$ are constants that depend upon the geometry. Bending losses can be reduced by employing large refractive index differences and shorter wavelength operation.

Radiation losses occur in straight waveguides when scattering events couple optical power in guided modes into radiation modes which then carry the power from the waveguide.

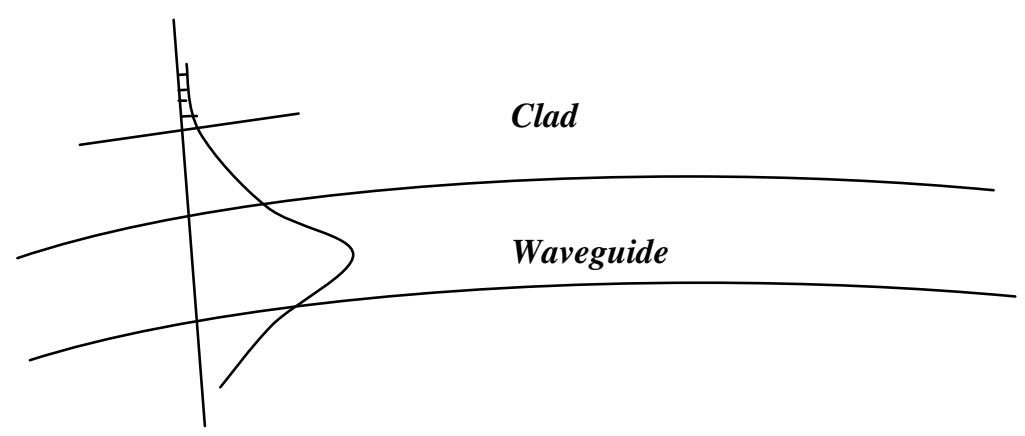

Fig2. 5 Radiation losses at the bends

\subsubsection{Coupling Losses:}

Losses that occur during coupling of light to and from the waveguide are termed coupling losses. These can be reduced only by using efficient couplers and the losses would vary with the optical schemes employed to input light into the waveguides. These losses do not depend on any of the waveguide parameters but depend on the physcial operation of the device. As an example losses in prism coupling can be reduced by coupling the light at the coupling spot and thus enabling maximum amount of power transfer into the waveguides.

In thin dielectric waveguides the major source of attenuation is through scattering. Hence all the losses are usually neglected while measuring losses in the waveguide. Assuming the attenuation present only due to scattering the intensity at a point in the propagation of the waveguide can be approximated by the following equation for all the practical purposes. 


$$
I(z)=I_{0} \exp (-\alpha z)
$$

Where $I_{0}$ is the initial intensity at $\mathrm{z}=0$, and the loss in $\mathrm{dB}$ is related to $\alpha$ by the following expression [22].

$$
\operatorname{Loss}(\mathrm{dB} / \mathrm{cm})=4.3 \alpha\left[\mathrm{cm}^{-1}\right]
$$

\subsection{Prism Coupler [28]:}

Prism coupler is a device used to couple light into the waveguides and determine the refractive index and thickness of the guiding film, provided that the film supports two or more modes. Thickness and index are obtained by measuring the coupling angles at the prism and fitting them to the dispersion curve. Frustrated total internal reflection takes place between the prism and film interface which allows light to travel into the thin film.

\subsubsection{Frustrated Total Internal Reflection:}

When light is incident from a higher index material to a lower index material, there is total internal reflection if the incident angle exceeds critical angle. Though there is no power in the second medium there is an evanescent wave which decays rapidly. If there is a third medium which is optically denser than the second medium, and if the thickness of second medium is not large enough to decay the evanescent wave (total internal reflection gets frustrated) then the power from first medium is transferred to the third medium. This phenomenon is known as frustrated total internal reflection. It is similar to that of quantum mechanical tunneling across two narrow potential barriers.

Fig 2.6 shows a laser beam (light source) that hits the high indexed prism during which some of the light passes into the film through the air gap. Prism Coupler operation is based on this principle. 


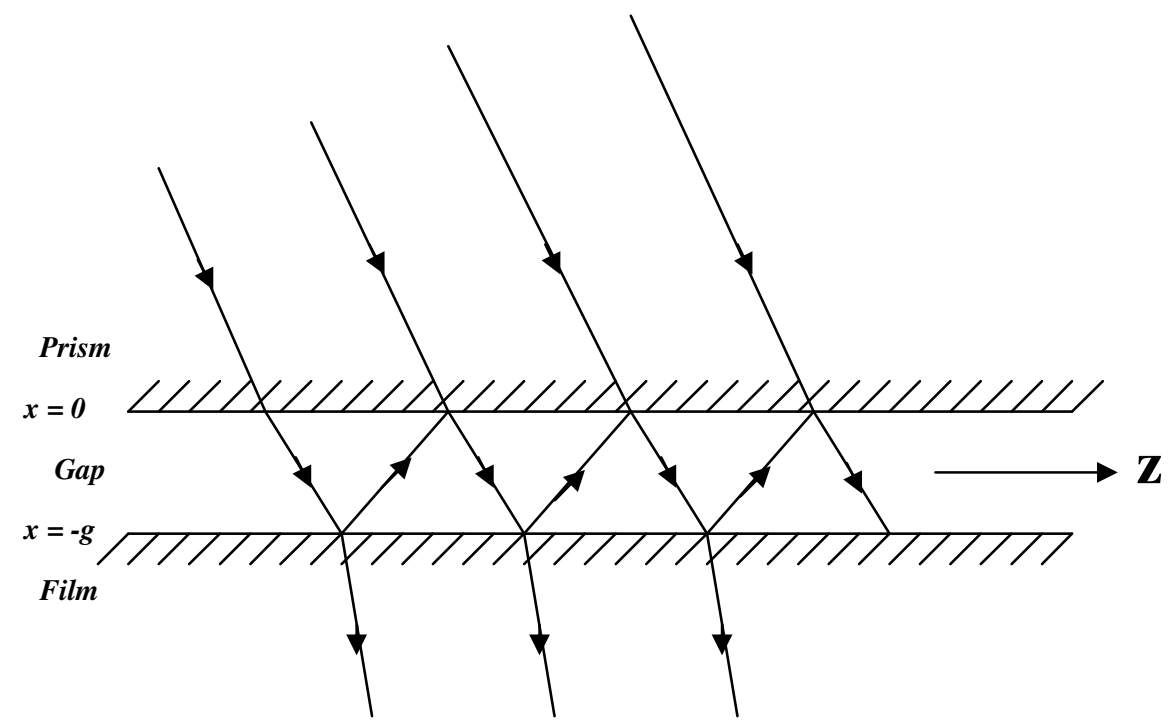

Fig2. 6 Contribution of several incident rays to the transmitted wave

\subsubsection{Prism Coupling and Effective index [28]:}

Every mode in a waveguide, depending upon the medium it is present in, has a distinct angle at which the light propagates. If light is incident onto the prism at an angle greater than critical angle, total internal reflection takes place and the light gets reflected as shown in Fig 2.4. There is also an evanescent wave that goes into the second medium, here air, and if the evanescent waves' velocity matches with the phase velocity of a mode of the waveguide then the light would propagate through the waveguide along the matched modal path. This phase velocity is reached only at certain distinct positions which are termed to be mode angles. At these angles photons travel through the air gap into the film and enter into an optical propagation mode which corresponds to sudden dip in the intensity levels observed in the reflected wave. Fig 2.8 shows a general intensity image obtained when SPARROW based structure is deposited on Borofloat wafer and is checked for modes in the Prism Coupler. It is understood that the film is placed very near to the prism and the distance between them is less than the wavelength of light. Since TM and TE waves have different modal angles the evanescent wave from the prism should also be a polarized wave to observe this phenomenon. 


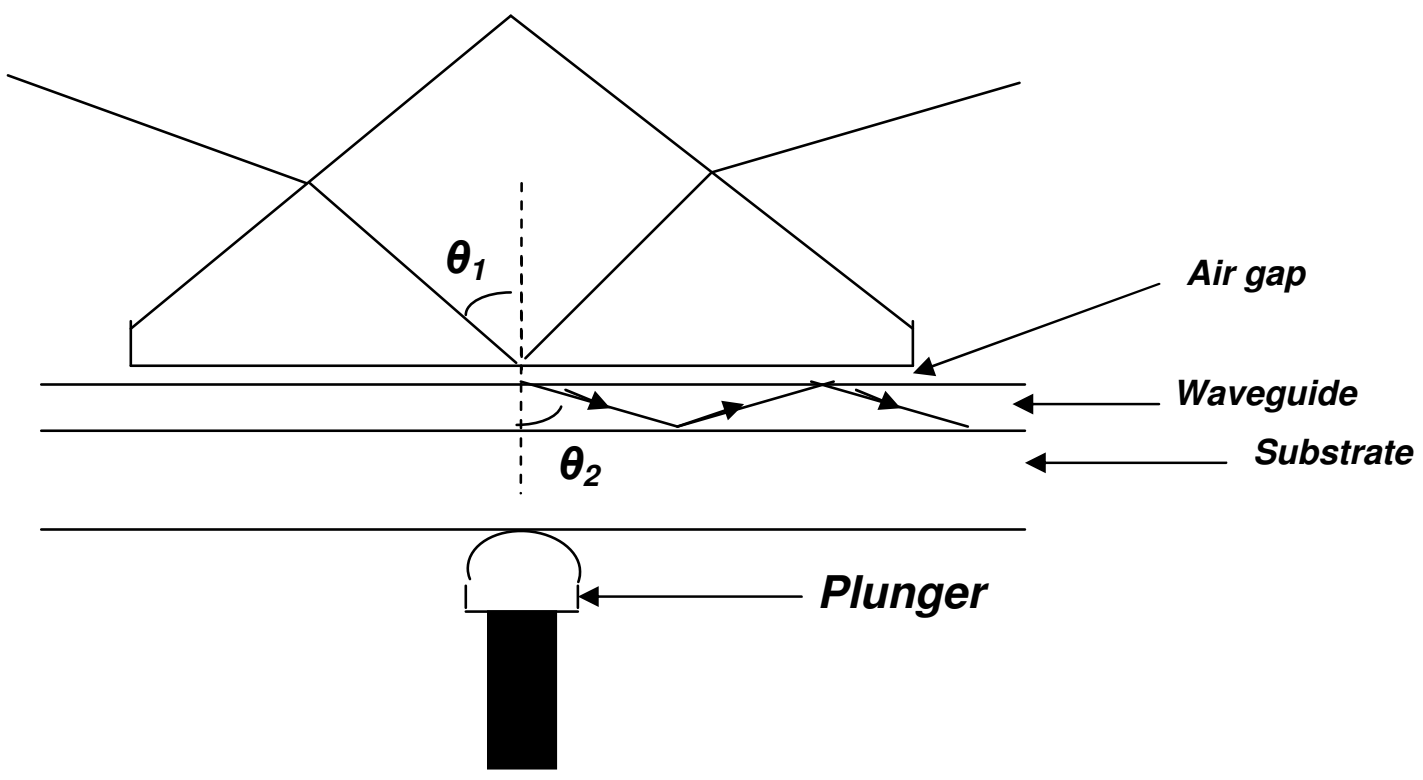

Fig2. 7 Prism coupler setup [27]

The modal equation is given by [28]

$$
(2 \pi / \lambda) * \cos \left(\theta_{2}\right) * d+\Phi_{1}+\Phi_{2}=m \pi \quad(m=0,1,2 \ldots)
$$

where $\lambda$ is the operating wavelength, $d$ is the thickness of the waveguide, $\Phi_{1}$ and $\Phi_{2}$ are the phase shifts at the air film interface and film substrate interface respectively. This is similar to Eq 2.22 but written in different form. When two modal angles are known then the parameters like thickness and index can be measured from the above equation. If the film supports only a single mode then either the thickness or the index of the film should be provided to calculate the other parameter.

Rays traveling in each mode angle have a corresponding modal index which is also known as effective index. This is given by [28]

$$
N(\theta)=n_{p} \sin \left(\theta_{1}\right)
$$

where $n_{p}$ is the refractive index of the prism and $\theta_{1}$ is the angle it makes with the base of the prism. More generally the effective index is $\operatorname{simply} n \sin (\theta)$ where $\theta$ is the angle of incidence the light makes at the boundary of the material. Since from Snell's law

$$
n_{1} \sin \left(\theta_{1}\right)=n_{2} \sin \left(\theta_{2}\right)
$$

where $\mathrm{n}_{1}, \theta_{1}$ are refractive index and angle of incidence in medium 1 respectively and the same with the other medium. When the rays enter the waveguide it obeys the Snell's law. 
Hence the quantity $n_{p} \sin (\theta)$ remains the same and this quantity is maintained for all the transitions. Hence we get

$$
n_{p} \sin \left(\theta_{1}\right)=n_{w g} \sin \left(\theta_{2}\right)
$$

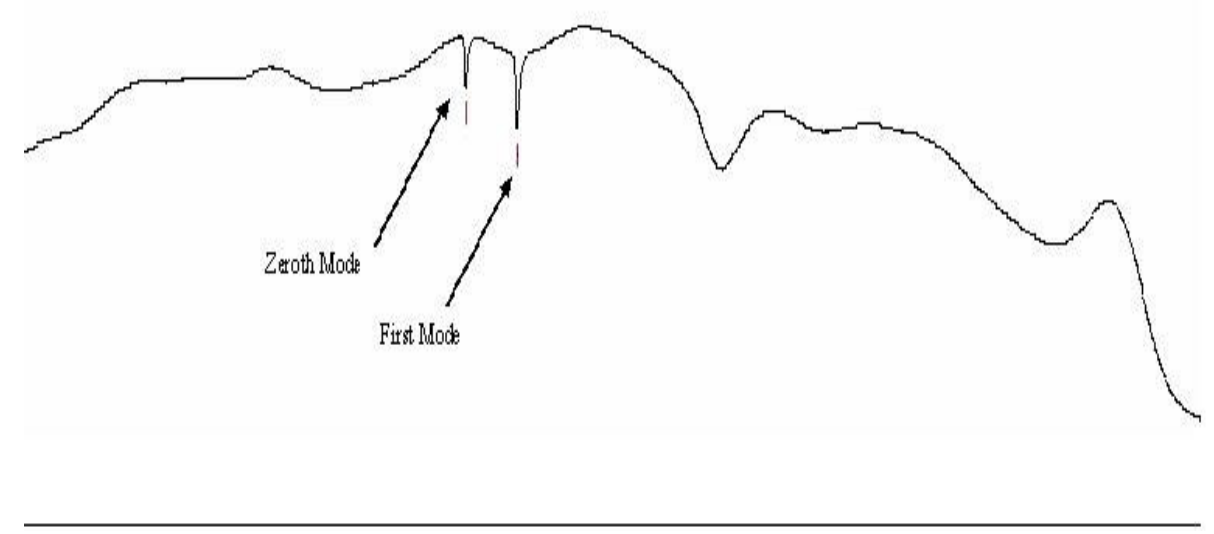

Fig2. 8 Intensity plot showing two modes of the designed structure

From the above expression we can conclude that the value $N(\theta)$ remains the same. During modal angles the light enters into the waveguide and the corresponding $N(\theta)$ is said to be the effective refractive index $\left(n_{\text {eff }}\right)$ of the guiding wave.

\subsubsection{Prism Coupler Input Firing:}

Prism coupler and butt coupling are the general techniques used to couple light into the waveguides. Prism coupling is an optical technique developed to couple light into the waveguides based on Frustrated Total Internal Reflection with minimum stress on the film. Mode selectivity is not possible through butt coupling while it is possible through prism coupling. The device architecture adopted in this work has coupled waveguides and a single waveguide has to be excited at a time for the device operation. Prism coupler can thus be helpful in exciting a single mode. Hence Prism coupler has been chosen for exciting the waveguides.

Light couples at an angle ' $\theta$ ' at which the propagation velocity in the prism and air gap is equal to the phase velocity corresponding to a mode in the waveguide. This angle can be used to obtain the effective index of the medium and further can be used to 
calculate the beta or the propagation constants of the guiding wave. Hence this forms the good reference to validate the results obtained from the simulation models. Also optical waveguide loss measurements can be done using prism coupler along with a CCD camera. This technique was employed to compute the losses in the waveguide. Details about this technique are given in Appendix B.

\subsection{Coupled Mode Theory:}

When light propagates in a waveguide there is power coupled from one mode to the other mode. Also there would power transfer if another waveguide is bought adjacent to an excited waveguide. Coupled mode theory can be used to explain this behavior. Our device has two resonant waveguides and coupled mode theory explains the theory behind this resonance.

Coupled mode theory has been extensively applied as a tool to analyze the electromagnetic wave propagation in integrated optics. The propagation parameters of an electromagnetic wave in the waveguide changes at perturbations and this can be explained using this theory. Initially this has been used in microwave kinetics and later has been applied to guided optics by Snyder, Marcuse, Yariv and Kogelnik in 1970's. The analysis of coupled waveguide systems is based on the modes, propagation constants and amplitudes of the individual waveguides [31]. If mode profiles are independent of each other (orthogonal) then orthogonal coupled mode theory applies, else it is nonorthogonal. If the device consists of weakly coupled waveguides, similar propagation constants then orthogonal coupled mode theory can be applied. In both the cases the solution is obtained from the linear superposition of the waveguide modes [31]. Consider two guided modes which have the amplitudes $a_{1}$ and $a_{2}$ respectively with the implicit time dependence $\exp (j \omega t)$. If they are infinitely apart from each other then they would obey the equations [30]

$$
\frac{d a_{1}}{d z}=-j \beta_{1} a_{1} \quad \frac{d a_{2}}{d z}=-j \beta_{2} a_{2}
$$

Where $\beta_{1}$ and $\beta_{2}$ are the propagation constants and will remain the same. If they are brought close, the evanescent fields would interact with each other and hence mode coupling occurs. If the coupling is weak then the equations would become 


$$
\begin{aligned}
& \frac{d a_{1}}{d z}=-j\left(\beta_{1}+K_{11}\right) a_{1}-j K_{12} a_{2} \\
& \frac{d a_{2}}{d z}=-j\left(\beta_{2}+K_{22}\right) a_{2}-j K_{21} a_{1}
\end{aligned}
$$

Where $\left(\mathrm{K}_{12}, \mathrm{~K}_{21}\right)$ and $\left(\mathrm{K}_{11}, \mathrm{~K}_{22}\right)$ are the mutual and self coupling coefficients respectively. If the waveguides are lossless then the total power has to be maintained constant. Hence,

$$
P(z)=\left|a_{1}\right|^{2}+\left|a_{2}\right|^{2}
$$

From the power conservation we get

$$
\frac{d}{d z} P(z)=0
$$

Solving 2.33 we get

$$
K_{12}=K_{21}^{*}=\kappa
$$

For the uniform couplers both the propagation constants and the coupling coefficients are independent of $\mathrm{z}$ and hence equations 2.4 can be solved analytically. For simplification we assume

$$
a_{i}(z)=\stackrel{\Lambda}{a}_{i} \exp \left(-j \frac{\beta_{1}+K_{11}+\beta_{2}+K_{22}}{2} z\right)
$$

Hence Eq (2.30) can be rewritten as

$$
\begin{aligned}
& \frac{d \hat{a_{1}}}{d z}=-j \delta \hat{a_{1}}-j \kappa \hat{a}_{2} \\
& \frac{d \hat{a_{2}}}{d z}=+j \delta \hat{a}_{2}-j k \hat{a_{1}}
\end{aligned}
$$

Where $\delta=\frac{\beta_{1}+K_{11}-\beta_{2}-K_{22}}{2}$

Rewriting the above in the matrix form we get

$$
\frac{d}{d z} A=-j \bar{H} A
$$

Where $A=\left[\begin{array}{l}\hat{a}_{1} \\ \hat{a}_{2}\end{array}\right]$ 
And $\mathrm{H}=\left[\begin{array}{cc}+\delta & \kappa \\ \kappa & -\delta\end{array}\right]$ After diagnolization by unitary matrix such that $\mathrm{O}^{\mathrm{t}} \mathrm{HO}=\mathrm{B}$ where $\mathrm{B}$ is the diagonal matrix, $\mathrm{Eq} 2.36$ is obtained. Here $\beta_{s}$ and $\beta_{a}$ are the symmetric and antisymmetric modes of the combined structure.

$$
\left[\begin{array}{cc}
\beta_{s} & 0 \\
0 & \beta_{a}
\end{array}\right]
$$

Thus the beta values of the composite modes can be written as

$\beta_{s}=\beta_{0}+S$ and $\beta_{a}=\beta_{0}-S$ where $S=\sqrt{\delta^{2}+\kappa^{2}}$ and $\beta_{0}=\frac{\beta_{1}+K_{11}+\beta_{2}+K_{22}}{2}$.

Assuming only one waveguide is excited then $a_{1}=1$ and $a_{2}=0$, the power in waveguides can be represented by

$$
P_{1}(z)=\cos ^{2}(S z)+\cos ^{2}(\eta) \sin ^{2}(S z) \quad P_{2}(z)=\sin ^{2}(\eta) \sin ^{2}(S z)
$$

Where

$$
\eta=\tan ^{-1}\left(\frac{\kappa}{\delta}\right)
$$

At $\mathrm{z}=\mathrm{L}_{\mathrm{c}}$ maximum power transfers from guide 1 to guide 2 and is known as coupling length. It is given by

$$
L_{c}=\frac{\pi}{2 S}
$$

Coupling length is the distance at which maximum power transfer occurs from one waveguide to the other waveguide. Higher coupling lengths means that the power transfer rate is slow. A lower coupling length model would be prone to noise depending upon the coupling length a suitable interaction length for sensing would be selected. A higher interaction lengths and lower coupling lengths would result in lot of power transfers from one waveguide to other and may result in higher losses.

\subsection{Beam Propagation Method:}

The Beam Propagation Method (BPM) is one of the most powerful techniques employed to investigate linear and non-linear light wave propagation in waveguides. Devices such as curvilinear directional couplers, branching and combining waveguides, 
etc. can be simulated by BPM. Like Finite Difference Time Domain method, finite difference BPM solves Maxwell's equations by using finite differences in place of partial derivatives. It differs from FDTD in two aspects. Firstly BPM is done entirely in the frequency domain. Secondly it uses a slowly varying envelope approximation in the direction of light propagation. There is not much change in terms of refractive index or thickness in the propagation direction and this suits our device design.

\subsubsection{Slow Varying Envelope Approximation:}

If $X$ is electrical or magnetic component of electromagnetic field, it changes most rapidly along the optical axis. The slowly varying approximation involves replacing the quickly varying component, $X$, with a slowly varying one, $x$

$$
X(x, y, z)=x(x, y, z) \exp \left(-j k n_{o} z\right)
$$

Where ' $n_{o}$ ' is known as reference index. If light is traveling almost parallel to the axis (paraxial approximation), and is monochromatic, then it is possible to select a reference index $n_{o}$ which makes $X$ a slowly varying function in all three directions [29]. If $x$ is slowly varying it is possible to choose fewer mesh points to improve the speed of calculation without compromise in accuracy. Hence accurate and efficient results are possible for step sizes many times greater than optical wavelength along the propagation direction within less calculation time.

Eq 2.39 can be used to approximate the size of the mesh. As $x$ varies much slower than $X$, significant savings in calculation time can be obtained. If the problem cannot be reduced then $x$ will need to vary more quickly, and the mesh size should be reduced in all directions more importantly in propagating direction. In general, simulations for different mesh sizes have to be performed and compared for consistency. Finer mesh would give accurate results but would take longer simulation times. Models which have large index contrast in $x-y$ plane, thin layers are to be simulated with higher mesh values.Our device design has been simulated for different mesh sizes ranging from 106 points/ $\mu \mathrm{m}$ to 1720 points/ $\mu \mathrm{m}$ and consistent results have been obtained. Again the simulation time for higher mesh points was also not high and adopting finer mesh would be better choice for calculations. 
There are few assumptions that light propagates in a single direction and the reflection is not allowed in slowly varying envelope method. Hence suitable boundary conditions are to be implemented to keep the reflection effect to a minimum.

OPTIBPM 7 simulator assumes the clad index as that of wafer on either side of the waveguide during the creation of an input wave which is not true. '2d Mode solver' has to be adopted to get exact input wave. The input cannot be given at our choice as the simulator is designed to give input at the center of the wafer. Thickness of the substrate and cladding has to be adjusted to give input to the waveguide of our choice and accordingly similar design has to be adopted in mode solver to create the input wave. More discussion about the simulator is present in Appendix A. 


\section{CHAPTER 3: Modeling and Fabrication}

Chapter 2 discusses the theoretical analysis of optical modes in a waveguide. Here the change of mode profiles with waveguide thickness and the importance of the length of evanescent tail in bio-sensing applications are discussed. We will also discuss the modeling of biosensor, PMMA patterning for measurement window, silicon master fabrication for PDMS channels and bonding of PDMS channel to alumina surface.

\subsection{Mode Profile Variation}

The optical mode profile in dielectric slab waveguides is dependent on the thickness and index of the waveguide and also on the index contrast between the waveguide and the cladding/substrate. Fig 3.1 shows the modal profile of the structure shown. Fig 3.1a is a symmetric waveguide with index of 1.7 and $200 \mathrm{~nm}$ in thickness with clad index of 1 on both sides. The mode profile is also symmetric with same penetration depth of $53 \mathrm{~nm}$ on both sides. Fig 3.1b is an asymmetric waveguide with the same parameters of that of symmetric waveguide except that one of the cladding indices is 1.4. The profile is asymmetric and penetration depth (1/e distance from waveguide surface) is also not the same on both sides. The penetration depths are $146 \mathrm{~nm}$ on the higher index side (1.4) and 40nm on the lower index side (1.0). Observing Fig 3.1b the optical field intensity drops more steeply when the refractive index contrast is large. A higher index contrast results in steep decrease in intensity and lower penetration depth.
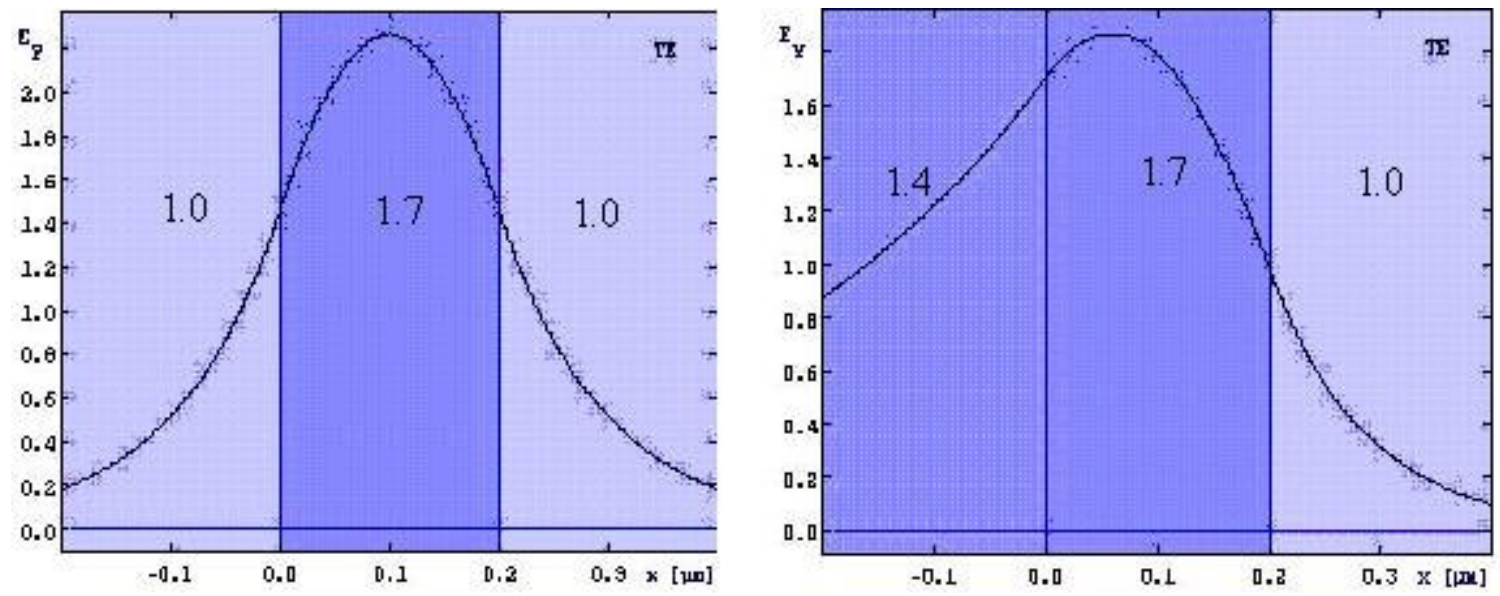

Fig 3. la\&b Intensity Variation in symmetric and asymmetric waveguides. 
The biosensor of the device concept discussed here is based on evanescent wave detection. Fig 3.2 shows a general model of the same. The probe area of the sensor usually is composed of the biolayer and the analyte with agents to be detected. The area within the penetration depth, $d_{p}$ can be used for bio-sensing applications. Generally the length of the biolayer varies anywhere from 40nm-120nm depending upon the organic chain selected and the bio-agent to be detected. The biolayer serves to perform molecular recognition when the probe molecule within it binds with the targeted analyte in solution. It also serve the important function of inhibiting nonspecific binding to the sensor surface. For better operation of the device, the ratio of the interaction volume of the bioagent targets, once bound to the bio-layer relative to the evanescent wave modal volume in the region should be as high as possible. Hence the bio-layer chosen should be smaller in length and also the model designed should give larger penetration depths. The device architecture used here has an evanescent tail of 100nm while the bio-layer we use has a maximum thickness of $40 \mathrm{~nm}$ prior to analyte binding. Hence we have approximately $60 \mathrm{~nm}$ of usable area present in the probe area.

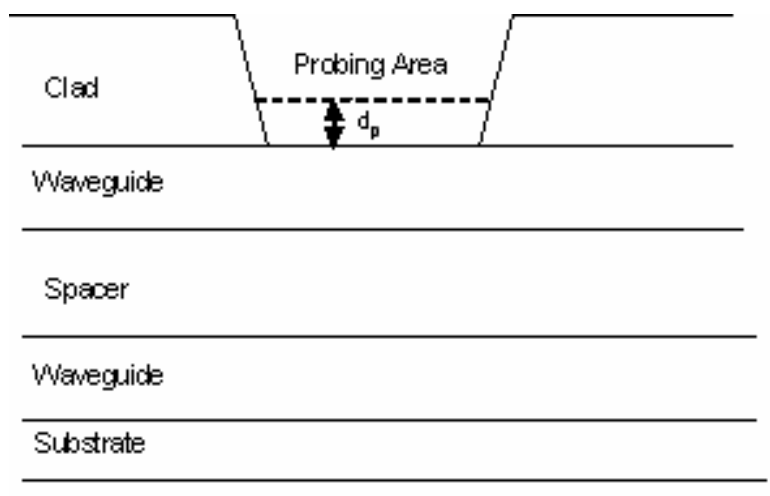

Fig 3. 2 Biosensor design and penetration depth

\subsection{Modeling of SPARROW Biosensor:}

Accurate analysis of optical waveguides is required to effectively develop integrated photonic waveguide devices. Optical field variation in coupled waveguides and power transfer are difficult to solve in closed form for waveguides which are highly asymmetric as it involves second order differential equations. A good modeling technique is needed to simulate the fabricated coupled waveguides and calculate the optical 
parameters. 'OptiBPM' is a simulation tool based on Beam Propagation Method (BPM) and performs step by step simulation of light propagation in a waveguide. The optical field at any point of propagation can be calculated using this software. It also performs simulations faster when compared to FDTD (Finite Difference Time Domain). Due to the paraxial approximations assumed in BPM, the simulator can be used for structures involving waveguides and other structures where backside reflection is not considered. Simulator might not work well curved waveguides and devices which are based on backside reflection. Device structure in discussion ideally does not have backward reflections and hence BPM simulations can be used. So the simulations for the tuned structures have been done using OPTIBPM 7.0. A Finite difference Beam Propagation Method is adopted to solve the Helmholtz equations at every mesh point.

Fig 3.2 shows the SPARROW (stacked planar affinity regulated resonant optical waveguide) sensor of this study. During device operation, the light coupled into a waveguide continues to propagate in the waveguide in which it was launched until it enters the probe area where the analyte solution is present above the waveguide stack. At this point the two stacked waveguides are in resonance and exchange power when no analyte is bound to the biolayer. A complete power transfer occurs at the probe area due to the refractive index of analyte solution. If adequate bio-agent is attached to the biolayer surface, there would not be complete power transfer and based on the power observed at the ends of the waveguide the presence of the attached bioagent can be determined. Hence our design should be such that light interacts with all the interfaces as shown in Fig 3.4a and the goal is that optical power transfer at PDMS/air interface is ideally zero while a complete power transfer or resonance is observed in the channel/probe area. The simulations have been performed assuming the channel's refractive index to be varying from 1.3318 to 1.43 . Usually the biolayer grown in the channel has an index of 1.4-1.5 and the effective index when present in analyte (here water) can vary from 1.3318-1.43 depending upon the percentage coverage of the Biolayer. Practically for testing purposes without the biolayer, these indices can be attained using different concentrations of water and glycerol. 
There are many ways to create the input wave for the simulations. Since the prism coupler is used to couple light into waveguides, a modal field is chosen as input wave for the simulations. More details about the input types and the assumptions in the simulation software can be found in [29]. The modal input used in the simulations is generally the intensity distribution wave of the whole structure and can be obtained using any mode solving tools. We used ' $2 \mathrm{~d}$ Modesolver' to obtain the modes of the simulated structure. ' $2 \mathrm{~d}$ Modesolver' solves for the modes in a given structure and those modal intensities can be used as input wave for the simulations. Fig (3.3a) is a sample structure and Fig $3.3 \mathrm{~b}$ is the modal field distribution obtained using the mode solver tool. Most of the power is concentrated in the top waveguide. This wave is given as input to Fig 3.3a. Fig 3.4 shows the design that is used for the simulations and the optical field intensity distribution for the structure is shown in Fig 3.5.

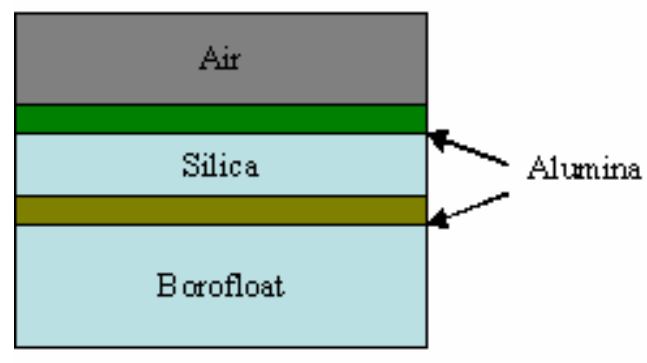

Fig 3. 3a Model for simulation

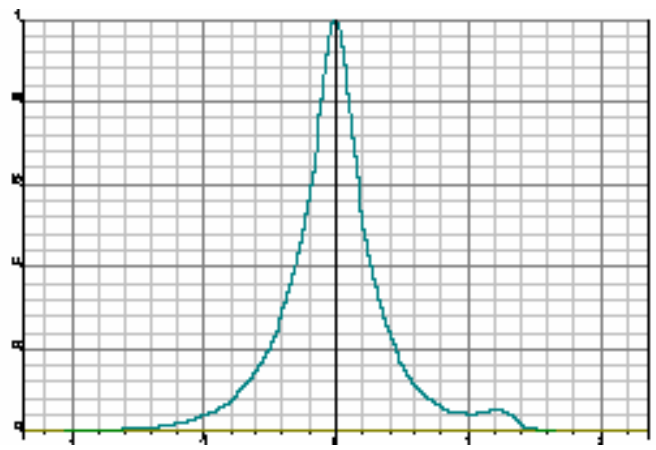

Fig 3.3b Optical Field Distribution

As discussed earlier the field should be confined to a single waveguide if the interfacing layer for the top waveguide is air or PDMS but should allow power transfer when the medium is analyte (here water). Fig 3.5 shows the simulated optical field intensity variation for the design shown in Fig 3.4. It is observed that the optical power enters the bottom waveguide, continues to propagate in the waveguide at both PDMS and air interfaces and complete power transfer occur at the water interface. A little scattering occurs at the interfaces and this is due to the sudden change in the medium. 


\begin{tabular}{|c|c|c|c|c|}
\hline Air & PDMS & Water & PDMS & Air \\
\hline \multicolumn{4}{|c|}{ Silica } \\
\hline Borofloat \\
\hline \multicolumn{4}{|c}{}
\end{tabular}

Fig 3. 4 Model for simulation in OPTIBPM 7.0

The power at the ends of the waveguides is recorded and simulations are performed for a series of refractive indices of the analyte. Based on the power observed at the ends during measurement these can be solved using BPM simulations to get the effective refractive index of the material present on the biolayer.

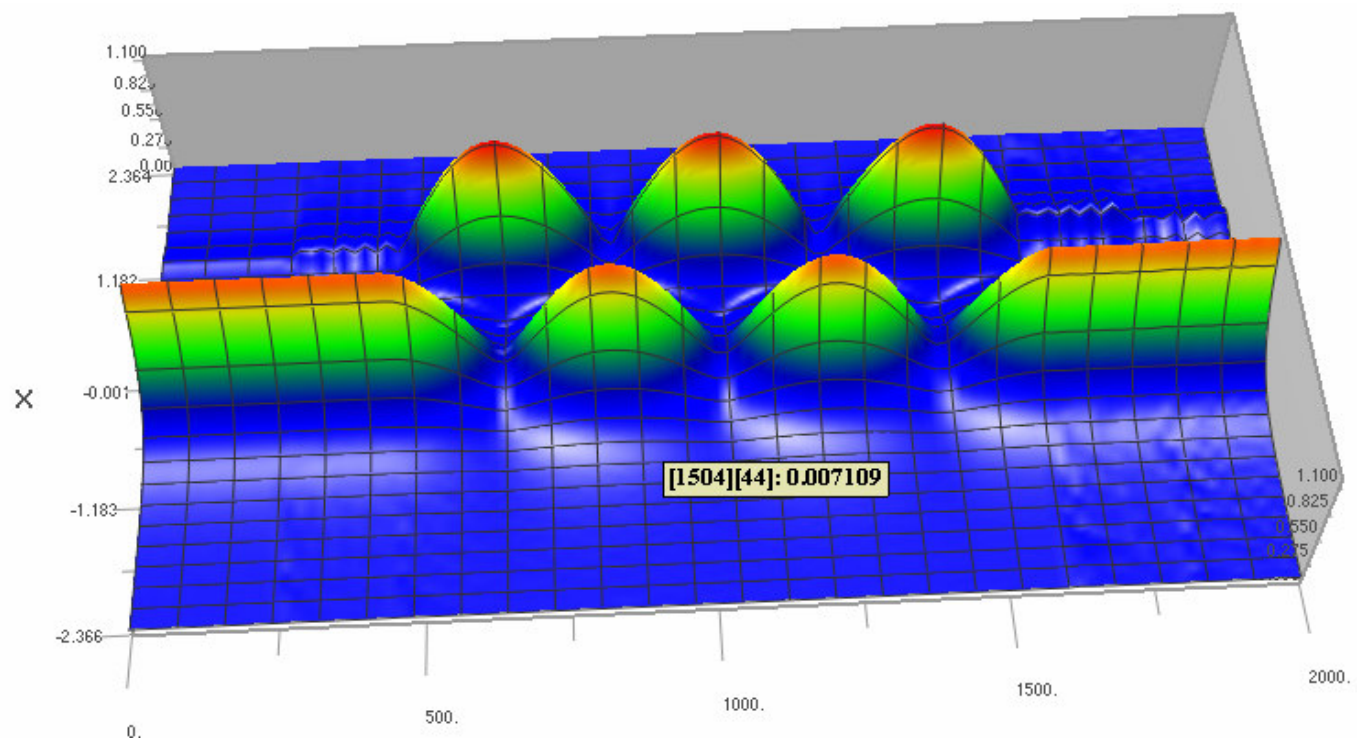

Fig 3. 5Field Propagation in designed structure

\subsection{PMMA Patterning:}

PMMA (Polymethyl Methacrylate) is a polymer material and is generally used as a protective coating for wafer thinning, bonding adhesive and sacrificial layer. In our device a channel should be present on the waveguide surface for the bioagent flow. Also dust or unwanted particles on the waveguide surface might create unnecessary power transfer and hence waveguide has to be protected by cladding leaving windows for power observation, prism coupling and microfluidic channels. A protective layer of PMMA is spun on the top waveguide and channels are patterned on it enabling bioagent flow and 
coupling of light with the prism coupler. Fig 3.6 shows the final structure after PMMA patterning. PMMA etching is done using Reactive Ion Etching by a shadow mask using oxygen plasma. Based on the Ellipsometer readings of alumina film thicknesses before PMMA coating and after PMMA etching it has been found that oxygen plasma wouldn't etch the alumina film. Hence oxygen plasma has been chosen to etch the PMMA. The etching is performed by RF plasma of power $200 \mathrm{~W}$ at $30 \mathrm{sccm}$ of oxygen flow and chamber pressure at 10 millitorr. The etch rate was around $100 \mathrm{~nm}$ per minute. Fig 3.6 shows a patterned PMMA on Borofloat stack with all the windows etched. A mechanical apparatus as shown in Fig 3.7a\&b was initially employed for flow cell experiments. Due to its bulk structure it was very difficult to couple light into the modes and even though the light couples the stress produced by the heavy steel apparatus on the prism coupling spot for the films was high and hence the streak often vanishes.

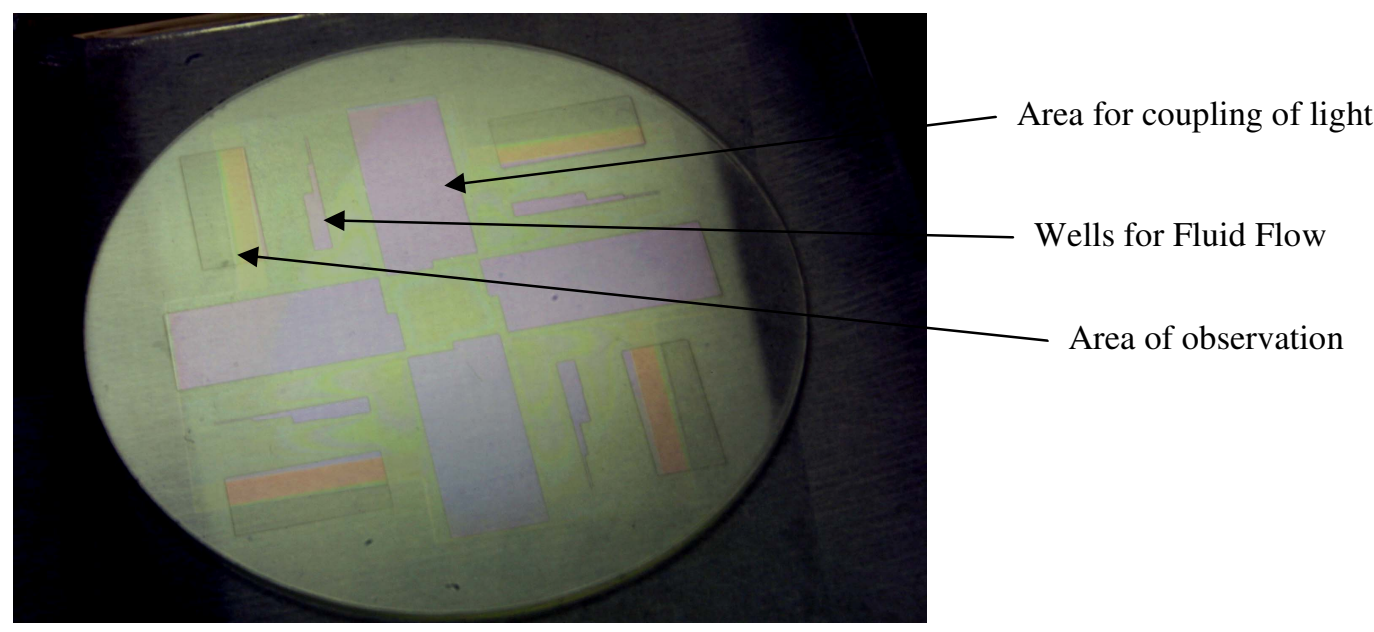

Fig 3. 6 Final PMMA pattern on Borofloat Substrate [18]

Due to the above complexities a new arrangement for providing channels on waveguides has been developed. PDMS channels have been fabricated using silicon master and are bonded on the waveguides. Capillary tubes along with a syringe pump were used for pumping the analyte solution. The details of this process are discussed in the next section. 


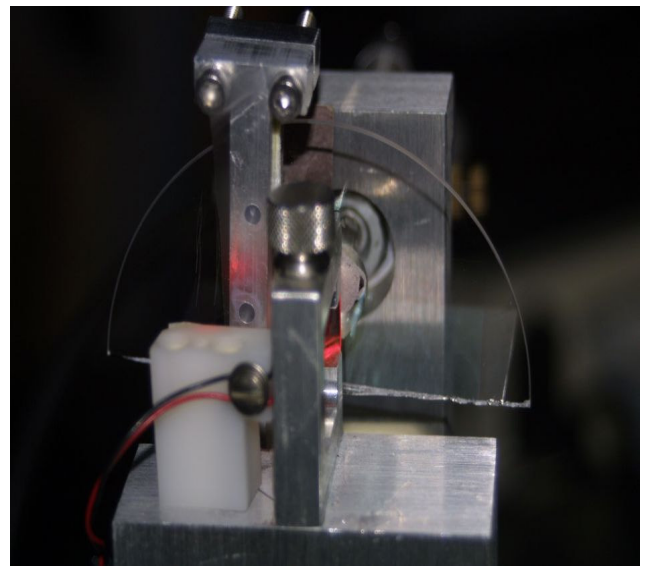

Fig 3. 7a Flow cell arrangement

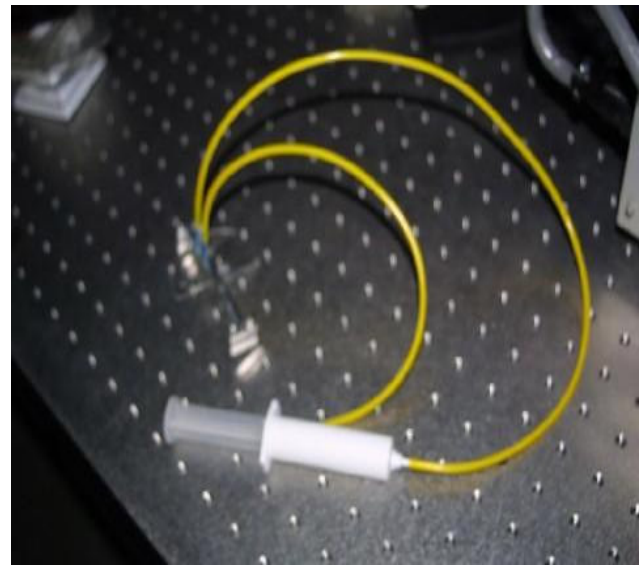

Fig 3.7b Syringe for the analyte flow

\subsection{PDMS channels and Silicon Master Fabrication:}

Photo masks were generated using photo plotting process with a resolution of 8000 dpi at WVU Printing Services. The mask designs were created in "Freehand". This mask has been used to fabricate silicon master which in turn is employed in making PDMS molds. A negative topographical structure has to be patterned on silicon wafer. Fig 3.7c shows a general picture of PDMS mold. Four different channels have been patterned on the silicon wafers each of different thickness. Each channel has a scale associated with it to calculate-the hydrodynamic flow. The distance between the scale and the channel is around $1 \mathrm{~mm}$. The mask is printed using a dot-matrix printer. NanoSU8-25, negative photo resist, is used to lay the patterns on the Silicon master. Fig 3.9 is a general silicon master fabricated using the above process.

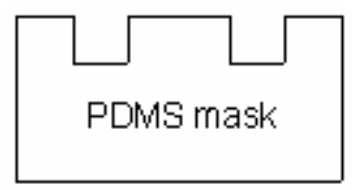

Fig 3.7c Front view of a PDMS mask with two channels embedded

\subsubsection{NanoSU8-25}

Nano SU8 is a high contrast epoxy based negative photo resist. It is transparent above $360 \mathrm{~nm}$ and is a good option for features requiring good vertical sidewalls and thick 
films. This is best suited for permanent applications like masters for the molds where it is imaged, cured and left in place.

A general process for fabrication is spincoat, softbake, expose, post exposure bake and develop. Post exposure bake is the critical step for the whole process as cross linking takes place at this time. Cross linking takes place in two steps.

1) Formation of a strong acid during the exposure process

2) Acid initiated, thermal driven epoxy cross-linking during the post exposure bake The lithography process is as follows:

- A 3 inch silicon wafer is cleaned in acetone and methanol, 5 min each and blown dry in nitrogen.

- The wafer is then subjected to Hydrofluoric acid to remove the native oxide if any and then heated in the oven at $120^{\circ} \mathrm{C}$ for 10 min to dehydrate the surface.

- Photoresist (NanoSU825) is applied at the center of the wafer and is spun on a spinner at $950 \mathrm{rpm}$ for 90 seconds with an acceleration of $100 \mathrm{rpm} / \mathrm{s}$ for the initial 10 seconds. A thickness of 40 micron film is obtained at the end of this process. The spin - speed curves are shown in the Fig 3.8.

- It is then baked on hotplate at $90^{\circ} \mathrm{C}$ for $22 \mathrm{~min}$ and cooled for 10 minutes.

- The wafer is aligned with the mask and exposed to UV light at $320 \mathrm{~nm}$ for 20 seconds as it should penetrate 40 micron thick resist. Exposure times would vary depending on the thickness of the resist.

Note: The mask is aligned with the wafer and is secured by placing a 1/16 inch quartz plate on it.

- Post exposure bake is performed at $60^{\circ} \mathrm{C}$ for $5 \mathrm{~min}$ and at $90^{\circ} \mathrm{C}$ for $15 \mathrm{~min}$ on a hotplate. The sample is left for cooling for $5 \mathrm{~min}$ after this process.

- Photo resist is developed by immersing the sample for $3 \mathrm{~min}$ in SU8-developer. Large beakers are recommended for this process.

- Subsequent rinsing is performed in iso-propanol or acetone to remove the residues of the developer if any. 


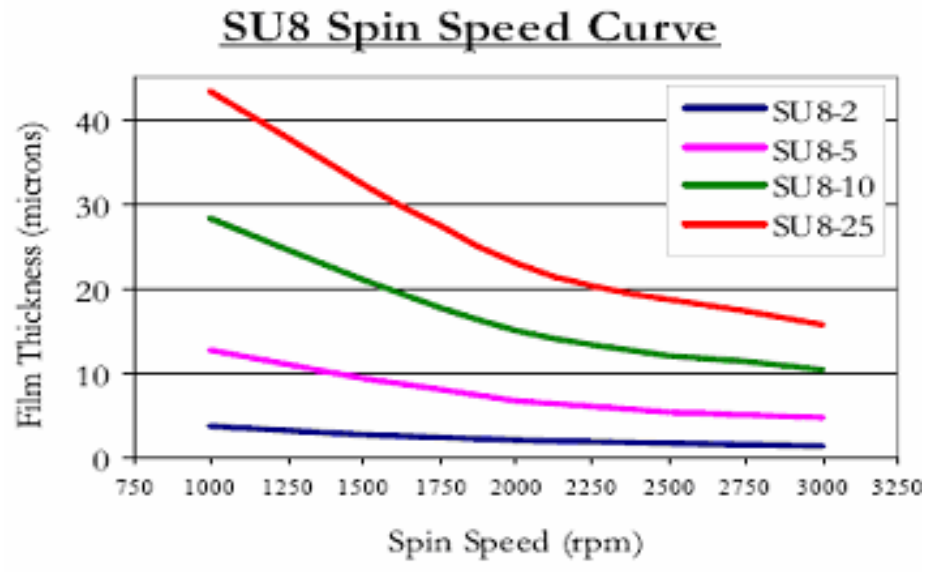

Fig 3. 8 Spin Speed Curves for NanoSU8 [32]

Thin silicon wafers tend to break during this peeling process. Thick silicon wafers (600-800nm) were chosen for this process as the wafers have to withstand the physical force of peeling created when the PDMS mask is removed after curing. A silicon master is shown in Fig 3.9

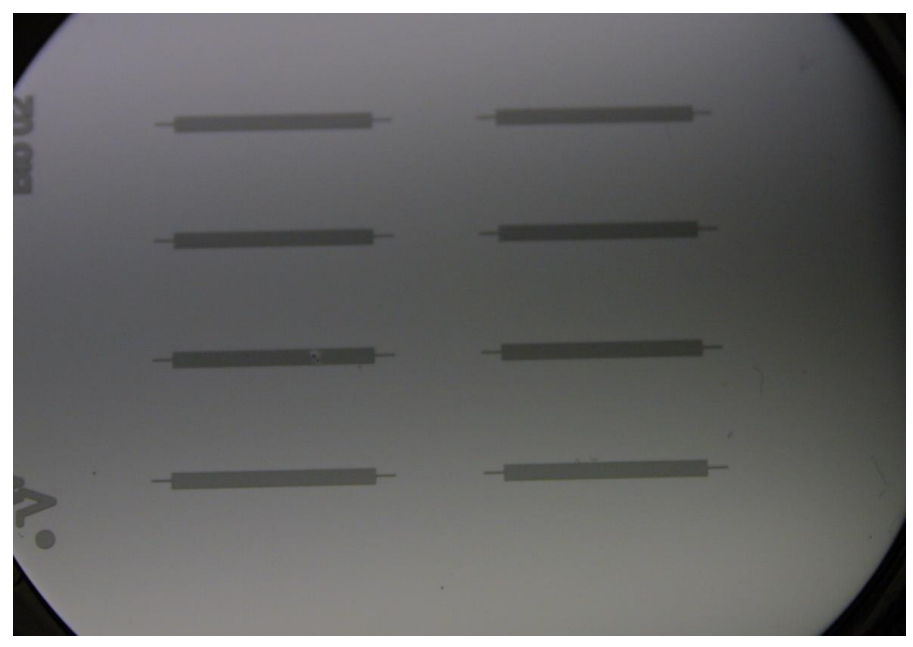

Fig 3. 9 Silicon master fabricated using Nano SU8-25

\subsubsection{PDMS (poly dimethyl siloxane):}

The fabrication of microfluidic channels can be done by different types of plastics like polycarbonate, polyester, polystyrene, polyethylene terephthalate glycol, polymethyl methacrylate (PMMA) and polyolefins but the most popular material that is used is polydimethylsiloxane (PDMS) [33]. It is inexpensive, easily molded from the prepolymer, has good electrical resistivity, good optical transmission properties and adequate thermal conductivity. It also has few disadvantages like low electro osmotic 
flow (has quarter to threefourth compared to that of glass), hydrophobicity (difficult to wet the PDMS channels) and importantly hydrophobic analytes gets adsorbed on the PDMS channels. This significantly limits the analytes and hence results in lower separation efficiency. To overcome the above problem the PDMS channel surface has to be made inert.

PDMS chip containing microfluidic channels was fabricated using a Sylgard ${ }^{\circledR} 184$ Silicone Elastomer Kit purchased from Dow Corning Corporation (Midland, MI). Elastomer base and curing agent was mixed at a weight ratio of 10:1 by stirring for $40-$ 60 minutes. This mixture has been degassed for $40-60$ minutes with a vacuum pump to remove air bubbles generated during stirring action. It was then applied on the surface of silicon master and cured overnight in the oven at $70-90{ }^{\circ} \mathrm{C}$. The apparatus used for making the chip is shown in the fig3.10.

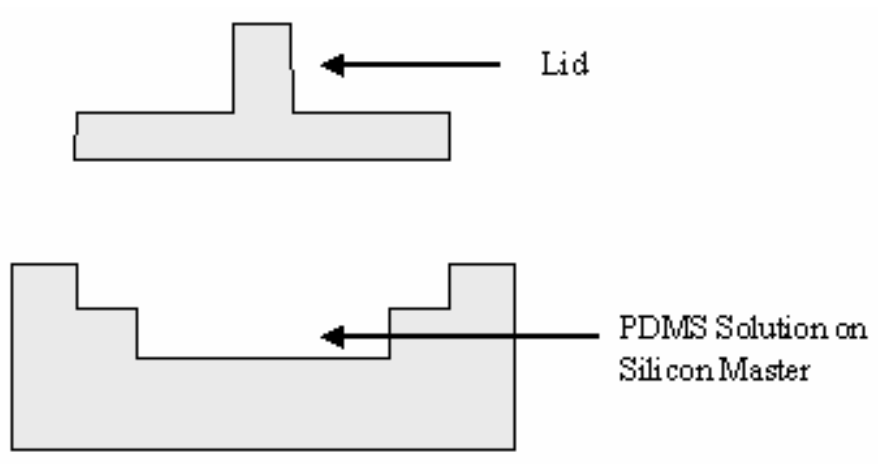

Fig 3. 10 Design for PDMS mask fabrication

The surface properties of the PDMS are varied by using a coating layer. This is a sol-gel process to form nanometer sized silica particles throughout polymerized PDMS surface. An alkoxysilane precursor such as tetraethyl orthosilicate (TEOS) is hydrolyzed and then condensed on the PDMS surface [34]. An acid or base is generally used for the condensation reaction. TEOS solution was made by a volumetric ratio of 2:1:1:1 with TEOS, ethanol, DI water, and $5 \mathrm{mM} \mathrm{HCl}$. TEOS coating on the surface of PDMS channel was made by continuously flowing the solution for $30 \sim 60$ minutes.

After this process the PDMS surface would be coated with permanent free silanol groups and thus would increase Electro osmotic flow and wettability. The 
absorption of the hydrophobic materials to the PDMS surface is also reduced. This also would allow sealing of PDMS to another PDMS if necessary.

\subsubsection{PDMS binding to the Alumina Surface:}

Though PDMS sticks to the alumina surface laterally, there would be wavelength comparable air cavities which results in high scattering loss. The light streak that enters into the PDMS region loses its intensity by the time it leaves the PDMS region and hence an efficient way of binding PDMS to the alumina surface was explored. There were many techniques to bind glass to glass and silica to silica using PDMS but no established technique, to our knowledge, is present for binding PDMS to alumina surface. Oxygen Plasma binding is adopted to bind the PDMS chip to the alumina surface as binding glass to PDMS and getting irreversible seals is well known.

The changes in surface chemistry have been well studied by different research groups. Most of them conclude that PDMS material in general has chains of $-\mathrm{O}-\mathrm{Si}\left(\mathrm{CH}_{3}\right)_{2}$ -, which on exposure of oxygen plasma develops silanol groups $(-\mathrm{OH})$ at the place of methyl groups $\left(-\mathrm{CH}_{3}\right)$ [35]. The oxidation of the surface layer (in this alumina or glass) increases the hydroxyl groups and this leads to the formation of strong intermolecular bonds. The silanol groups condense with those of similar surface when brought into contact. The PDMS to glass surface produce Si-O-Si bond while we expect an Al-O-Si bond between PDMS and alumina interface. This covalent bond would form the basis for the tight irreversible seal between the layers. An optimum point has to be reached to get an efficient bond between the layers.

Process for PDMS binding to Alumina Surface:

- Clean the alumina substrate using a series of polar and non polar solvents consecutively 5-10 min each.

- Bake the Alumina sample to dehydrate the surface.

- Sonicate the PDMS chip in Isopropanol for 30min to remove the surface impurities if any.

- Bake the PDMS chip in oven for $15 \mathrm{~min}$ at $100 \mathrm{C}$ 
- Place the samples in the plasma cleaner chamber taking care that the surfaces to be bonded are faced to the plasma.

- Expose the samples for a span of minute.

- Hydrolyze the surfaces (formation of $\mathrm{OH}$ bonds) by dipping them in de-ionized water for $10 \mathrm{~min}$.

- Align the sample (Alumina and PDMS) and bake it for three hours or more at 100C 


\section{CHAPTER 4: Experimental Analysis}

This chapter details the comparative results obtained between measured and simulated beta values for single and multi-layer films. The beta values are measured from prism coupler. Optical waveguide loss dependence on ion beam parameters like oxygen flow rate and ion drive current is performed. Refractive index variation of the films when annealed and water soaked is also discussed. A sensitivity analysis has been performed to observe variation in parameters like power transfer and coupling length between the waveguides. Top waveguide thickness and index were used to perform the analysis. We would also discuss the methodology adopted to conduct flow cell experiments in the micro fluidic channels and finally we would discuss the problems we are facing in modeling the structures using ellipsometry.

\subsection{Beta Values (Measured Vs Simulated)}

Plane waves interfere as they propagate to form a repeating pattern when they are traveling in a waveguide. The propagation constant or Beta of a plane wave is defined as a periodic frequency at which the wave repeats. It is defined as Eq 4.1.

$$
\beta=k n_{\text {eff }}=\frac{2 \pi}{\lambda} n_{\text {eff }}
$$

The propagation constant depends upon the modal angle of the waveguide, refractive index of the waveguide and the wavelength of propagating light. The thickness and the index of refraction of the waveguide can be verified indirectly by measuring the propagation constants of the waves as the modal angle depends on these parameters. The prism coupler was used to calculate the beta values and was compared with the values obtained from ' $2 \mathrm{~d}$ modesolver'. The thicknesses and indices for mode solver were obtained from the ellipsometer. Table

4.1a shows the beta values for Al-Si-Al films grown on Borofloat substrate while Table $4.1 \mathrm{~b}$ shows the beta values of single layer alumina films on Borofloat substrate. Simulated beta values were obtained from ' $2 \mathrm{~d}$ Modesolver' and experimental beta values 
were calculated from Prism coupler measurements. We observed that the beta values obtained from the prism coupler match with those obtained from simulations.

\begin{tabular}{|l|l|l|l|l|}
\hline Sample & Beta0(Sim) & Beta1(Sim) & Beta0(PC) & Beta1(PC) \\
\hline B502AOA & 14.97646 & 14.7836 & 14.9729 & 14.77604 \\
\hline B503AOA & 14.97687 & 14.84931 & 14.99676 & 14.82376 \\
\hline B504AOA & 15.02758 & 14.9036 & 15.03653 & 14.85856 \\
\hline B501AOA & 14.94505 & 14.84045 & 15.02261 & 14.89535 \\
\hline B500AOA & 14.99735 & 14.96087 & 15.0405 & 14.78399 \\
\hline
\end{tabular}

Table 4.1a Beta Values for stack that has two modes

\begin{tabular}{|l|l|l|}
\hline Sample & Beta0(Sim) & Beta0(PC) \\
\hline B422A & 14.78797 & 14.7511 \\
\hline B428A & 14.783 & 14.9281 \\
\hline B437A & 14.8436 & 14.8743 \\
\hline
\end{tabular}

Table: 4.1b Beta Values for single layer alumina films

\subsection{Waveguide loss variation with Ion Beam Parameters}

The film quality depends on many parameters during Ion Assisted e-beam deposition process. The primary parameters effecting film quality are ion flux and drive voltage [36]. Drive voltage is set by the ion drive current and ion flux is dependent on the inlet gas flow rate. Though drive voltage is set by the ion drive current there are few other parameters that influence film quality like substrate heat and mean collision path. Details about the influence of these parameters can be found in [36]. Films were grown at varying ion drive currents and oxygen flow rates. The optical film quality was assessed in each case. Optical film quality refers to the scattered streak quality and optical propagation loss. Fig 4.1 shows the optical loss variation with ion drive current for the films grown at constant oxygen flow rate of $18 \mathrm{sccm}$. We observed that the optical propagation loss increased with ion drive current. 
The losses are calculated using optical scattering measuring technique $[37,38]$ where the optical image of the guiding streak is captured using a CCD camera and curve fitting analysis in Matlab is employed to fit the column intensities. The slope of the column intensities would give us the optical loss of the film. Details of this process are mentioned in Appendix B.

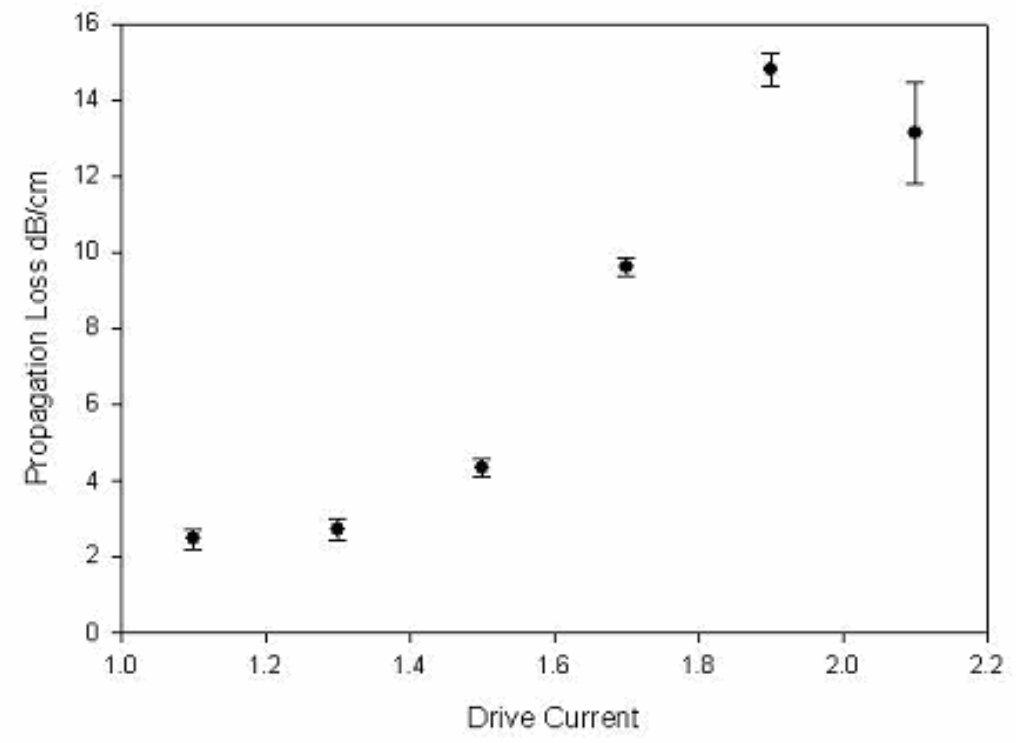

Fig4. 1 Optical loss variation with ion drive current

The streaks corresponding to the films grown at ion drive currents of $1.3 \mathrm{~A}$ and 1.9A are shown in Fig 4.2a and Fig 4.2b. It was observed that films deposited at low ion drive current yield better guiding streaks. The streak quality was found to decline with increase in the ion drive current. The reasons for this behavior were not yet established. We believe that the density variation of the films is greater at higher ion drive currents. 


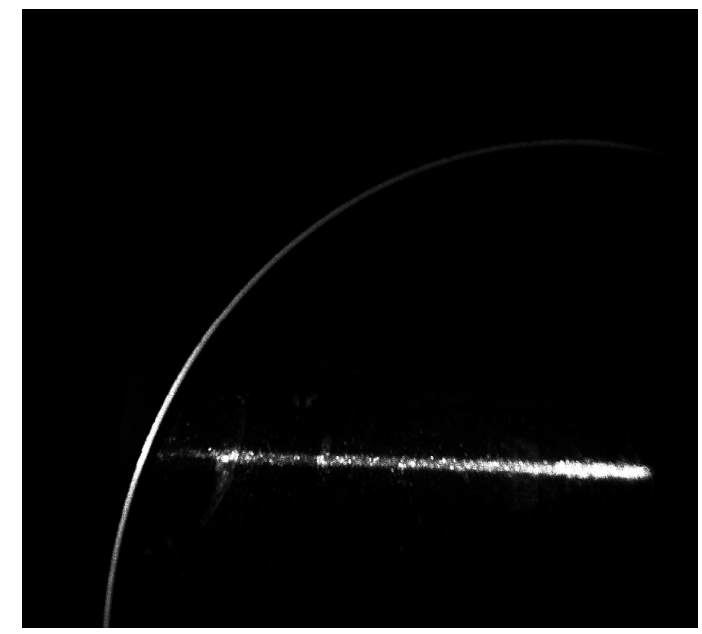

Fig4. $2 a$ Streak at $I d=1.3 A$

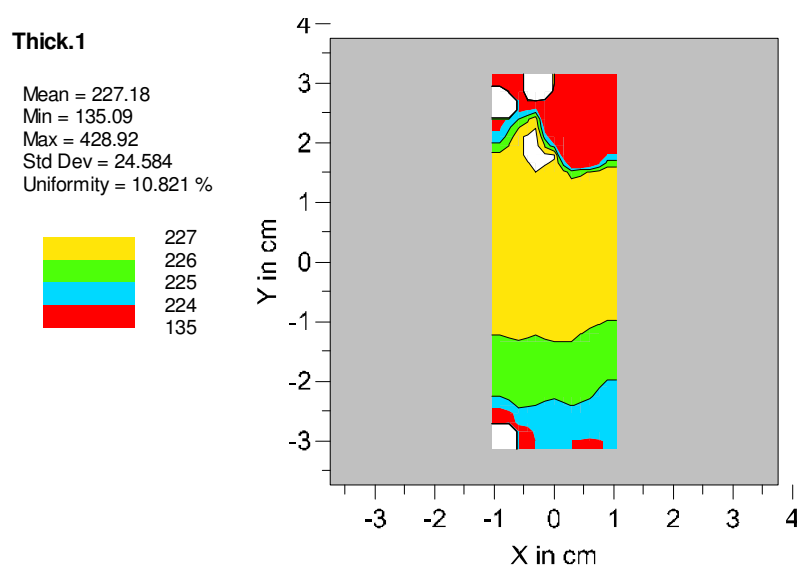

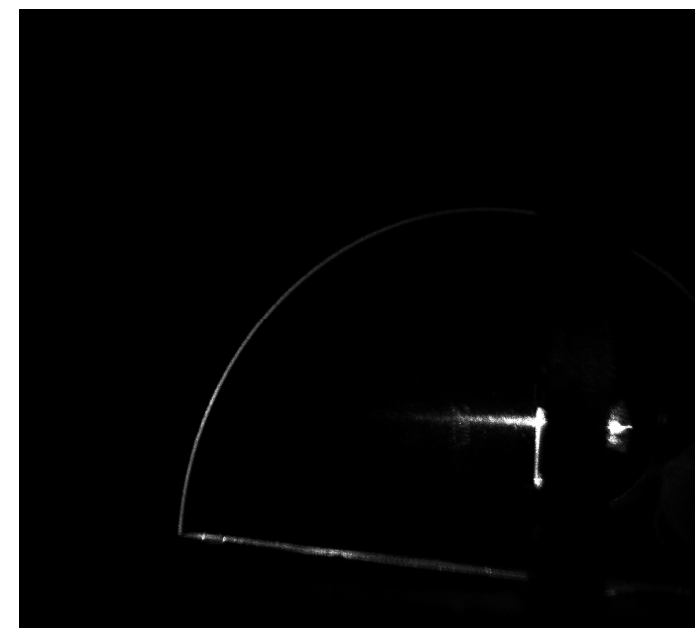

Fig 4.2b Streak at $I d=1.9 A$

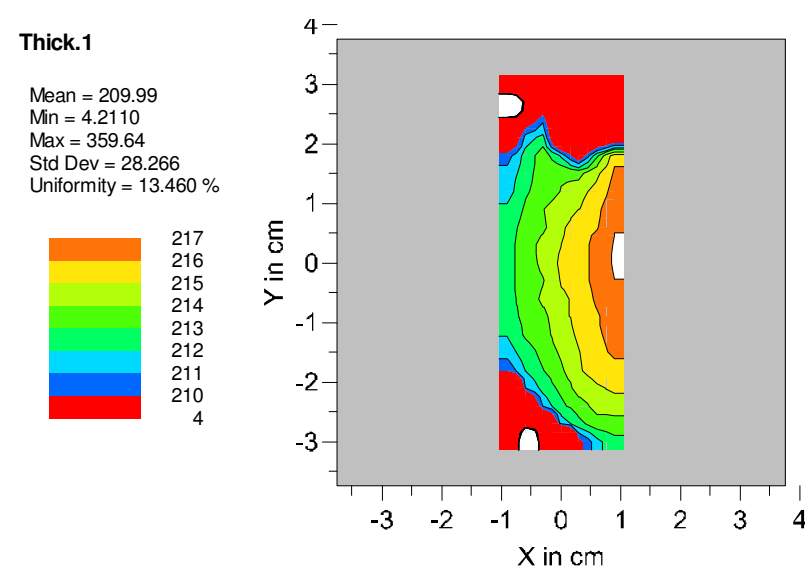

Fig4. 3Thickness Variation of (a) High loss film (b) Low loss Film

Fig 4.3a\&b shows the thickness variation obtained using ellipsometer. There was a maximum surface roughness of $2 \mathrm{~nm}$ on a 3inch wafer which is with in the operation limits of the biosensor. Fig 4.3c\&d shows the index distribution on the whole surface. The Cauchy model is a general technique to model the dielectric films and $A_{n}$ can be approximated to the index of refraction at larger wavelength. The index is modeled as [39]

$$
n(\lambda)=A_{n}+\frac{B}{\lambda^{2}}+\frac{C}{\lambda^{4}}
$$


Ellipsometer results indicate that there is no significant difference between the surface profiles and index distributions of high loss films and low loss films. If the model used in Ellipsometer to measure the thickness was assumed to be accurate then the actual reasons for this behavior are yet to be known.
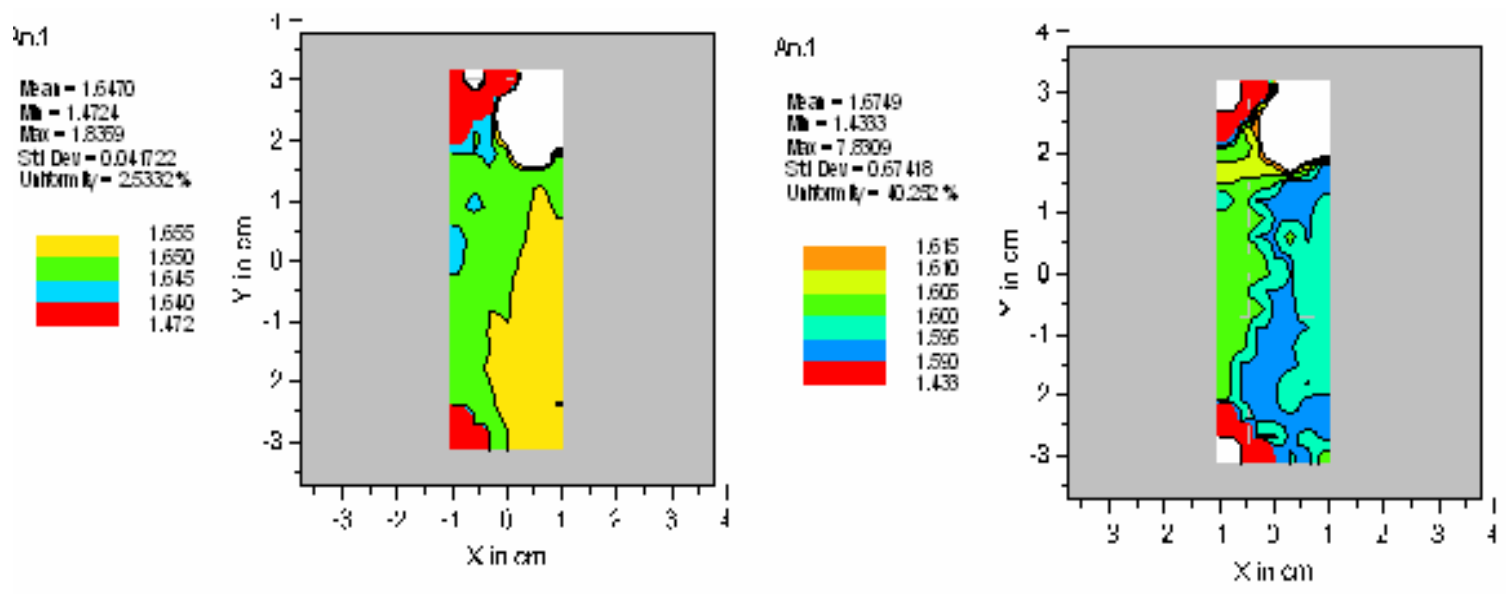

Fig 4.3 Refractive Index $\left(A_{n}\right)$ Variation (c) High loss film (d) Low loss film

Fig 4.4 shows the refractive index variation of alumina films as a function of ion drive current and oxygen flow rate. Alumina films were grown for a set of ion drive currents and oxygen flow rates. It was observed that refractive index increases with ion drive current and decreases with oxygen flow rate. Fig 4.1 shows that films at higher ion drive currents have higher losses. Also, films deposited at lower ion drive currents were found to have unstable lower refractive indices that vary with temperature and water soaking. Hence an intermediate drive current of $1.3 \mathrm{~A}$ has been chosen for waveguide depositions. These issues will be discussed in more detail in a subsequent thesis [40]. 


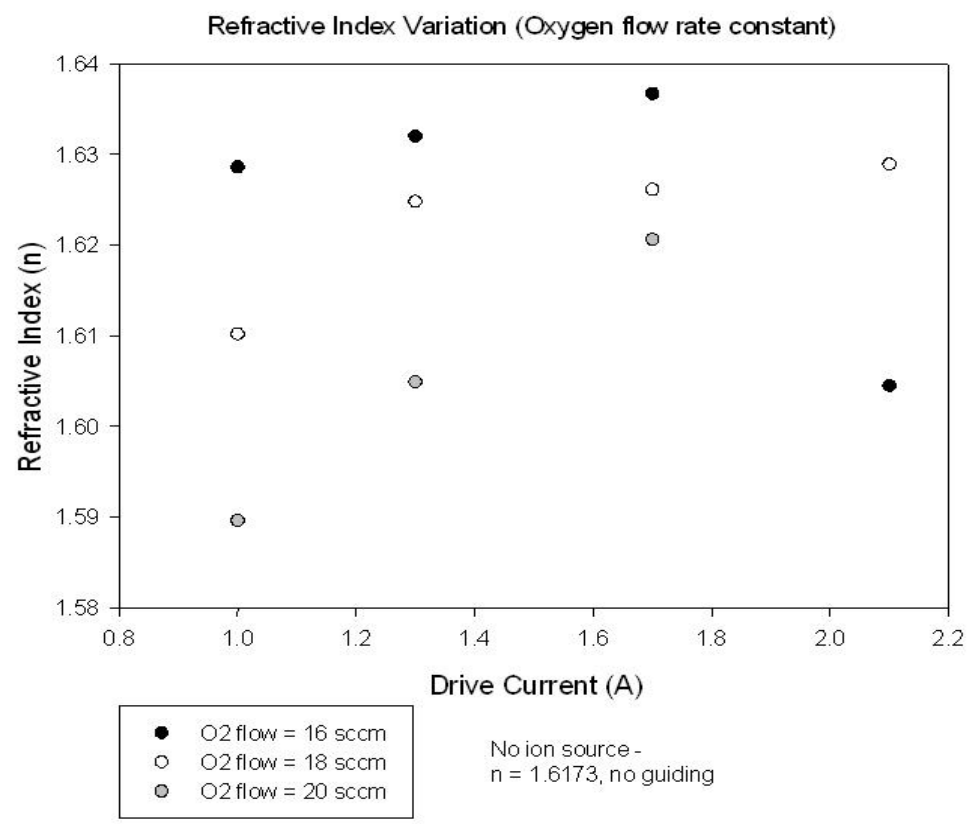

Fig4. 4 Index Variation with Drive Current

The next parameter of interest is the oxygen flow rate. The greater the oxygen flow rate the higher is the ion flux generated inside the chamber. Higher oxygen flow rates would increase the amount of gas molecules present in the chamber and thus

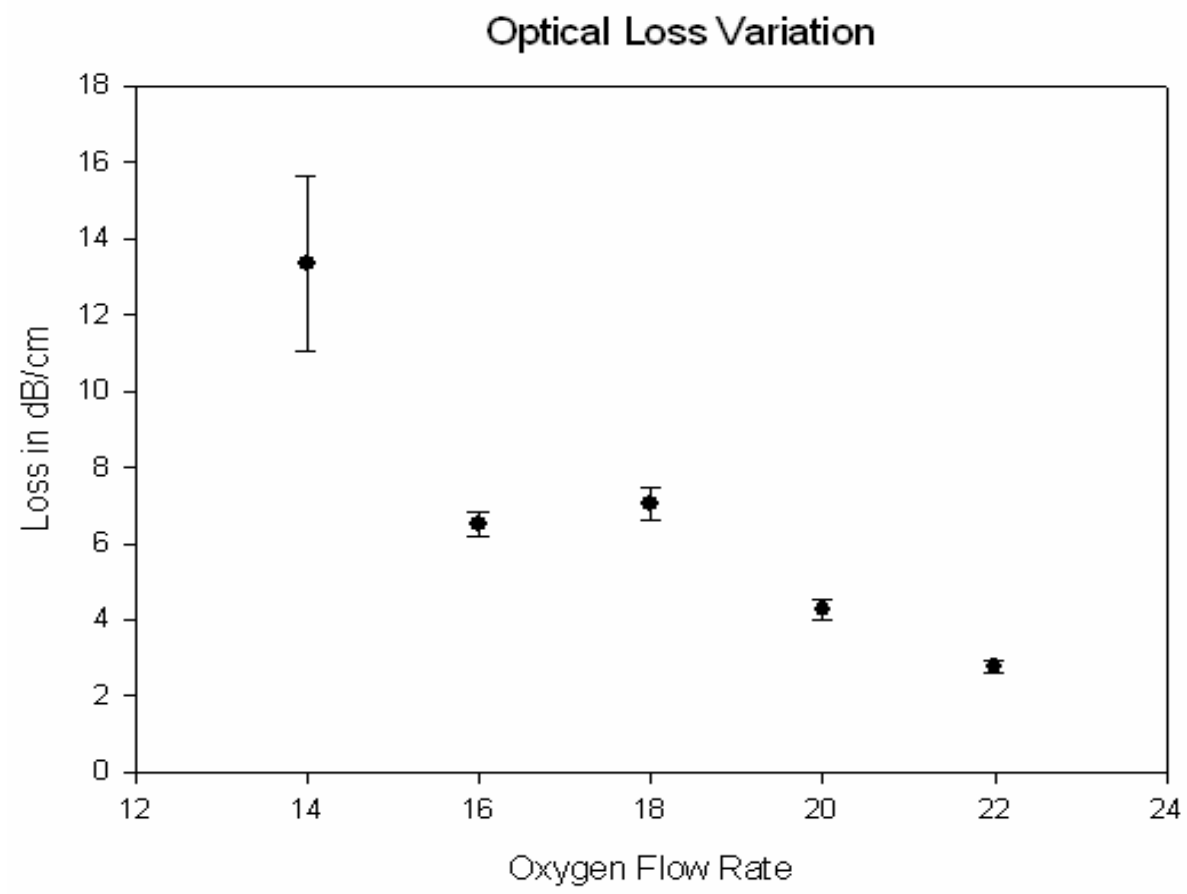

Fig4. 5 Optical Loss Variation with Oxygen Flow rate 
decrease the mean free path. Increase in mean free path would increase the number of collisions and hence would result in unfavorable depositions. On the contrary, depositions with lower flow rates do not have enough number of gas molecules to create and sustain plasma. Fig 4.5 shows that increase in the oxygen flow rate decreases the optical propagation loss at a fixed ion drive current. Fig 4.6a\&b shows two films deposited at ion drive current of $1.3 \mathrm{~A}$ and oxygen flow rate of $22 \mathrm{sccm}$ and $14 \mathrm{sccm}$ respectively. We concluded that films deposited at higher oxygen flow rates gives good streaks. From Fig 4.4 it was observed that higher oxygen flow rate would give lower index of refraction. For lower losses and good refractive index an intermediate oxygen flow rate, $18 \mathrm{sccm}$, has been chosen for alumina waveguide depositions. Fig 4.10 shows the combined plot of loss variation with oxygen flow rate and ion drive current. The $\mathrm{x}-$ axis represents the ion drive current, y-axis represents the propagation loss and the values written at each point are the oxygen flow rates. We concluded that the losses increase with ion drive current and decrease with flow rate.

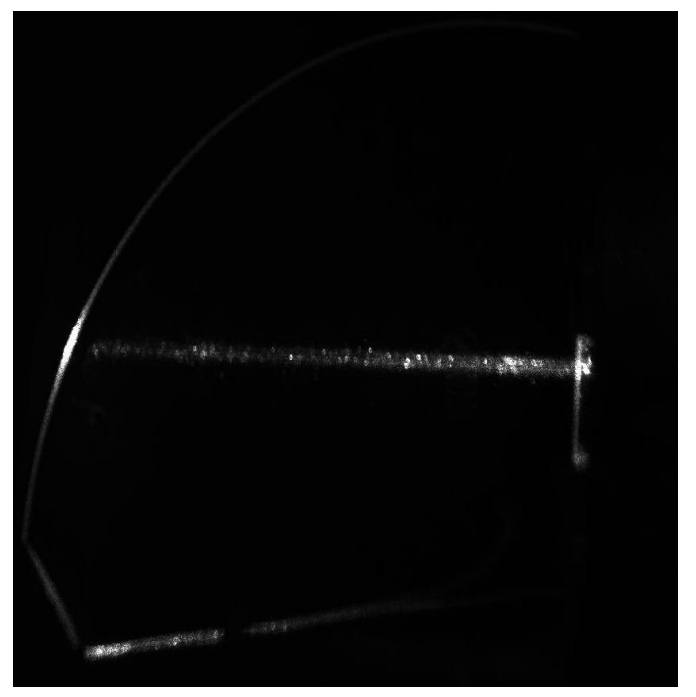

Fig4. $6 a \mathrm{Id}=1.3 \mathrm{~A}$ and $\mathrm{O} 2=22 \mathrm{sccm}$

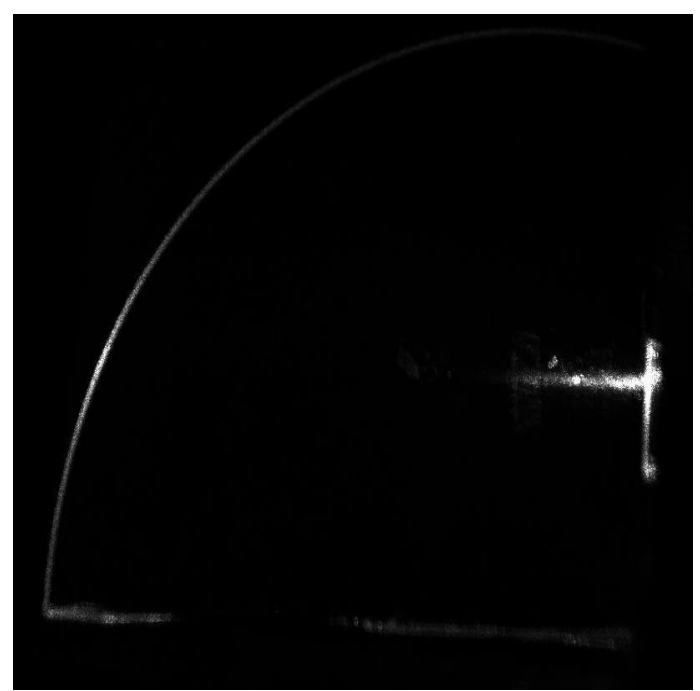

Fig $4.6 b \mathrm{Id}=1.3 \mathrm{~A}$ and $\mathrm{O} 2=14 \mathrm{sccm}$

Fig 4.7 shows the thickness variation of alumina waveguide with water soaking time. Three samples deposited at different oxygen flow rates and at fixed ion drive current of 1.3A have been chosen for analysis. We observed that the thickness variation is higher for films grown at higher oxygen flow rates. This variation though not intended is 
very less at lower oxygen flow rates. Fig 4.8 shows the refractive index variation of the samples with soaking time.

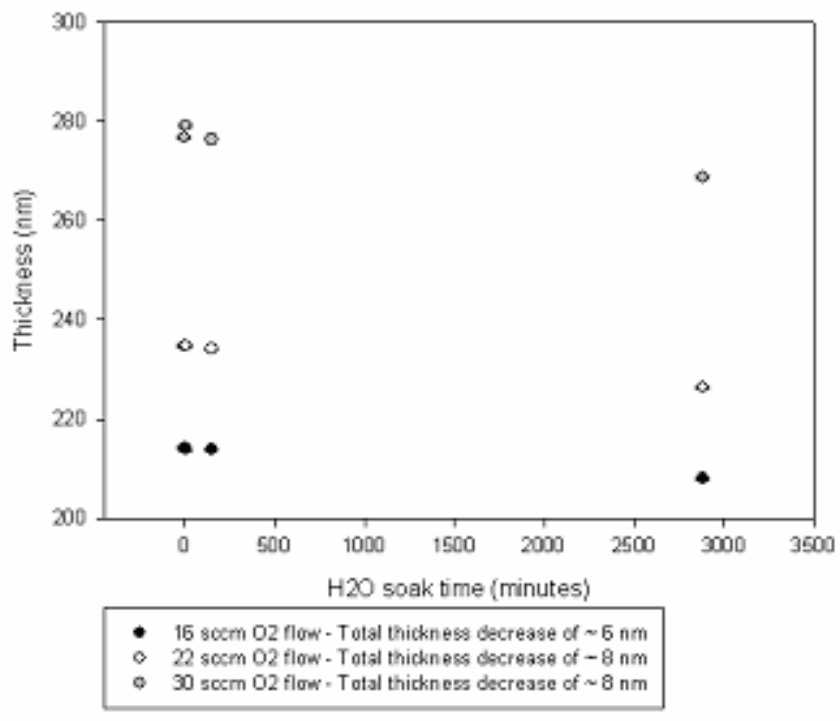

Fig4. 7 Thickness change with soaking time

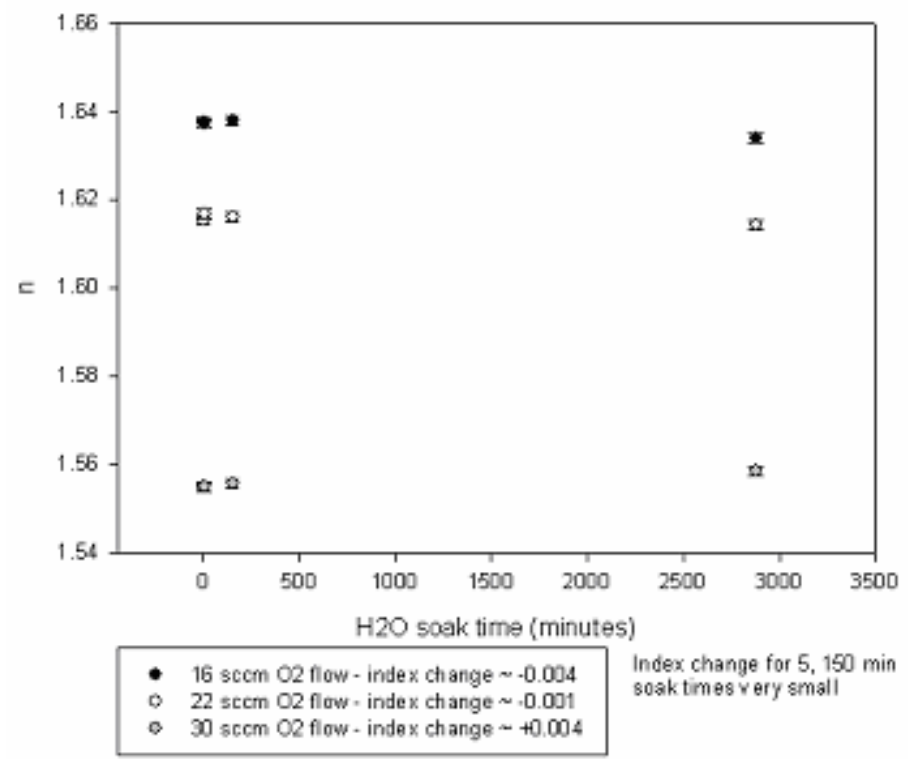

Fig4. 8 Refractive Index Variation with soaking time

The index of refraction also changed with soak time though the change is very less. The samples were annealed to check the temperature stability. Samples were heated at $600^{\circ} \mathrm{C}$ for $5 \mathrm{~min}$ and were measured using ellipsometer. Fig 4.7 shows the index variation with temperature. It can be seen that the index decreases significantly for higher 
oxygen flow rates while appreciable change is not present for samples grown at lower oxygen flow rates.

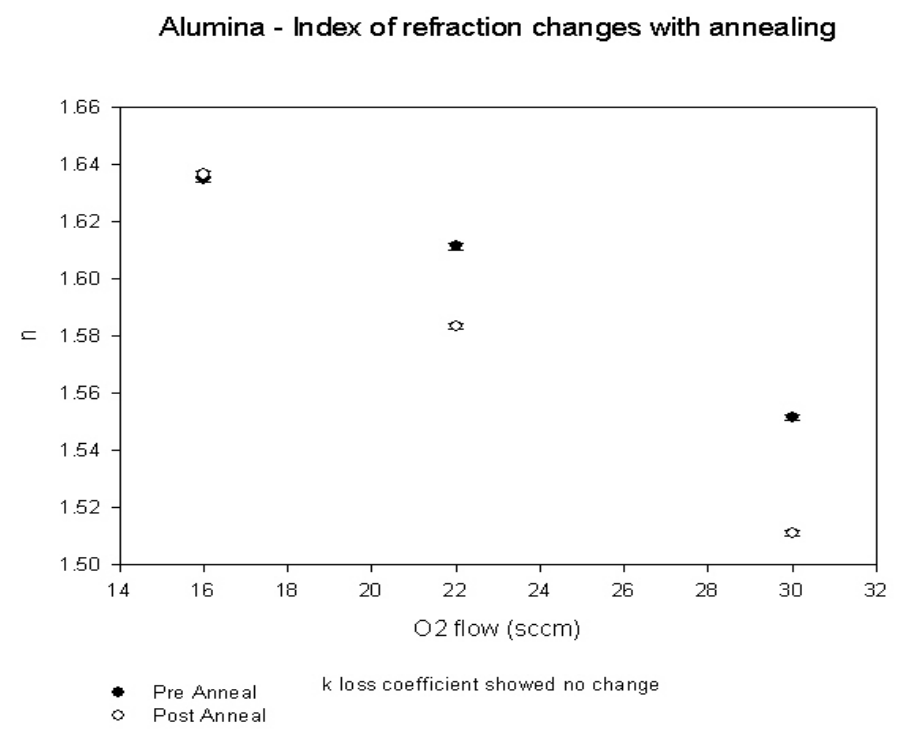

Fig4. 9 Refractive index variation with annealing

Alumina films grown at lower oxygen flow rates tend to have stable refractive indices and hence oxygen flow rate of $18 \mathrm{sccm}$ has been chosen for waveguide depositions.

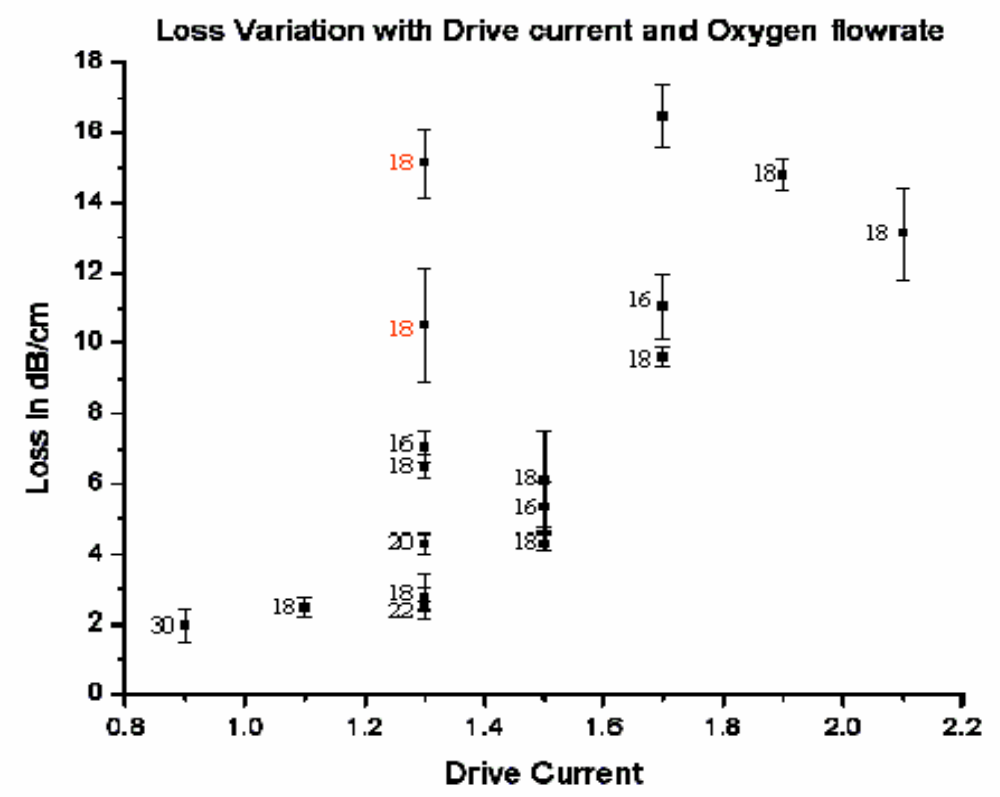

Fig4. 10 Loss variation with ion drive current and oxygen flowrate 


\subsection{Sensitivity Analysis:}

In spite of many measures taken to control the index and thickness of the waveguides we will still end up with a variation in the final parameters of the waveguide from the required thicknesses and indices due to fabrication difficulties and device control limitations. Changes can also arise due to moisture exposure as discussed previously. Two methods have been developed for fabricating the desired structure. The first and usual fabrication process starts with depositing bottom two layers of alumina and silica at a time using first mask on Borofloat substrate and then the top waveguide is deposited using second mask. Fig 4.11a \& b represents the first and second mask during fabrication the masks are present to discern the fabricated waveguides. This method requires vacuum break after depositing bottom alumina and silica films. So control over the top layer is often different with the bottom layers' control. This results in wide variation of thicknesses and indices from the desired model for the top waveguide.

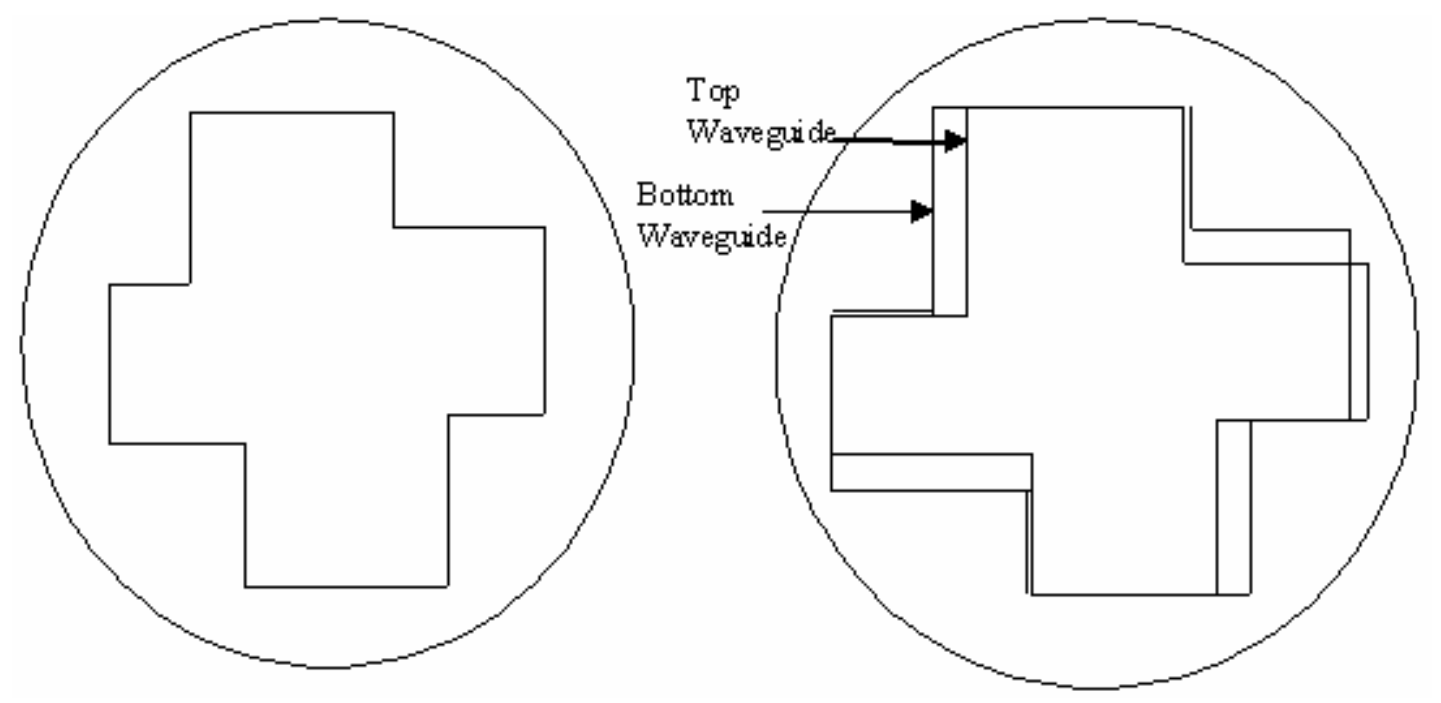

Fig4. 11 (a) First Mask (b) Second Mask

Bottom layer depositions' thicknesses and indices are measured using ellipsometer and offsets from the designed model are incorporated to design a new model with variations in the top waveguide to compensate the offset. The top waveguide after deposition might still have variations from the newly designed model and hence its effect on the output power has to be studied. Similarly ambient conditions might result in change of film properties to a little extent due to relaxation of the films. So an analysis 
has been made to study the effect of refractive index and thickness change on coupling length and power transfer between the waveguides. Coupling length and power transfer variation with the top waveguides' index and thickness has been calculated using $2 \mathrm{D}$ mode solver and OPTIBPM 7.0. 2D mode solver has been used to evaluate the modal indices of the structure. The top waveguide thickness and refractive index has been changed from $180 \mathrm{~nm}$ to $205 \mathrm{~nm}$ and 1.626 to 1.692 respectively. The power transfer variation and coupling length variation for the desired structure are calculated by varying thickness and refractive index of the top waveguide and are shown in fig 4.1 and fig 4.2. We observe that both the graphs show similar trend.

\section{Power Transfer between the waveguides}

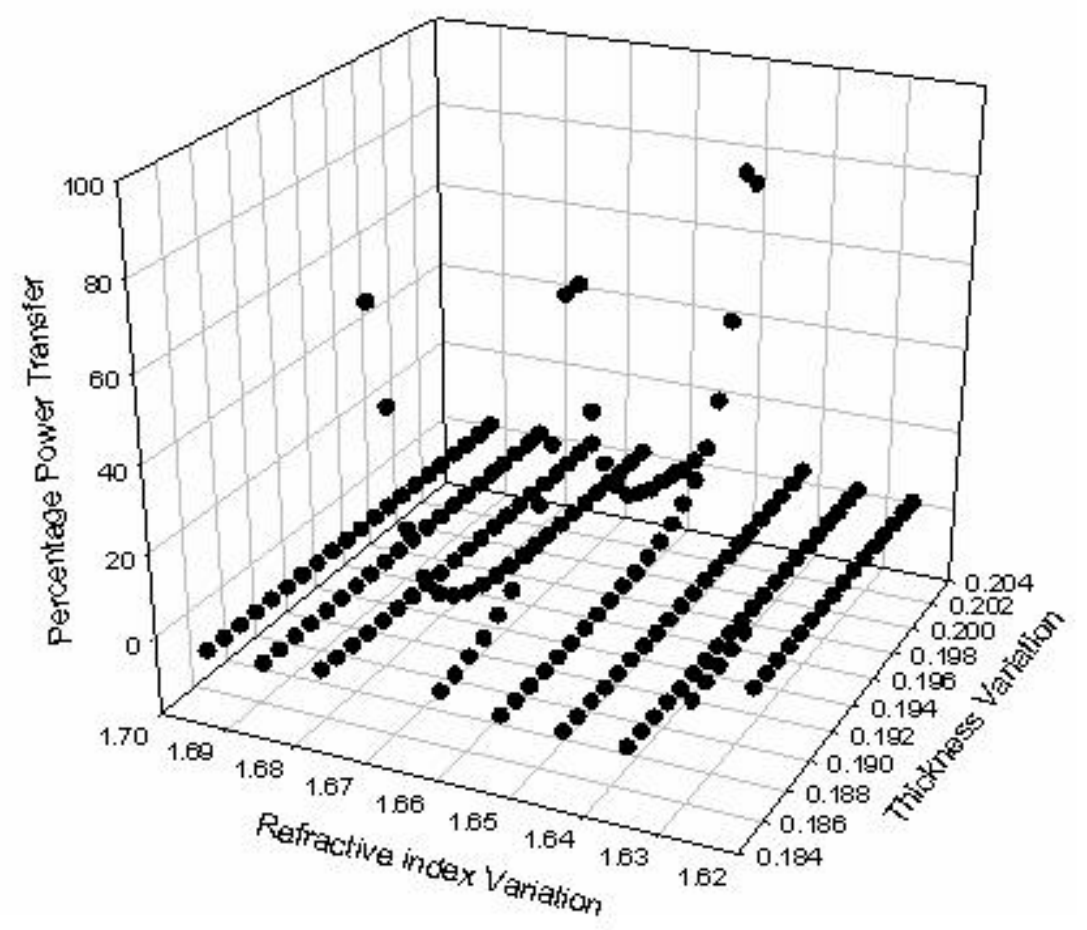

Fig4. 12(a) Power Transfer Variation with Top Waveguide Thickness and Index

From the above figure we can see that power transfer between waveguides is very sensitive to refractive index and thickness of the waveguides. A single nanometer change in the waveguide thickness from the designed value would produce 10 micron change in coupling length and 30\% change in the coupled power transfer. This also corresponds to a change of 0.003 in the beta value. Since the device is supposed to measure sensitive 
changes the model is also very sensitive. Hence the depositions should be highly accurate to an extent of nanometer for efficient device operation. Only top waveguide based simulations were performed as the fabrication procedure adopted would provide the opportunity to compensate the bottom waveguide thickness or index variations.

Though this fabrication process gives a good opportunity to optimize the bottom two layers it also poses difficulties in depositing the third layer as it is altogether a new growth. This often results in thickness and index of top waveguide vary lot than required. Moreover the bottom layers have to undergo another deposition process which includes heating the layers at 300C for 3-4 hours. Though the films are stable they still might undergo change due to long thermal exposure and may result in intermix layers. Hence a new fabrication technique where all the three layers are grown at a time is developed. This would aid in producing the waveguides of the same indices and also gives a uniform thickness control. Since all the layers are grown at the same time a window for differentiating waveguides is done by etching top waveguide using shadow mask. Also the top waveguide can be etched back to tune it to resonant structure if necessary.

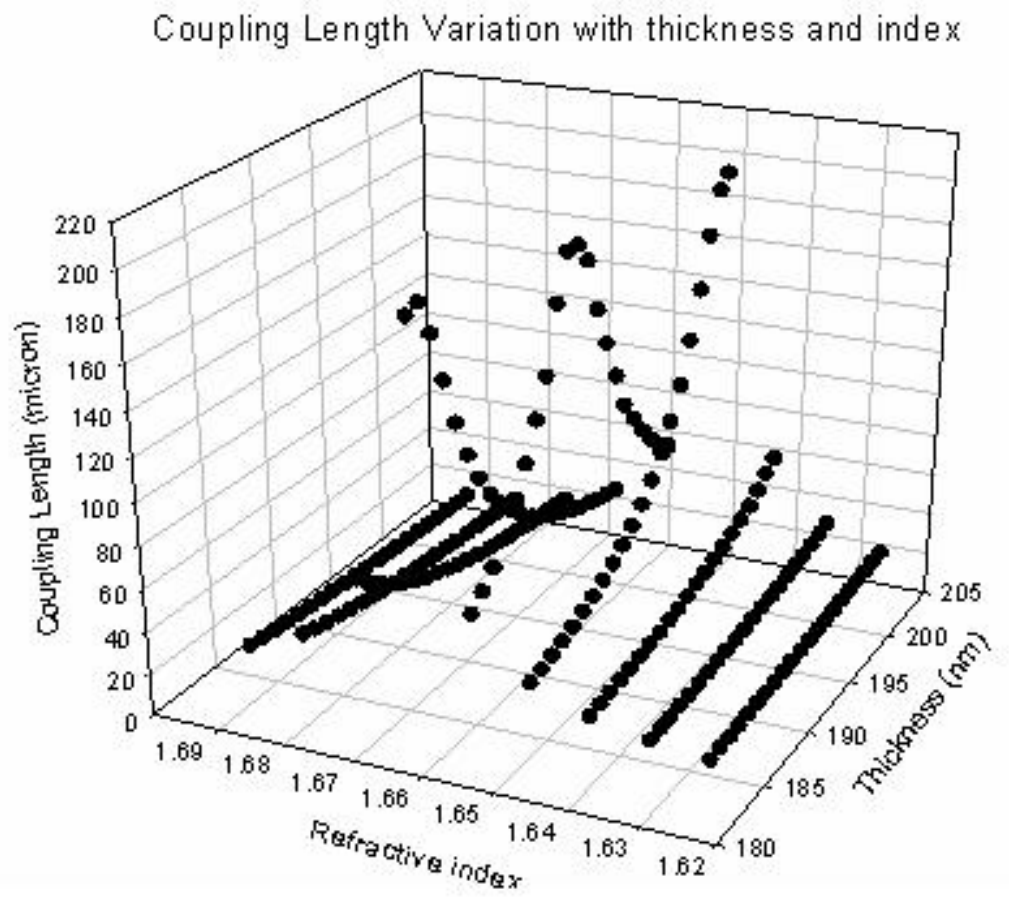

Fig 4.12(b) Coupling Length Variation with top waveguide thickness and index variation 
Since etching gives us a new etched surface, scattering loss and biolayer growth were the two issues to look at. It was proved that the scattering loss produced by the etched surface before and after the etch were in the measurement limits.. Fig 4.13 shows the guiding streaks before and after the etching. It can be observed that the streak is not greatly affected. AFM measurements may be insightful in this regard and were planned, however equipment issues resulted in measurements not being completed in time for publication of this work.

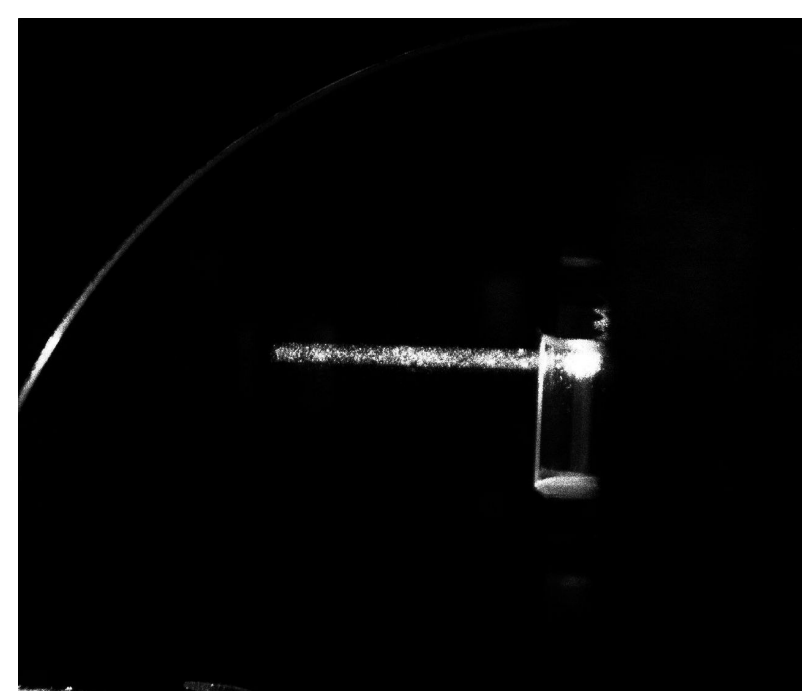

Fig4. 13(a) Before Etching

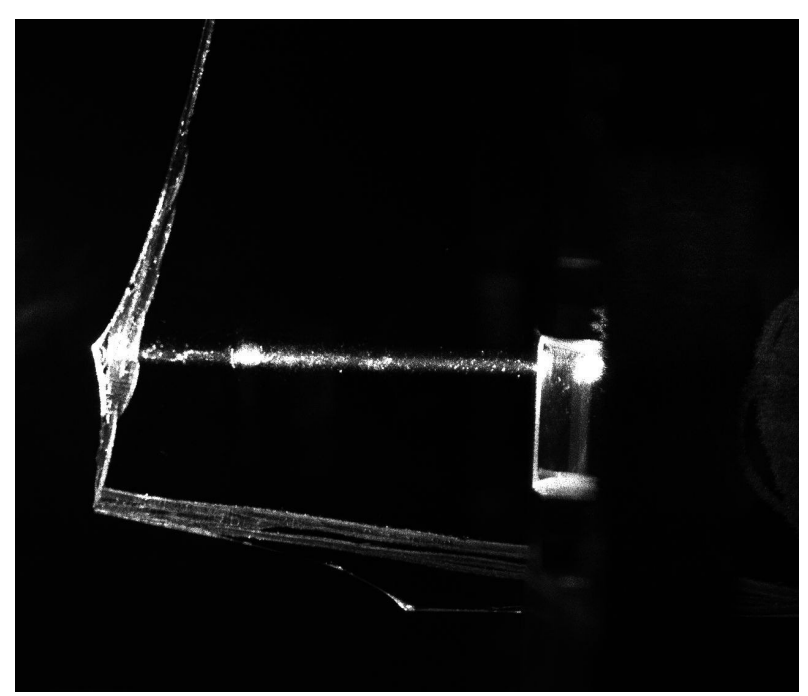

(b) After Etching

\subsubsection{Significance of sensitivity analysis:}

To understand sensitivity analysis we need to know the operation limits of the device. The first of them is the refractive index of the liquid flowed in the channel. The refractive index of the streptavidin chain is in the range of 1.4-1.5. The bio-layer is constituted of SAMs (self assembled monolayer), Streptavidin, Biotin and Fluorescent beads conjugate. We believe that the effective refractive index of the whole bio-layer would be in the range of 1.4-1.5 as all the above mentioned constituents have the refractive index in that range. Since the refractive index of water is 1.33 the effective refractive index of the whole bio-layer with water is expected to vary from 1.33-1.45 depending on the percentage composition of the bio-layer, water and also the percentage surface area covered by bio-layer. For flow cell experiments we chose glycerol solutions 
whose indices can vary from 1.3318-1.4716 [41] based on the mass fraction of glycerol and is near to the operation range of the device. Also the index of PDMS chip we use has an index ranging from 1.41-1.45. From the device operation point of view there should not be any power transfer at this index range. So effectively the fabricated device should be tuned in the range of 1.33-1.39 for device operation.

As an example let us consider a structure grown by Ion Assisted e-beam deposition technique which has the parameters as shown in the fig 4.3. Here ' $n$ ' is the refractive index of the film and ' $\mathrm{d}$ ' is the thickness corresponding to it.

\begin{tabular}{|c||}
\hline Alumina $n=1.6273 \quad d=253.136 n m$ \\
\hline Silica $n=1.458 \quad d=1234.033 n m$ \\
\hline Alumina $n=1.6273 \quad d=147.378 n m$ \\
\hline Borofloat $n=1.4773$ \\
\hline
\end{tabular}

Fig4. 14 Sample structure

For the structure shown in Fig 4.14 the electric field distribution when water is present on top waveguide is shown in Fig 4.15a. We observe that the total power is concentrated in the top waveguide. Since the device operation needs power transfer between the waveguides in the presence of water the top waveguide thickness has to be decreased. This can be achieved by etching the top waveguide. The thickness the top waveguide has to be etched can be found from the power transfer variation plot similar to Fig 4.12a. 
Dptical Field

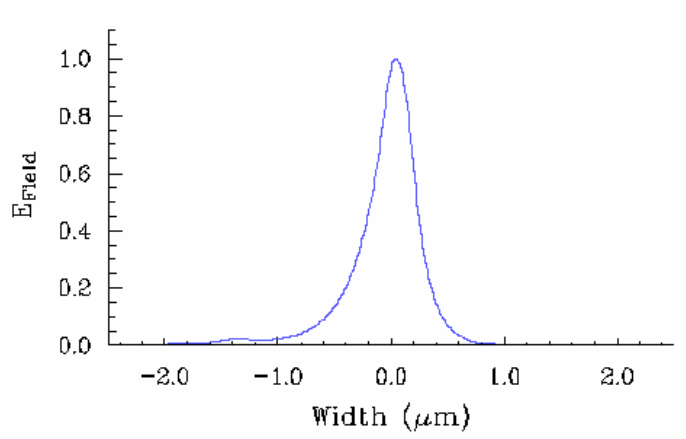

Fig4. 15 Top Waveguide Thickness (a) $d=253.136 \mathrm{~nm}(b) d=216.3 \mathrm{~nm}$

Also if we happen to etch the top waveguide more than needed, then tuning can be still be done by increasing the index of refraction of the liquid of the analyte. Power in the waveguides can be anticipated with the power variation graphs at different thickness of the waveguides. A two dimensional plot that shows the power transfer variation with top waveguide thickness for the above structure is shown in Fig 4.16. We see that there is maximum power transfer when the top waveguide thickness is $216.3 \mathrm{~nm}$. Thus Fig $4.15 \mathrm{~b}$ has been obtained by using the thickness from Fig 4.16. Thus the sensitivity analysis plot with top waveguide thickness variation can be used to estimate the power in the waveguides and also to evaluate the thickness the waveguide has to be etched to achieve maximum power transfer. Similarly an analysis with different concentrations of glycerol has to be done to observe the behavior of the device.

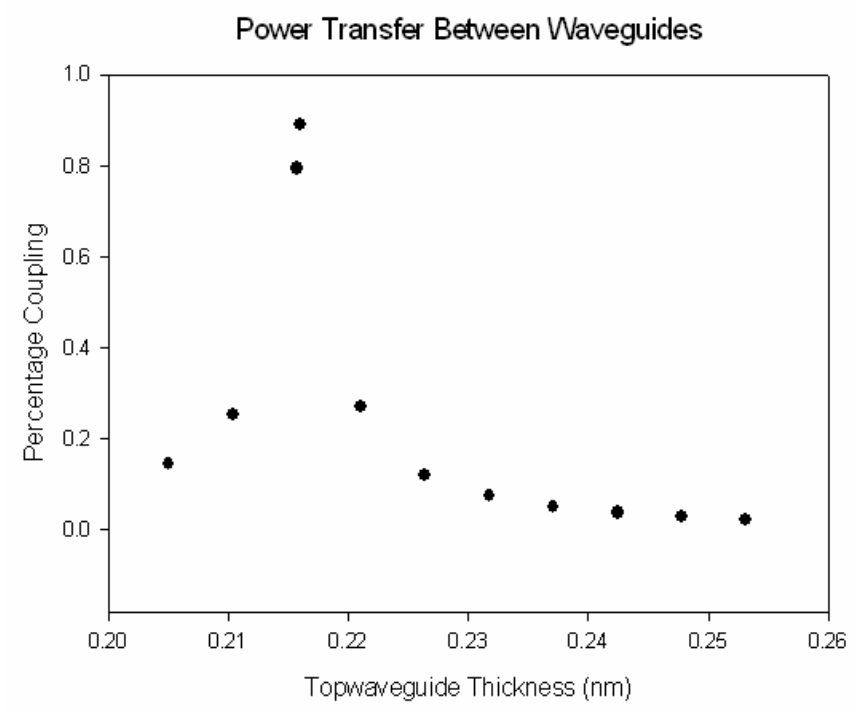

Fig4. 16 Power transfer variation between waveguides

\subsection{Flow Cell Experiments:}

Fig 4-17 shows the layout of the experimental setup used for performing the flow cell experiments. Fig 4.18a shows the PDMS micro fluidic channel with input and output 
capillary tubes setup used for flow cell experiments. Fig 4.18b shows the experimental setup used for performing the flow cell experiment. A PDMS mold is made using a silicon master made of SU8-25. The mold is bonded onto the top alumina waveguide using oxygen plasma exposure aided bonding method as explained in chapter 3. Inlet and outlet holes were drilled using a driller and capillary tubes were used for injecting liquid with an aid of a syringe pump. Syringe pump can inject fluids at very slow flow rates and thus pressure in the channel can be controlled. It can be seen in the layout that the top waveguide has been etched to differentiate the two waveguides. Quantum Imaging Retiga 1300 cooled digital CCD camera is used for power measurements. Harward Apparatus microprocessor based syringe pump has been used for pumping fluids into the channel.

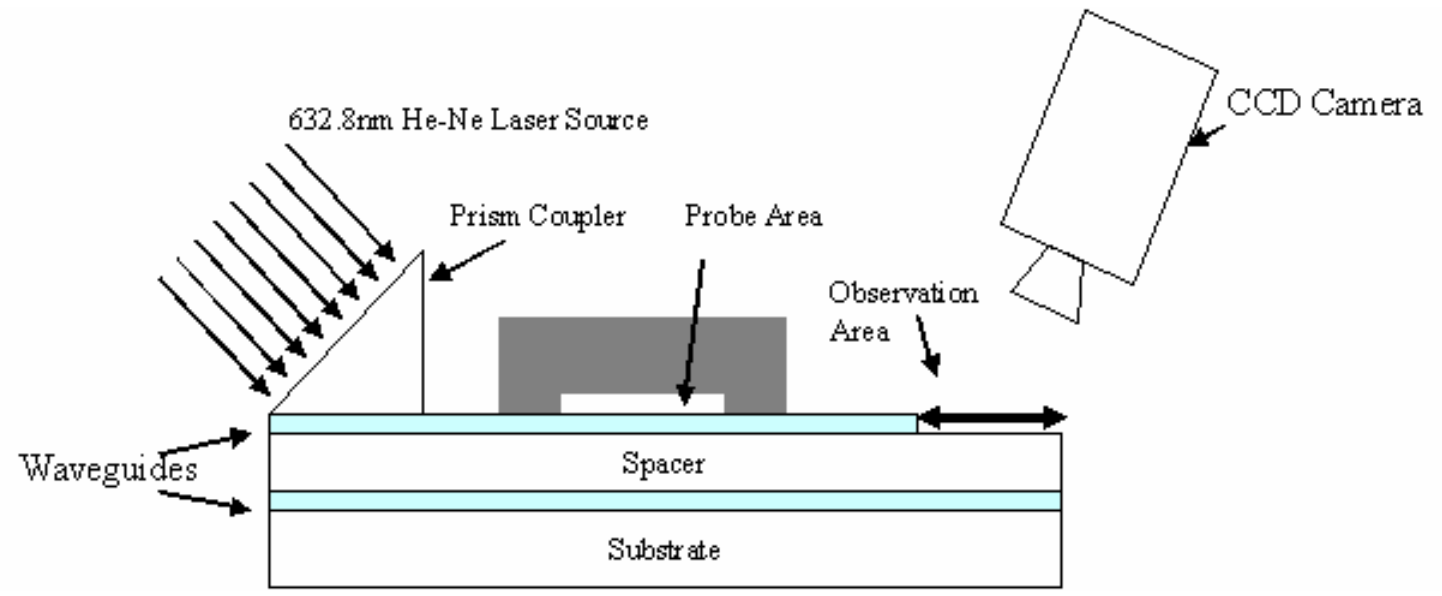

Fig4. 17 Layout of Experimental Setup

\subsubsection{Experimental Procedure:}

Light is coupled into the top waveguide with no analyte in the channels. An image with light coupled into bottom waveguide is also taken to compute the losses in the bottom waveguide alone. Analyte is pumped into the microfluidic channel with a syringe pump and images of the observation area are taken. Fig 4.17 shows the observation area to be imaged. The loss calculated in the observation area should be the same for all the cases and should be equal to the case when there is no analyte in the solution. 


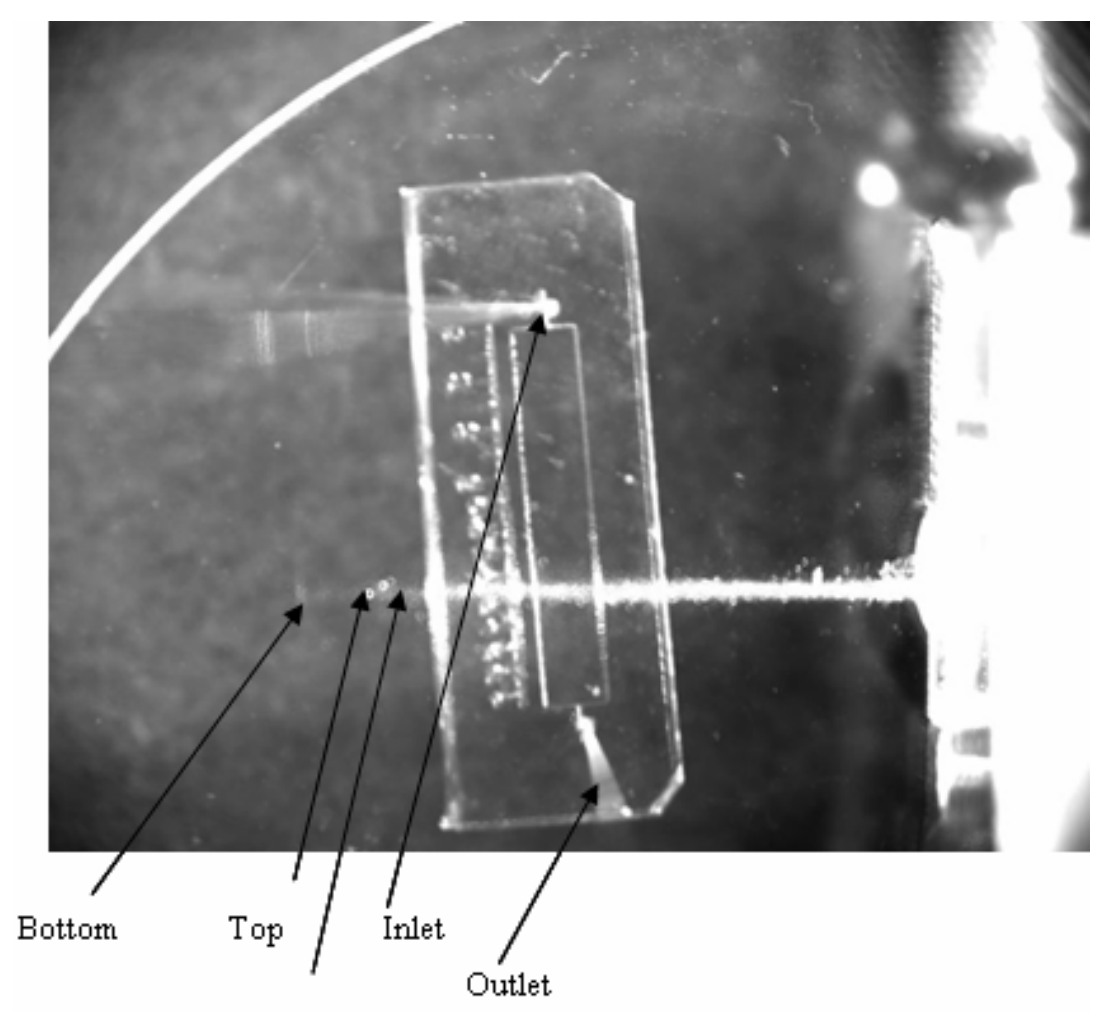

Streak in top waveguide

Fig4. 18a Film stack with PDMS channel

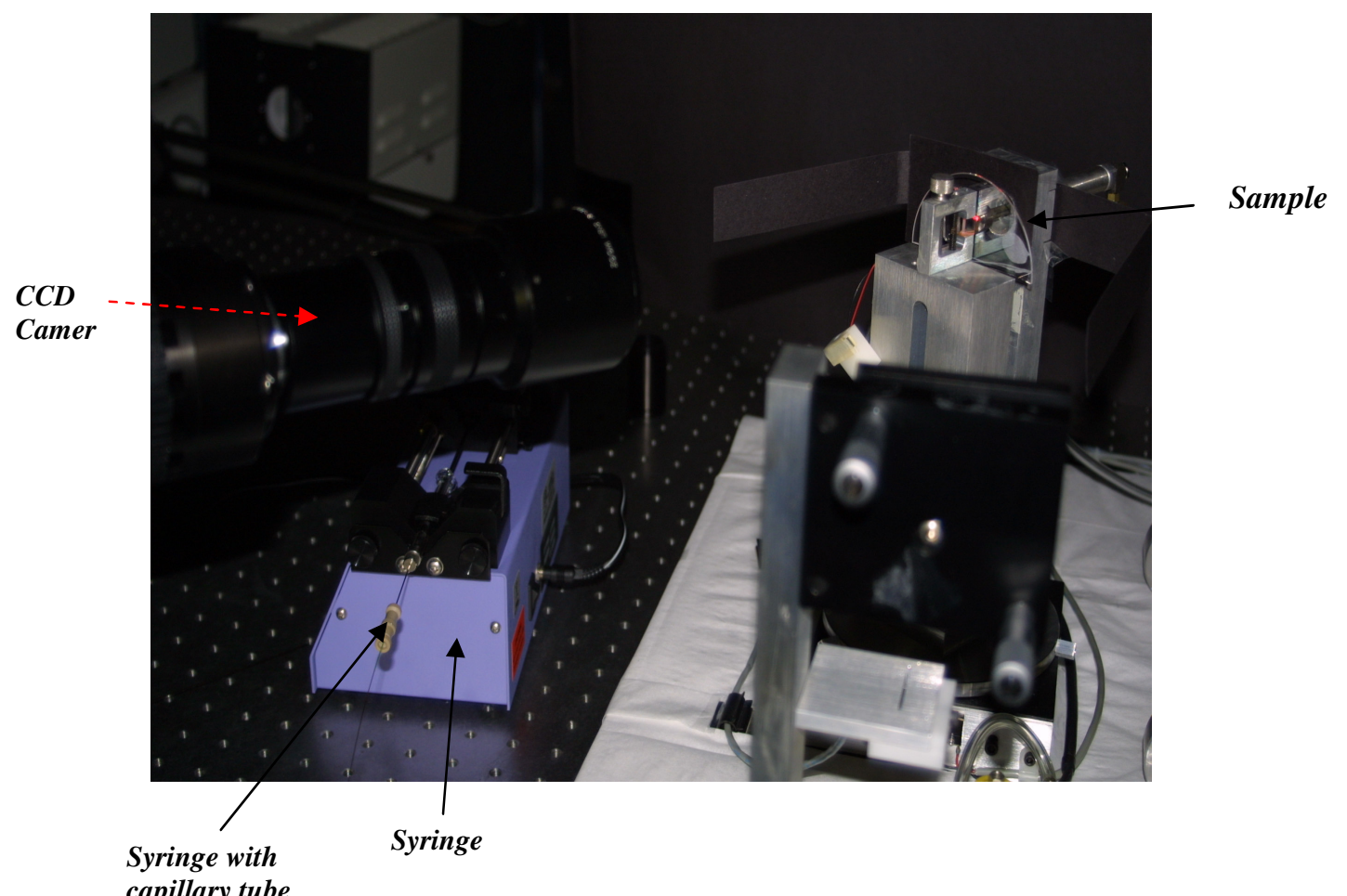

Fig 4.18b Experimental Setup for flow cell experiments 
Fig 4.19 shows the output obtained when water is injected through the channel. The laser light from He-Ne source is coupled into the top waveguide and the power is observed in the observation area. The power was not detected in the observation area as the light streak ended in the top waveguide after passing through the PDMS interfaces and channel interaction area. Based on the model from Ellipsometer the structure should support total power transfer in the channel area but this has not been observed. We speculated that the model employed in Ellipsometer to measure thickness and index is not appropriate. Details about this modeling would be discussed in later part of the thesis.

Though power was not observed in the observation area, analysis for the detection of bio-agents is as follows. In Fig 4.16, the red colored portion has been taken for analysis. As can be seen it is the whole observation area with the streak.

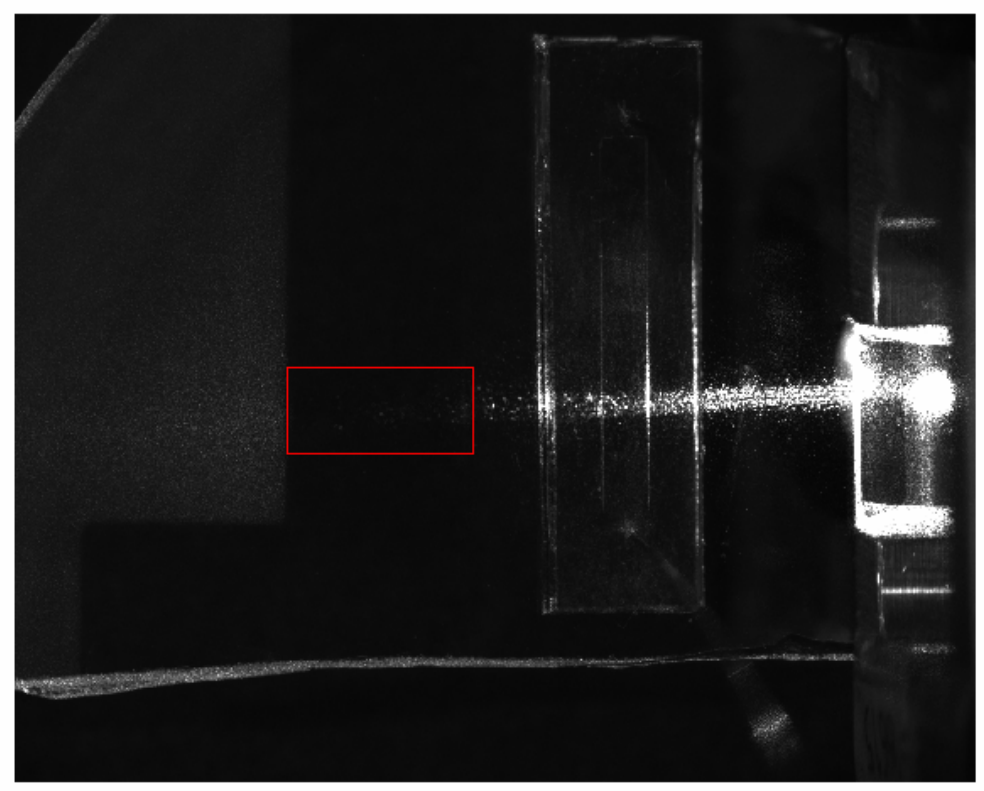

Fig4. 19 Image obtained with water in the channel

The procedure for detecting the analyte refractive index is as follows. An image before any analyte pumped is captured and loss is computed in the observation area using the procedure mentioned in Appendix B. Analyte would be pumped into the channel and the image is to be taken. It is necessary to focus on the observation area and get the maximum information as the detection is based on the intensity of light detected at the 
ends of the waveguide. Ideally the presence of analyte would reduce amount of power transfer between the waveguides and the light intensity observed in the observation area changes depending on the interaction length and coupling length of the model. So depending on the index of the analyte present the intensity decreases with the maximum intensity present when the analyte is water (assuming that the structure is designed to tune for water). Losses for each case are computed using the procedure mentioned in Appendix B. Ideally the losses be the same in all the cases but at different intensity levels. In other words, loss in the waveguide is constant irrespective of the index of the analyte present in the channel. The presence of analyte effects only on the intensity of power seen in the observation area. Fig 4.20 shows the graph that should ideally be seen for different analyte solutions.

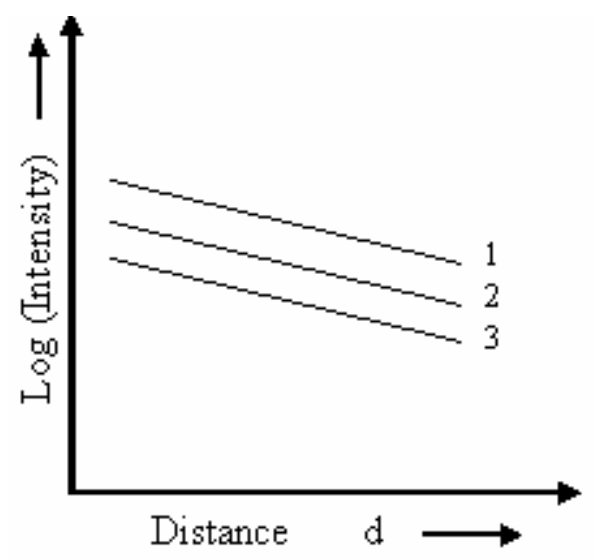

Fig4. 20 Loss variation in the observation area for different analytes

\subsubsection{Detection of the Analyte:}

Images before and after analyte flow are taken using a CCD camera. The sum of pixel intensities along columns is computed for both images. The relative changes in the column intensities with and without analyte were compared with the simulation results to detect the analyte [18]. The relative change in the intensity is prone to coupling changes at the prism. Hence extreme care is necessary to avoid any change in the physical alignment of the sample during analyte flow. Any change in observation area would change the measurements. Also the average column pixel intensity would depend on the rectangular box selected in the observation area. If the selected observation area is large, the average pixel intensity would be much prone to noise. 
Fig $4.21 \mathrm{a} \& \mathrm{~b}$ shows the power variation in the waveguides when the index of the Biolayer is 1.3318 and 1.34 respectively. The relative change in the power from simulations is used to compare with the experimental values. Let $\mathrm{P}_{1}$ and $\mathrm{P} 2$ be the powers observed in the observation area for refractive indices $\mathrm{n} 1$ and $\mathrm{n} 2$ respectively. The relative change in intensity is given by $(\mathrm{P} 1-\mathrm{P} 2) / \mathrm{P} 1$ and can be compared with the experimental values. Powers obtained from experiments must correspond to the same physical area.
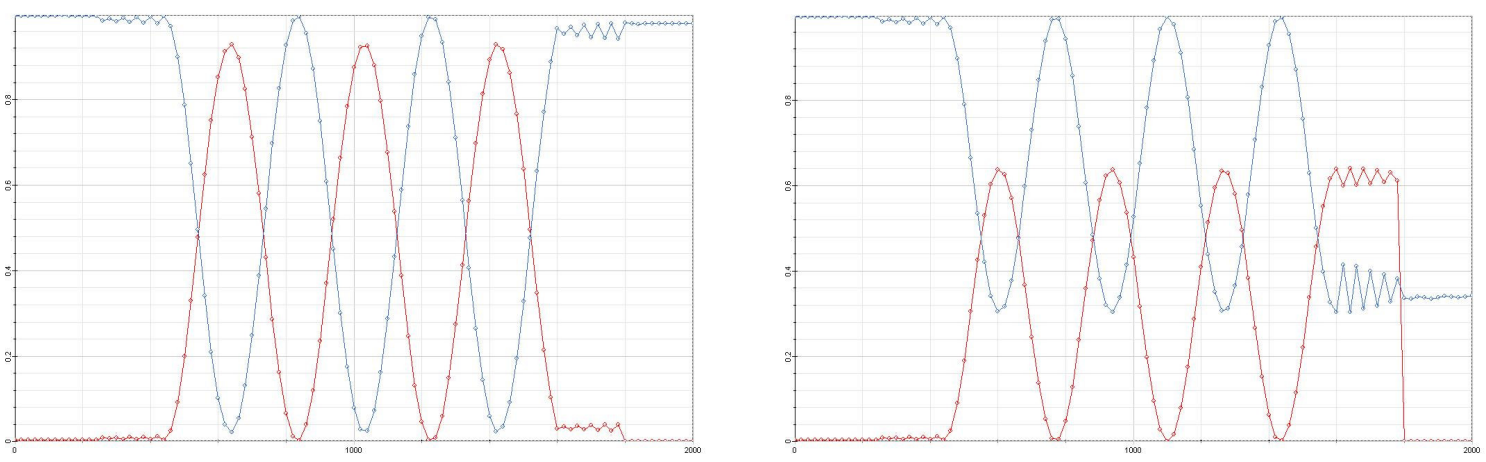

Fig4. 21Globally normalized power in waveguides (a) $n=1.3318$, (b) $n=1.34$

Fig 4.22 shows the image taken before any water flow in the channel. The observed area is marked white on the image. Another image is taken without disturbing the alignment of the sample but with water flowing in the channel. Fig $4.23 \mathrm{a} \& \mathrm{~b}$ shows the observation areas for before and after water flow cases. We observe that there is change in the pixel intensities of the region. Also there is significant noise present in the region which can influence the measurements. Hence efficient techniques have to be developed to overcome the effect of spurious scattering points. 


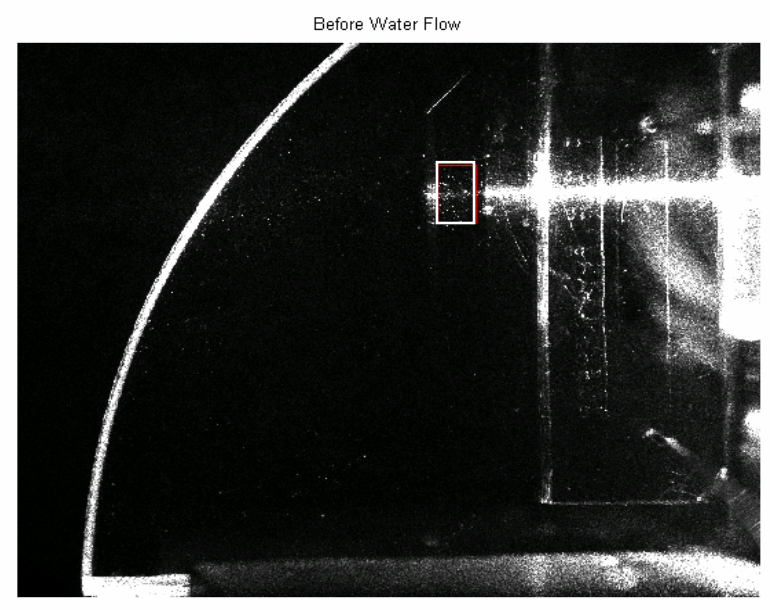

Fig4. 22 Before water flow

Selection of regions with less scattering points is one such solution. The selection of the area for analysis is also critical when calculating losses in the observation area. The larger the rectangular box the larger is the effect of noise on the measurements, unless the appropriate normalization is chosen. Slopes of the column intensities are calculated for both the cases and the slope of the logarithm of pixel intensity is computed.

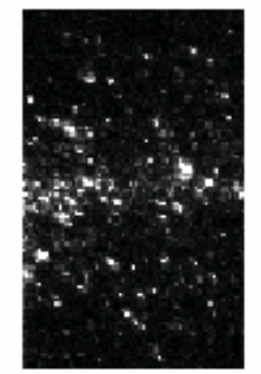

Fig4. 23Observation area a) Before water flow

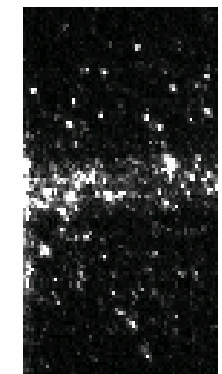

b) After water flow

Fig 4.24 shows the slopes of the two images. While the intensity varies, both the slopes should be the same as discussed in previous section. 


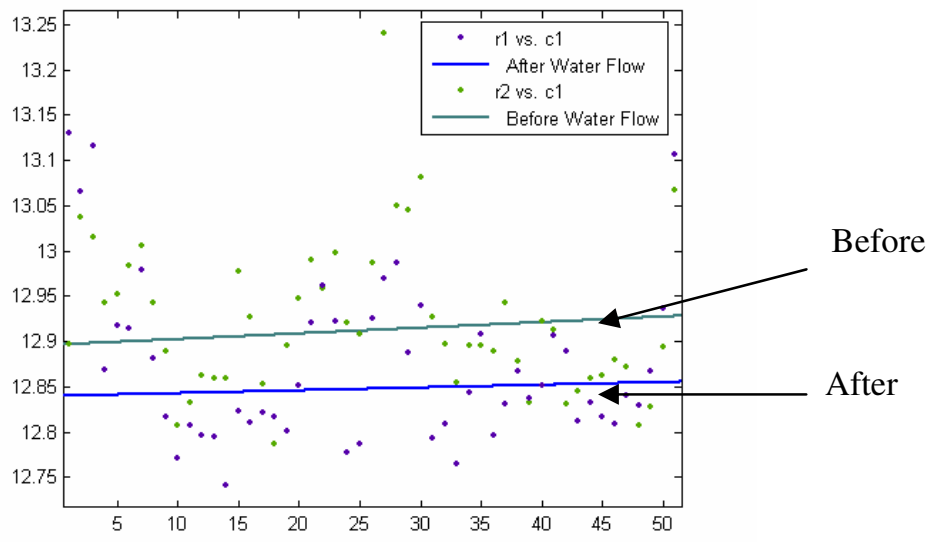

Fig 4.24 Slope Before: $0.0004+/-0.0008$ After: 0.0006

\subsection{Issues with Ellipsometer Measurement/Modeling:}

Fig 4.19 shows a device structure that did not show power transfer while simulations based on ellipsometer measurement and its internal film models showed complete power transfer. Hence in order to validate the accuracy of the ellipsometer, the following process was adopted. A portion of the sample was etched till the bottom waveguide was reached in order to measure it separately given that a very low index for the film was indicated by the ellipsometer measurement. The bottom waveguide index after etching was found to be graded with maximum index seen at the substrate as shown in Fig 4.25. Graded index have not been found in the single layer films grown. The thermal and environmental cycling arising during second and third layer depositions may be the cause of this behavior; however the validity of the ellipsometer measurement must be established as well. In some cases two different models with low mean square error are obtained from ellipsometry and neither of them matches with the experimental result from the prism coupler. These issues are being investigated. 


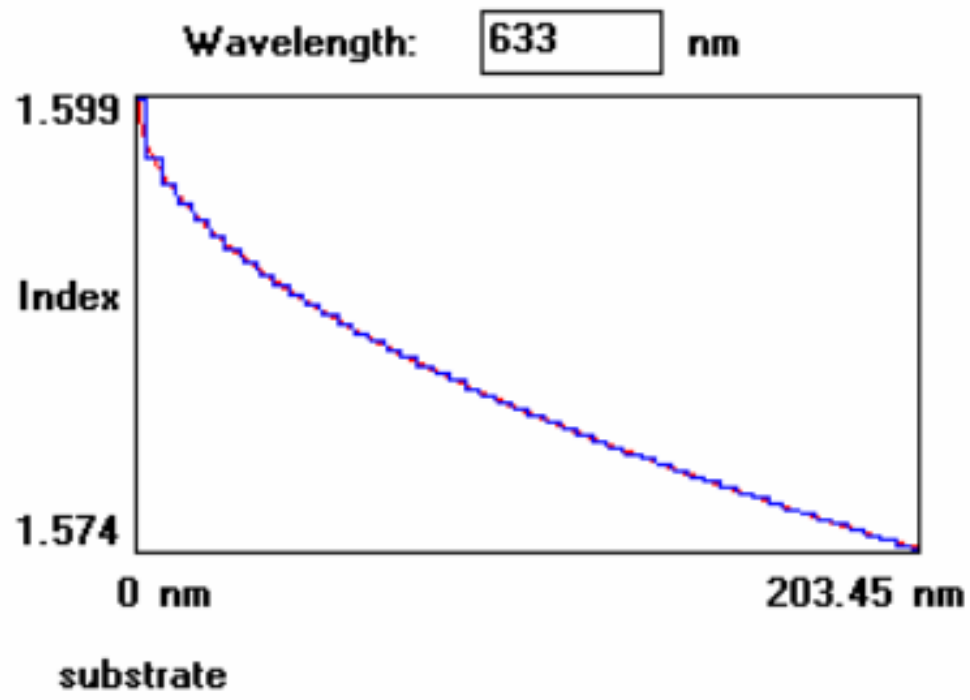

Fig4. 25 Index variation of the bottom waveguide after etching 


\section{CHAPTER 5: Conclusions and Future Work}

The simulations and experimental work to date have shown that the designed architecture has the potential to serve as a transducer for bio-sensing. Sensitivity analysis has been performed to check the limitations of the device. It was found that the device operation is highly dependent on the thickness and index accuracy of the waveguides. A single nanometer change in thickness would result in $10 \mathrm{~nm}$ change in the coupling length and $30 \%$ change in the coupled power, hence the device has to be fabricated strictly to tolerance. A PDMS based micro fluidic channel has been successfully hybidly integrated with the SPARROW film stack. This produces significantly less strain on the sample and doesn't change the prism coupling spot conditions when under test. A process for bonding PDMS based micro fluidic channel on alumina waveguide was developed. A system was developed including adoption of a syringe pump in order to pump different concentrations of analyte into the channel at controlled flow rates. Further ongoing advances in stack growth should enable effective flow cell experiments.

A process for optical characterization of alumina waveguides has been developed to calculate the losses of the waveguide. Matlab code has been written to evaluate the losses and filters have been used to minimize the scattering effects. Waveguides with high losses and more scattering represent a continuing challenge for loss evaluation. Exploration of filtering and data analysis techniques can be further employed to overcome this problem.

Optical loss variation of alumina waveguides as a function of ion beam parameters like drive current and oxygen flow rate has been explored. For the current deposition technique, losses increase with ion drive current and decrease with oxygen flow rate. The reasons for this behavior though are yet to be known.

Sensitivity analysis has been performed with respect to fabrication of the top waveguide but can be extended to the bottom waveguide also. An important issue to be addressed is the ellipsometer measurement and modeling of multi layer films. An accurate model or protocol has to be developed to characterize the waveguide stack. 
The next step in the work is for flow cell experiments to be performed for different index fluids and analytes and the responses are to be verified with simulation results. Ongoing efforts should enable the evaluation of the designed structure to be completed and its efficacy as a transducer fully determined. 


\section{Bibliography:}

[1] Biosensors and other medical and environment probes http://www.ornl.gov/ info/ornlreview/rev29_3/text/biosens.htm.

[2] http://www.nanovic.com.au/?a=investments.biosensor\&p=216.

[3] Clark, L.C. and Lyons, C., (1962) Electrode systems for continuous monitoring in cardiovascular surgery, Ann.NY Acad. Sci., 102.

[4] Vastarella W., Enzyme Modified Electrodes in Amperometric Biosensors

[5] D.Lloyd, L.Hornak, S.Pathak, D.Morton, and I.Stevenson, (2004) "Application of Ion Beam Assisted Thin Film Deposition Techniques to the fabrication of Biosensor Chip With fieldability Potential for Important Biohazard Detection Applications" 47Annual Technical Conference Proceedings, Soc of Vac. Coaters, ISSN 0737-5921

[6] Mark S. Vreeke, Amperometric Detection, http://www.devicelink.com /ivdt/archive/97/07/010.html

[7] Tescione, L., An Introductory Tutorial of Biosensor Technology, http://www.rpi.edu /dept/chem-eng/Biotech-Environ/BIOSEN/basicsensor.html

[8] Basic theory of ISE measurements, Retrieved Oct 18, 2006, http://www.nico2000.net /Book/Guide4.html

[9] Martin Chaplin, Christopher Bucke, Enzyme Technology, http://www.lsbu.ac.uk/ biology/enztech/biosensors.html

[10] http://www.crh.org/advanced_technology/AdvancedTechnologyFall2005.pdf

[11] Poultry tech, Retrieved Oct 8, 2006 from http://atrp.gatech.edu/pt17-3/17-3_p2.html

[12] Wang J., (2005 Dec 1), Electrochemical biosensors: Towards point-of-care cancer diagnostics, Biosens Bioelectron

[13] Hollars CW, Puls J, Bakajin O, Olsan B, Talley CE, Lane SM, Huser T., (2006, June 27), Bio-assay based on single molecule fluorescence detection in microfluidic channels, Anal Bioanal Chem.

[14] Bard AJ, and Faulkner LR, Electrochemical Methods, New York, John Wiley \& Sons, 1980

[15] Ashok Kumar, Biosensors based on Piezoelectric Crystal Detectors: Theory and Application http://www.tms.org/pubs/journals/JOM/0010/Kumar/Kumar-0010.html 
[16] Naveen Paturi, Chinnayya R. Vemuri, Dimitris Korakakis, Lawrence A. Hornak., (2005, Dec), "Analysis of cluster defects in Photonic Crystals for biosensor applications", Proceedings of SPIE Vol. 6005, pp 146.

[17] Evanescent Waves, Carnegie Mellon Univeristy http://www.andrew.cmu.edu/user/ dcprieve/Evanescent\%20waves.htm

[18] Pathak, S., (2004), "Resonant Optical Waveguide Biosensor Characterization", Masters Thesis, West Virginia University.

[19] S.J.Updike, G.P.Hicks, Nature, 214 (1967) 956; Science, 158 (167) 270

[20] Donald L. Lee, “Electromagnetic Principles of Integrated Optics”, John Wiley \& Sons

[21] A. Bruce Buckman, "Guided Wave Photonics”, Sauders College Publications.

[22] R.G. Hunsperger, "Integrated Optics: Theory and Technology", $2^{\text {nd }}$ Edition, Springer-Verlag publications.

[23] R.P. Khare, “Fiber Optics and Optoelectronics”, Oxford University Press

[24].Wang Zhi, Ren Guobin, Lou Shuqin, and Jian Shuisheng, (2003) "Loss properties due to Rayleigh scattering in different types of fiber" Optical Society of America. Vol: 11.

[25] Joseph Palais, "Fiber Optic Communications" $5^{\text {th }}$ Edition, Prentice Hall Publications.

[26] Liang An-Hui, 'The Rayleigh Scattering Loss of Isotropic Weakly Guiding SingleMode Waveguides', IEEE Journal of Quantum electronics

[27] R.Ulrich and R.Torge, (1973), Measurement of Thin Film Parameters with a Prism Coupler, Applied Optics 12, 2901-2908.

[28] Documentation for Prism Coupler, Metricon Corporation, Pennington, NJ.

[29] Optiwave Documention for OptiBPM 7.0, Optiwave Corporation, Ottawa, ON, Canada

[30] Wei-Ping Huang, (1994, Mar 11), Coupled-mode theory for optical waveguides: an overview, J.Opt.Soc.Am.A, Vol 11.

[31] Weiping Huang, Sujeet K.Choudhary, (1993 March), "Variational Coupled-Mode Theory of Optical Couplers", J. light wave technology, Vol 8.

[32] Nano SU8 25 Specifications Sheet. 
[33] Gregory T.Roman, Tyler Hlaus, Kevis J. Bass, Todd G. Seelhammer, and Christopher T. Culbertson, (2005 March), "Sol-Gel Modified Poly(dimethylsiloxane) Microfluidic Devices with High Electroosmotic Mobilities and Hydrophilic Channel Wall Characteristics”, “Analytical Chemistry”, Vol 77, No 5.

[34] Wright J.D., (2001), "Sol-gel materials: chemistry and applications", Gordon and Breach Science Publishers.

[35] Shantanu Bhattacharya, Aridom Datta, Jordan M Berg and Shubhra Gangopadhyay, (2005, June), "Studies on Surface Wettability of Poly (Dimethyl) Siloxane (PDMS) and Glass Under Oxygen-Plasma Treatment and Correlation With Bond Strength," J.Microelectromechanical Systems, Vol. 14, No 3.

[36] Cornell T., (2005), "Fabrication of Resonant Optical Waveguide Biosensors using Electron Gun Depositions", Masters Thesis, West Viriginia University.

[37] Vikas A, Sheela A., "Measurement of loss and mode-profile for integrated optic waveguides using a camera", Proceeding of SPIE, Vol 4579

[38] Thomas A Strasser, Mool C.Gupta, (1992, April), "Optical Loss Measurement of low loss thin-film waveguides by photographic analysis", Applied Optics, Vol 31, No 12

[39] Ellipsometer Documentation, J.A.Woollam Company Inc., Lincoln, Nebraska.

[40] Praneetha P., (2007), "Fabrication and functional analysis of SPARROW biosensor", Masters Thesis, West Virignia University.

[41] Kim B.J., Liu Y.Z., Sung H.J., (2004), "Micro PIV measurement of two-fluid flow with different refractive indices", Meas. Sci. Technology. 15, 1097-1103. 


\section{APPENDIX A \\ OPTIBPM 7.0 Simulations and Modeling Parameters}

\section{Guidelines for Execution of Simulations}

- Use Modesolver 2D for calculating effective refractive indices and thereby beta values. OPTIBPM 7.0 has some assumptions and would not give correct modal indices. Compare them with the ones seen in the prism coupler.

- The input waveform for the waveguide stack if done from the BPM tool would not give the correct modal indices as it assumes the wafer on either side of the waveguide. The best method is to get the input wave using $2 \mathrm{~d}$ Mode solver. Remember to have same mesh size and the wafer size when you do this.

- Scripting can be done to vary the thicknesses of the waveguides or wavelength of operation but we have to incorporate the shifting of the waveguide offset due to the change in the thicknesses. (Not necessary if parameter is wavelength).

- Path Monitor and slice selector options can prove very helpful to observe the field in the waveguides.

- Use of parameters and varying them as a series could prove helpful in determining the behavior of the device when the thickness and indexes are off.

\section{Input types in OPTIBPM 8:}

There are many ways of giving input to the designed structure. The general input files that are found in the software are Modal, Gaussian, Rectangular and through File. Depending upon the excitation given in reality a corresponding input wave is selected. Since the biosensor we use is excited using a Prism Coupler at certain modes we might be inclined to use modal input for performing the simulations. There are certain assumptions in using Modal input which are dealt in the next paragraph.

In modal input excitation a waveguide is selected from the design and the mode is calculated assuming that the clad and substrate indices are of the wafer. In all practical uses even a single layer waveguide based device would also be an asymmetric waveguide and the input if used through this way will always be calculated for a symmetric waveguide. This would work fine for single waveguide designs and for lesser accurate 
designs. But for multi layer films which have two or more waveguides it is always recommended to design the whole structure in " $2 \mathrm{~d}$ Mode Solver" and calculate the modes. Once a modal structure is obtained it can be saved as an .f2d file. The same can be used as an input to the structure used in the simulation. But the number of mesh points and the wafer width has to be the same in " $2 \mathrm{~d}$ Mode Solver" and OptiBPM 8 to perform the simulations. Since we are using input file separately, file checkbox has to selected for this type of calculations.

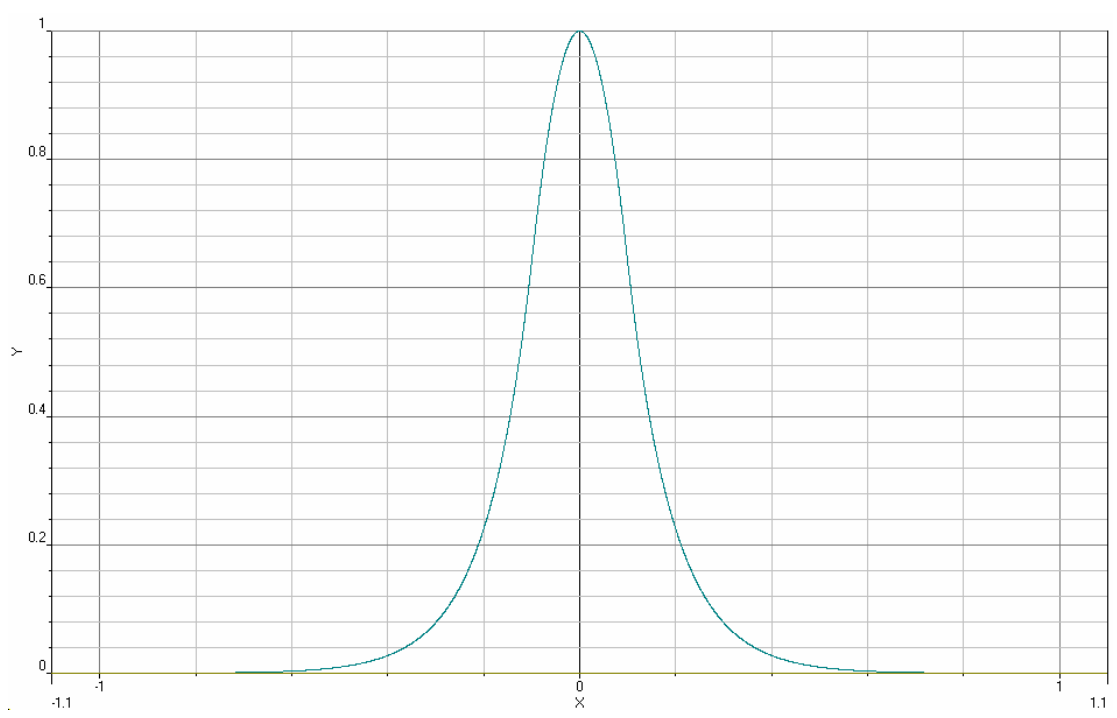

Fig 1 Mode Profile of a single layer waveguide

\section{VB Scripting for Parametric Analysis:}

It is important to understand the variation of the output quantities with variation in a designed parameter. The usual parameters that can change are the input wavelength, index and thickness of waveguide. A simulation can incorporate a change in one of the following quantities but it is to be noted that the input wave remains the same for all the variations. This variation can be incorporated in simulations using scripting. A sample script for wavelength variation is written below.

Const Numiterations $=3$

lambda $=1.55$

For $\mathrm{x}=1$ to Numiterations

ParamMGR.SetParam "wavelength",CStr(lambda)

ParamMGR.Simulate 
WGMgr.Sleep(50)

lambda=lambda- 0.15

Next

It is difficult to vary the thickness of the waveguide as thickness variation would bring a change in the physical location and all the other layers in its vicinity are to be shifted accordingly to compensate the thickness change since no gap can be allowed between the waveguide and its adjacent layer. Hence offset compensation has to be incorporated in all the layers of the design when performing this kind of simulations. The other problem present with this model is the input waveform. It remains constant for all the iterations and does not incorporate the thickness change. Though this does not produce appreciable error for little variations it is still inaccurate. Hence the user has to understand the following problems and errors and use this method judiciously.

\section{Path Monitor and Power at the ends of Waveguide:}

'Path monitor' can be used to see the variation of power in the designed waveguides and 'Power in Output Waveguide' can be selected to observe the powers at the ends of the waveguide. This can prove helpful if the variations are less and which cannot be observed visually.

\section{Process for computing a simulation for structure:}

Fig 2 is the structure for which the TE simulation would be performed. Light would be input in the bottom waveguide and optical field intensity would be observed. The same structure is designed in " $2 \mathrm{D}$ Mode solver" to obtain the input wave. But for the input wave the top layer is assumed to be air since during prism coupling the modal input would be due to air.

Fig 3 shows the input wave obtained using mode solver with the top layer index of air. Since in BPM simulation tool the input can be given only at the center of the wafer the final thicknesses for water and Borofloat are to be found. The mesh was assumed to be 8000 . Assuming the thickness of water to be 1 micron the final thickness of Borofloat would be 2.294 microns $(1+0.194+1.1+0.144 / 2)$. And the whole thickness of the wafer is 
4.732 micron. These values are used in the BPM simulator with the same number of mesh points.

\begin{tabular}{|ll|}
\hline Water & $\mathrm{N}=\mathbf{1 . 3 3 1 8}$ \\
\hline Alumina & $\mathrm{N}=1.659 \quad \mathrm{~d}=194 \mathrm{~nm}$ \\
\hline Silica & $\mathrm{N}=1.457 \quad \mathrm{~d}=1100 \mathrm{rm}$ \\
\hline Alumina & $\mathrm{N}=1.659 \quad \mathrm{~d}=144 \mathrm{~nm}$ \\
\hline Borofloat & $\mathrm{N}=1.4701$ \\
\hline
\end{tabular}

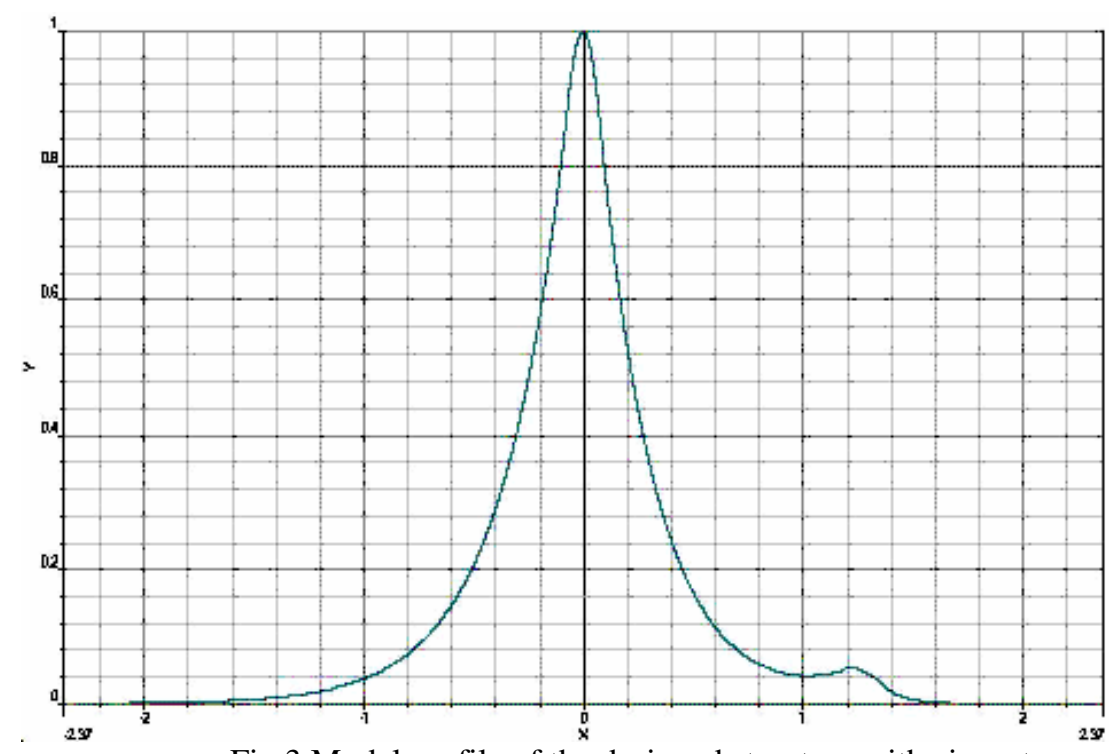

Fig 3 Modal profile of the designed structure with air on top

In the profiles and materials dialog box define the materials that are going to be used in the design under dielectrics tab. In the channels tab select each material that is going to be used in the design. In $3 \mathrm{~d}$ simulations these channels can be used to define certain shapes. Since we are concerned with only $2 d$ simulations we ignore the $3 d$ parameters. A screen shot of defined materials and channels is shown in Fig 4. 


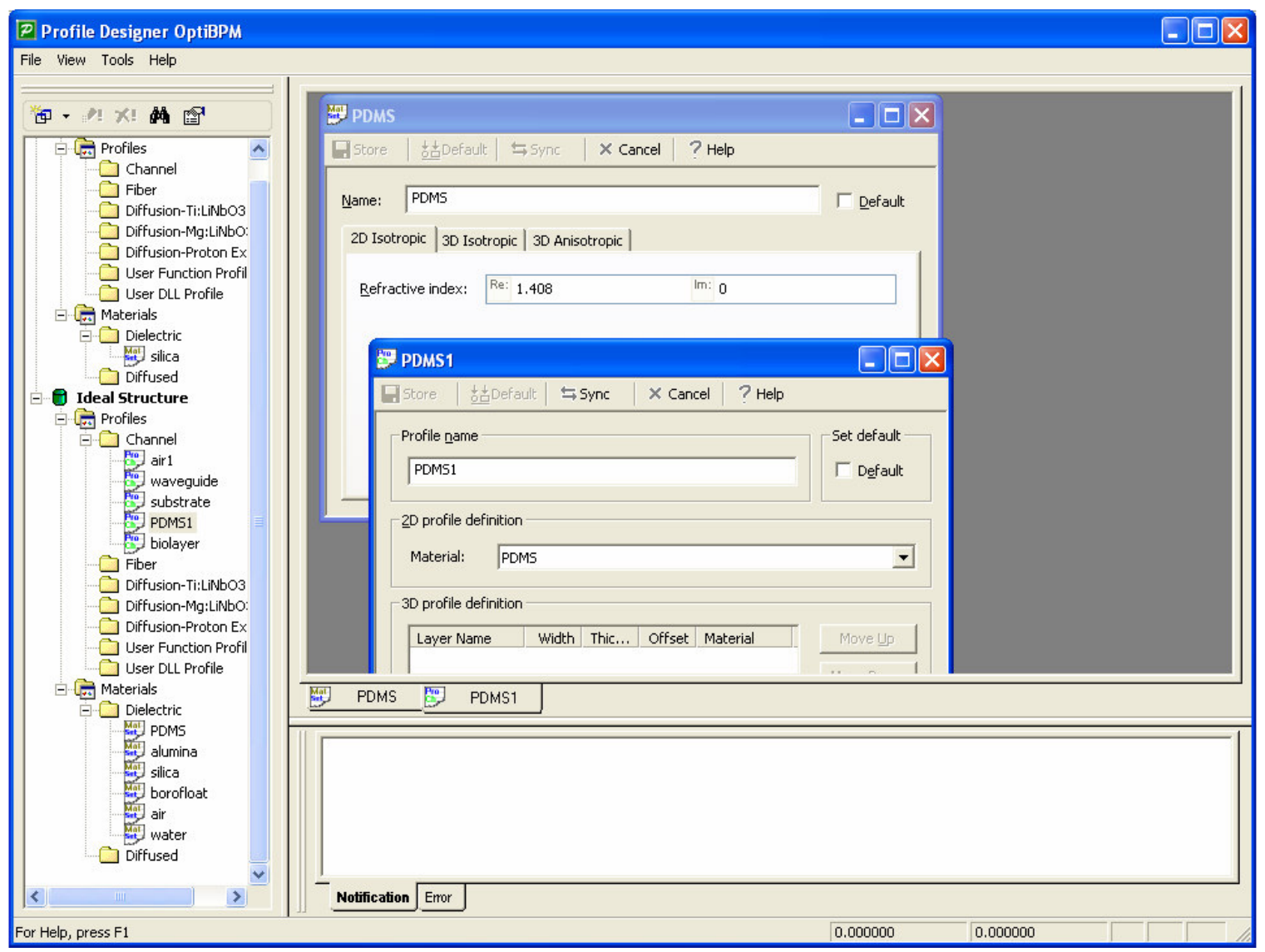

Fig 4 Profile Designer Window

Once the desired materials are defined the wafer properties dialog box can be filled. Select the wafer material from the designed channels and the width of wafer would be the one calculated from mode solver. The length of the wafer depends on the interaction length we choose to perform the simulation for. Default waveguide generally can be chosen any of the material as it can be changed in the design layout window. Since we are not concerned with the $3 \mathrm{~d}$ simulation we need to Once the wafer properties dialog box is filled hitting "OK" tab would takes us to the layout window. We can design the linear waveguides from "Draw" tab and the length and width of the waveguides are to be specified as per the design. It is to be noted that the waveguide is laid symmetric such that the location specified would be the axis of the waveguide. From the "simulation" menu "simulation parameter" is selected. Mesh points and wavelength are selected as per the mode solver.

Input plane is selected from draw menu and the "file" option is selected for giving input from another file. File obtained from mode solver is selected. Once the input is 
selected the next step is to perform the simulation. Extra features like path monitor, output power in waveguides can be selected depending upon the parameter of interest. 


\section{APPENDIX B \\ Loss Measurements using Matlab Program and Prism coupler:}

We have developed a method for computing the loss of the waveguides using prism coupler and Matlab code. A guiding streak is imaged with a CCD camera and is processed in Matlab to compute the loss of the waveguide. The process for the same is discussed here.

- Mount the sample onto the Prismcoupler and reference it if concerned with the beta values of the film.

- Once the effective indexes are calculated remove the detector and place the CCD camera focusing on the regions you like to measure.

- Place a graph paper on the back side of the sample and take measurement with light switched on so that it can be used as a reference for pixel to distance conversion.

- Without changing the focus of the camera take the images for different exposure times.

\section{Matlab Code:}

Copy the file latest.m and paste it in the folder containing the images. Open the file from Matlab and input the name of the image you want to measure the losses of.

There is a code which computes the average of the pixels around each point. Use this as necessary.

- The first step is to align the image as straight as possible since the image taken with graph paper may not be aligned properly. Code has already been written to align the graph but the user has to identify two points that he wants to be in a straight line.

- The next step is to compute the distance between the two points selected. Hence it would be easier if we select the two points between whose distance is already 
known. We select any two points on the graph paper and the distance between them can be obtained in terms of pixels.

- This value (pixel to distance ratio) would remain constant as long as the focus and the position of the camera does not change.

- The next step is to select the image whose loss is to be determined.

- A dark image should also be taken at the same time when the original images were taken to include the effect of dark current of the CCD camera.

- The dark image is subtracted from the original image which takes into account the effect of background.

- The new image has to be used for computing the losses.

- A code has been written to select a block of image from the given image. Hence the user can select the intended portion of analysis by using the mouse.

- Once a portion of the image is selected, it undergoes through a series of filters. The first filter is the scattering removal filter. A code has been written to identify the scattered pixels based on the intensity of the pixels. If the intensity of a pixel is very much larger than the average of the surrounding pixels then it is considered to be a scattered pixel and it replaced by an average value of the surrounding.

- Once an image is selected and filtering is done (latter is user's choice) the column intensities along each row are computed and are stored in a $1 \mathrm{~d}$ matrix.

- Logarithm of the matrix is taken and 'cftool' (Curve fitting Tool) is employed in Matlab to fit the selected points. It gives the slope along with $95 \%$ confidence levels too. 


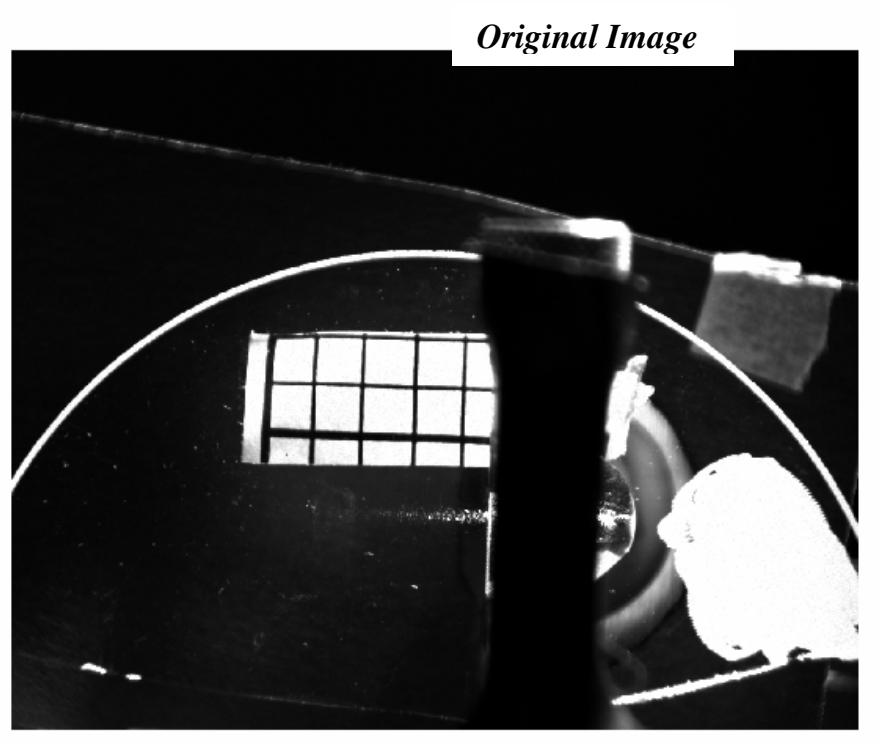

After Rotation of the image

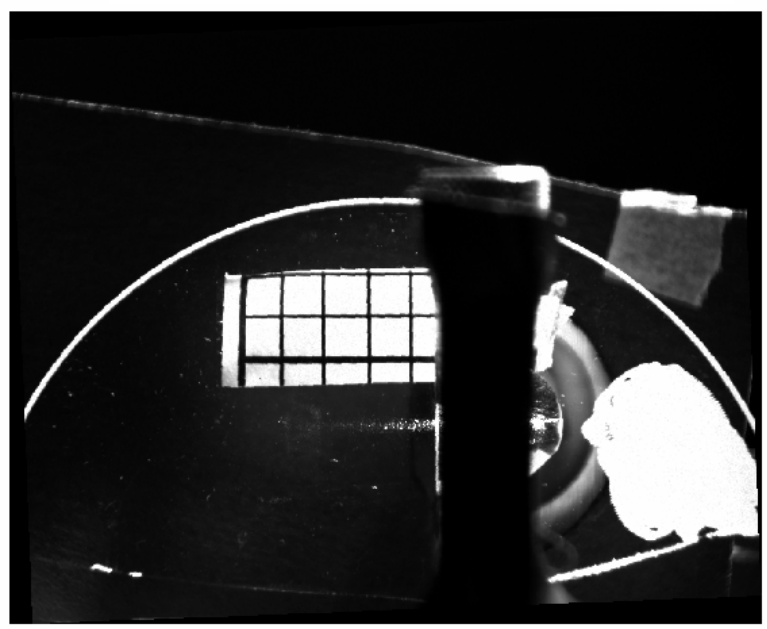

The above process is repeated for couple of times and at different regions and exposure times. The average of the losses is computed and is taken as the loss of the sample. It is possible that you would see different losses at different exposure times and that is because of the gamma factor the camera. This means that the whole area exposed is not uniformly sensitive and hence a gamma correction is needed. Usually the gamma factor is assumed to vary linearly in our working range and hence is taken to be unity.

Images as per the steps said above: 
Original Image

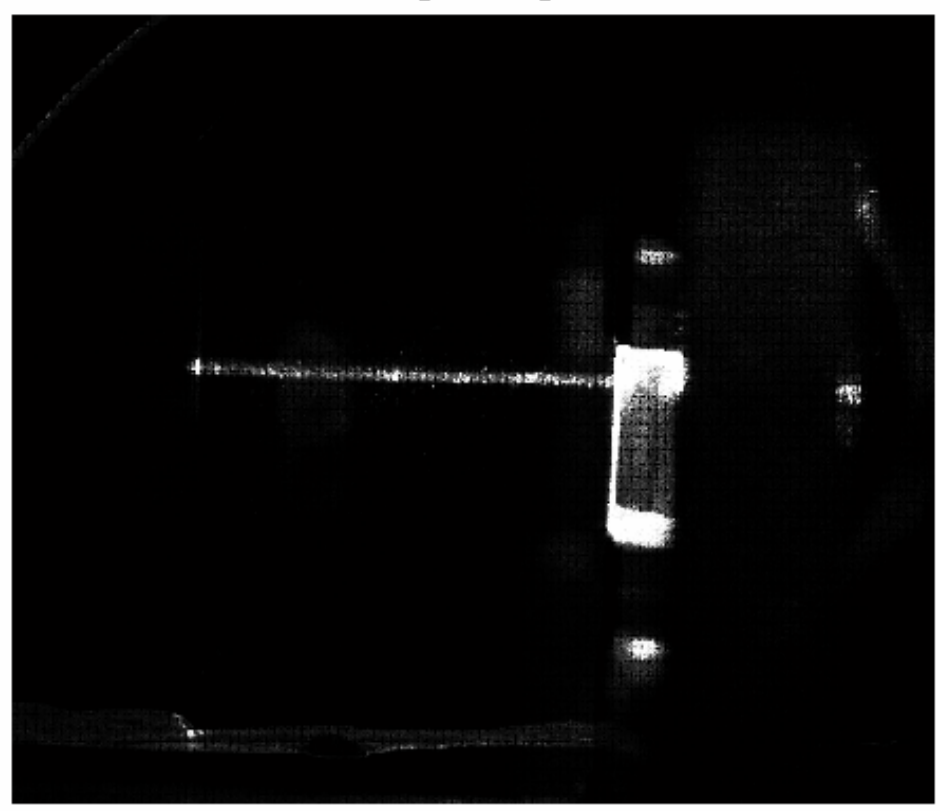

Figure 1

Original Image with area to be Cropped

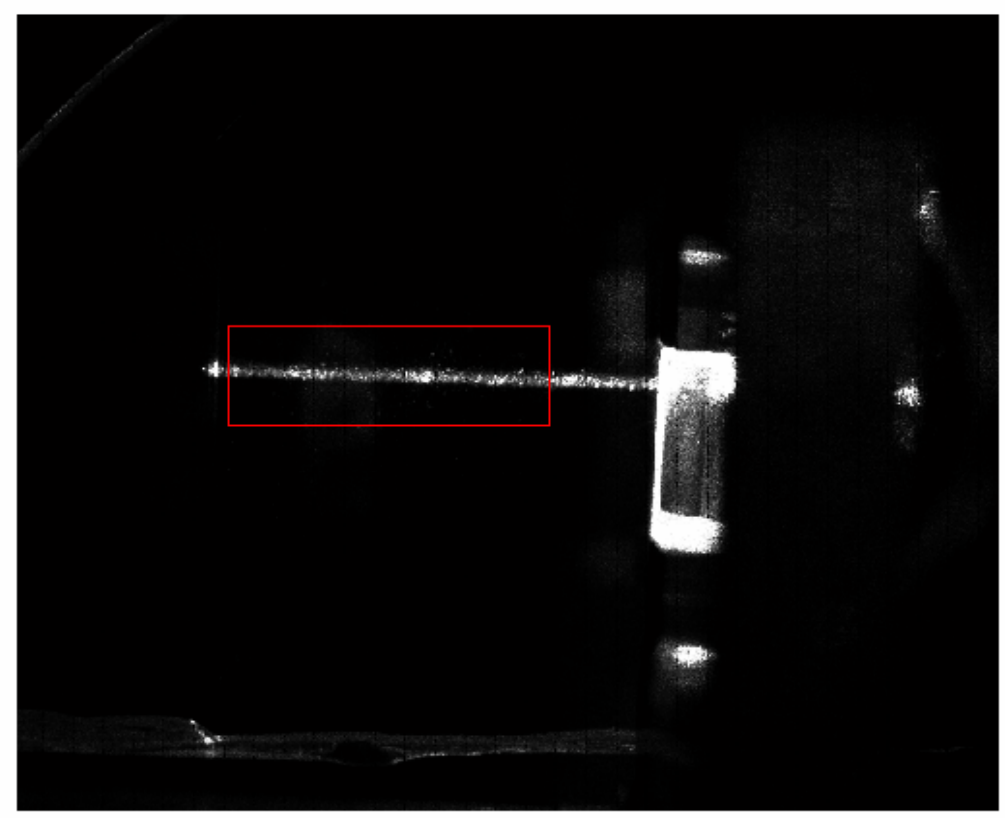

Compressed Original size image

Figure 2 
selected image

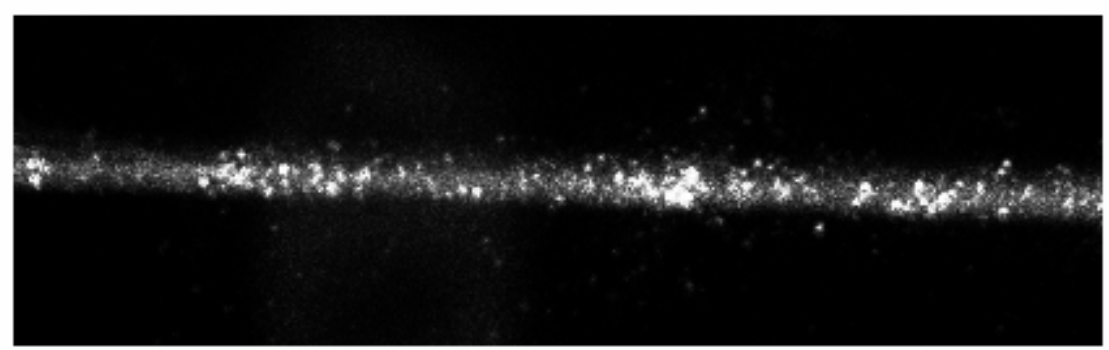

Fig 3 Chopped Area in original

Image after removing the scattering points but before blurring

Image before blurring

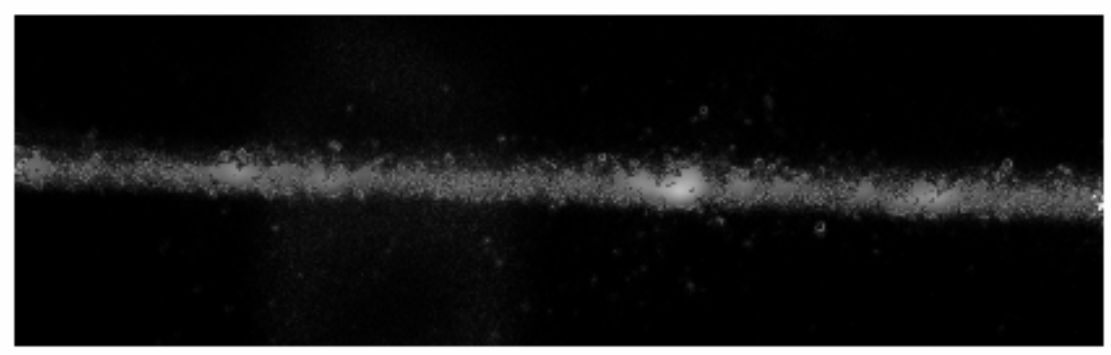

Figure 4

Image after blurring the original image (This step is optional and can be removed if the image after scattering is good. If the original image has lot of scattering points and cannot be removed from the scattering filter then this step can be used as it averages all the pixels and hence there wouldn't be any error in the measurement)

after blurring

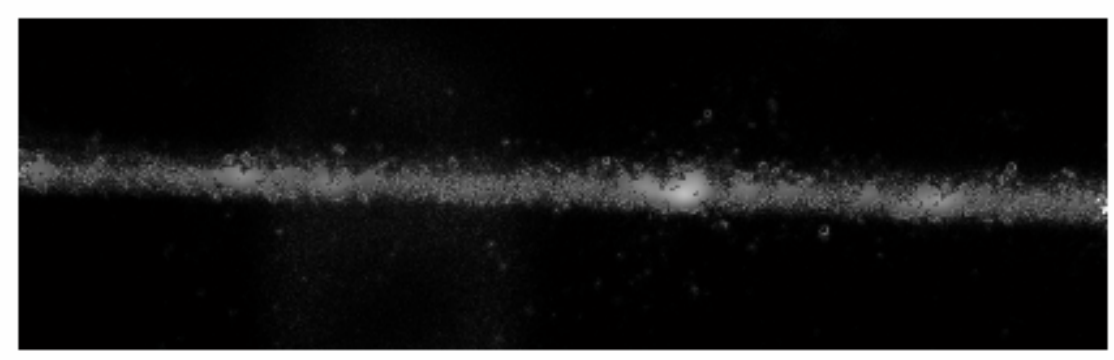

Figure 5 
This plot has the logarithm of intensities of the given image. From Figure 6 we observe that the region good for measuring losses is till 200 pixels even though there are little disturbances due to the scattering. But after 200 pixels there is enormous effect of scattering that cannot be removed by any filter and hence the user has to exclude those points using the curve fitting software.

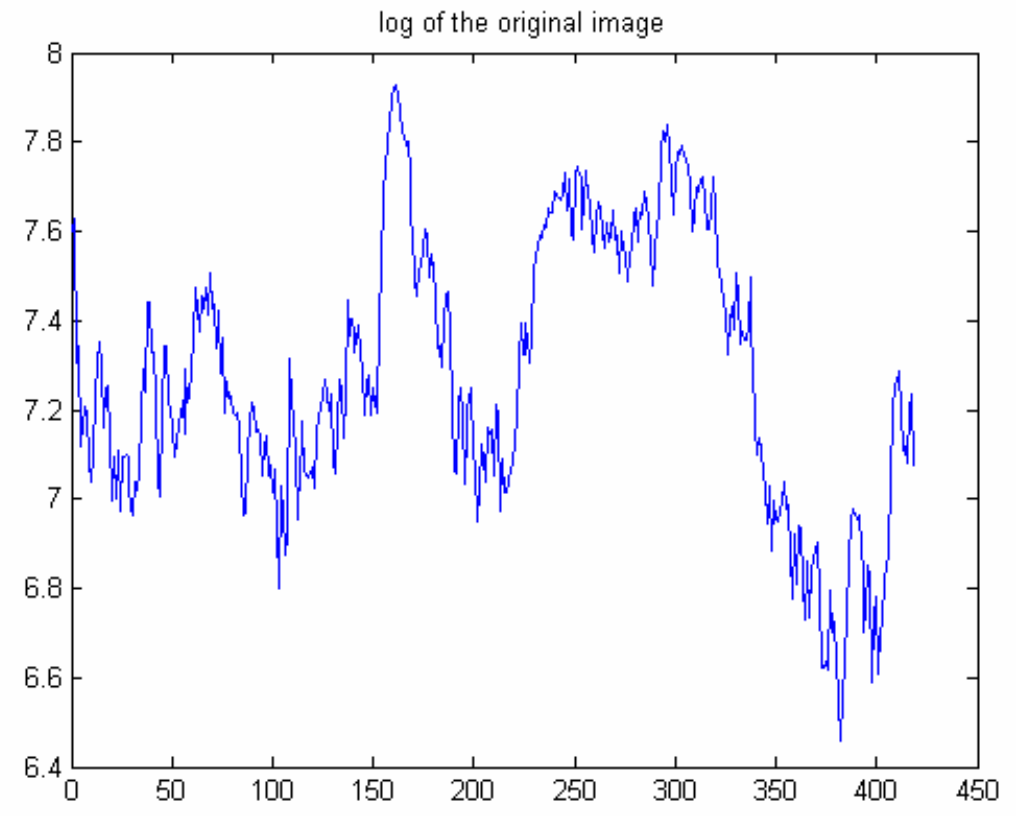

Figure 6

After excluding the unnecessary pixels the graph looks like the below figure where the shaded region corresponds to the pixels that have been neglected for loss computation.

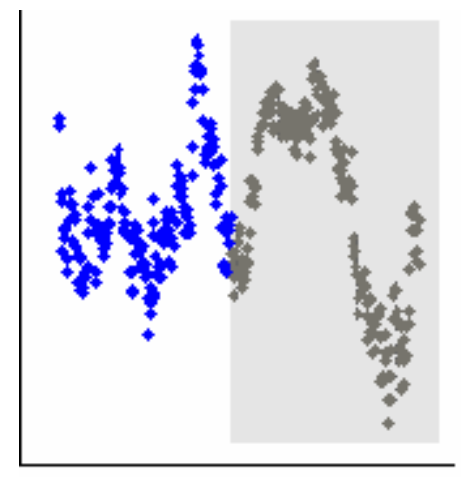

Figure 7 
The following is the image we got after employing curve fitting for the selected region. The calculation has been done for the necessary region though it cannot be seen in the image below. The slope of the line can be obtained in the fitting window. The slope of the line can be used to calculate the losses of the waveguide by using an empirical formula $4.3^{*} \alpha$ where $\alpha$ is the loss per $\mathrm{cm}$. The slope in this window gives the slope per pixel. This has to be converted to the scale of $\mathrm{cm}$ using the pixel to distance ratio. Calculation of that ratio is described in the previous section.

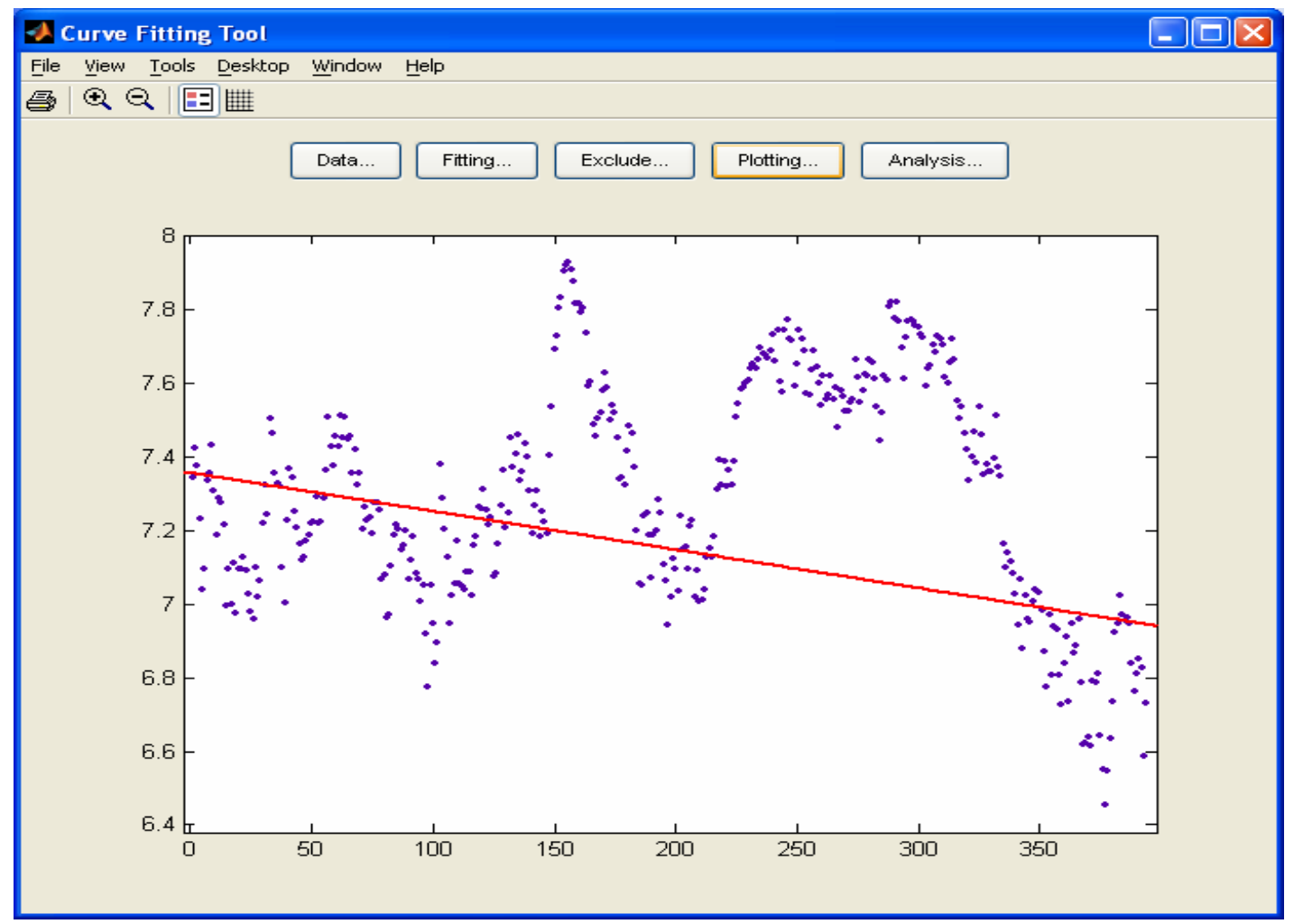

Figure 8 